University of New Hampshire

University of New Hampshire Scholars' Repository

Spring 2008

\title{
A Bayesian stormwater quality model and its application to water quality monitoring
}

Pedro M. Avellaneda

University of New Hampshire, Durham

Follow this and additional works at: https://scholars.unh.edu/dissertation

\section{Recommended Citation}

Avellaneda, Pedro M., "A Bayesian stormwater quality model and its application to water quality monitoring" (2008). Doctoral Dissertations. 438.

https://scholars.unh.edu/dissertation/438

This Dissertation is brought to you for free and open access by the Student Scholarship at University of New Hampshire Scholars' Repository. It has been accepted for inclusion in Doctoral Dissertations by an authorized administrator of University of New Hampshire Scholars' Repository. For more information, please contact Scholarly.Communication@unh.edu. 


\title{
A BAYESIAN STORMWATER QUALITY MODEL AND ITS APPLICATION TO WATER QUALITY MONITORING
}

BY

PEDRO M. AVELLANEDA

B.S., National University of Colombia, 2000

M.S., National University of Colombia, 2003

\section{DISSERTATION}

Submitted to the University of New Hampshire

In Partial Fulfillment of

the Requirements for the Degree of

\author{
Doctor of Philosophy \\ in \\ Engineering (Civil)
}

September, 2008 
UMI Number: 3333515

\section{INFORMATION TO USERS}

The quality of this reproduction is dependent upon the quality of the copy submitted. Broken or indistinct print, colored or poor quality illustrations and photographs, print bleed-through, substandard margins, and improper alignment can adversely affect reproduction.

In the unlikely event that the author did not send a complete manuscript and there are missing pages, these will be noted. Also, if unauthorized copyright material had to be removed, a note will indicate the deletion.

(B)

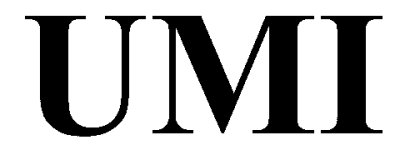

UMI Microform 3333515

Copyright 2008 by ProQuest LLC.

All rights reserved. This microform edition is protected against unauthorized copying under Title 17, United States Code.

ProQuest LLC

789 E. Eisenhower Parkway

PO Box 1346

Ann Arbor, MI 48106-1346 
This dissertation has been examined and approved.

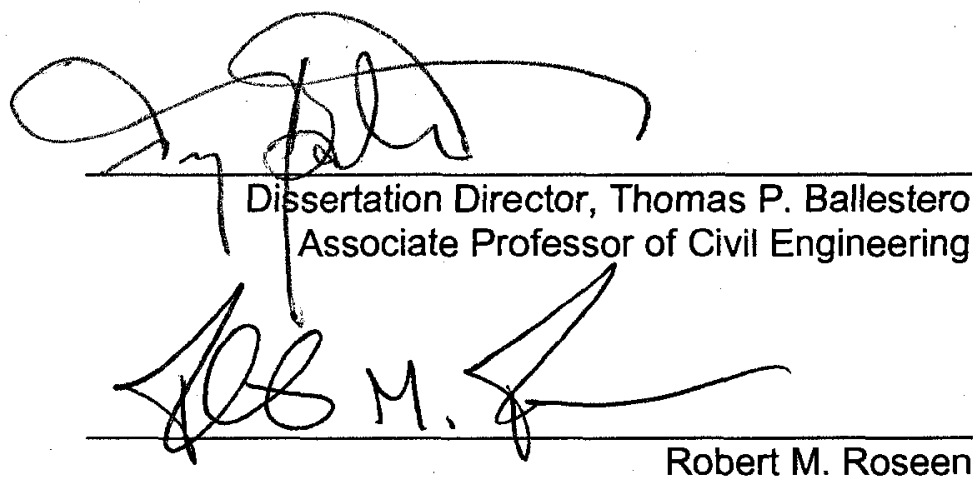

Research Assistant Professor of Civil Engineering
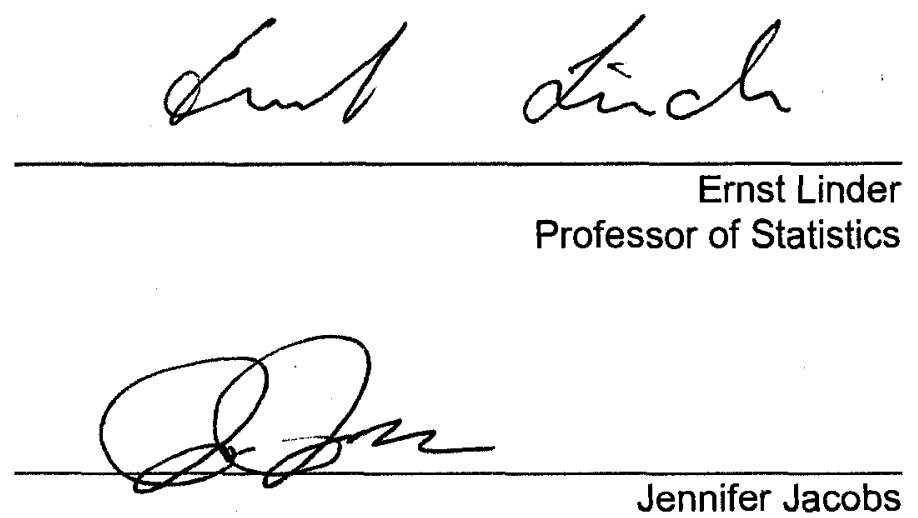

Associate Professor of Civil Engineering

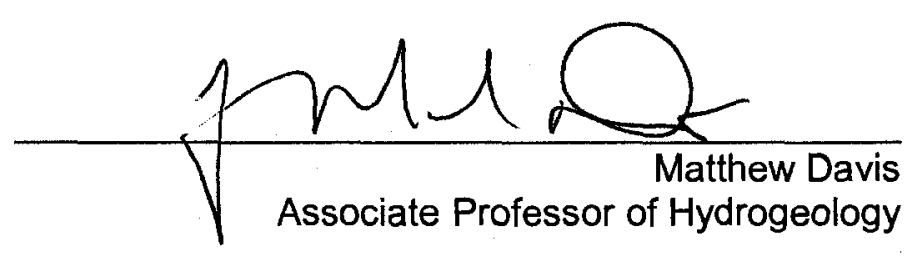

$\frac{\text { August } 2008}{\text { Date }}$ 


\section{DEDICATION}

To Estella, Pedro, Omar, Carlos, and other family members who I grew up with and taught me the importance of having a family. 


\section{ACKNOWLEDGEMENTS}

I would like to thank the members of the UNH Stormwater Center for sharing with me different academic and personal experiences since my arrival to the University of New Hampshire. Particularly, I would like to express my gratitude to my advisor and friend Tom Ballestero, who guided me during the process of obtaining my Ph.D. Tom's support was always there as well as his sense of humor and willingness to help and motivate toward accomplishing new goals. I would like to thank Rob Roseen and Jaime Houle for their patience and friendship. They were both an excellent source of information with regards to the everyday details of the project. I would like to thank the members of my doctoral committee who made positive contributions to shape the final version of this dissertation.

During my stay at the UNHSC, I worked closely with other students that became friends: Josh Briggs, Robert Wildey, Kris Houle, George Fowler, and lulia Barbu; I shared with them the feeling of being a graduate student. Their support at the site was enormous and it was always rewarding to count on them when help was needed. The friendship of the many people from the Environmental Research Group and the many others I have met in $\mathrm{NH}$ was invaluable. It was very important for me to always count on a group of friends from Colombia: Marcela Bolaños, Diana Caro, Diana Quintero, Rafael Prieto, Julian Sandoval, Nancy Palechor, Andrea Quintana, Lina Saavedra, and Gabriel. Bacca. They made me feel like I never left home. I would like to express my gratitude to Joseph for his friendship and for helping edit this document.

Lastly, I would like to thank the Cooperative Institute for Coastal and Estuarine

Environmental Technology (CICEET) and the National Oceanic and Atmospheric 
Administration (NOAA) for providing funding for this research. The UNH Stormwater

Center is housed within the Environmental Research Group (ERG) at the University of New Hampshire (UNH) in Durham, New Hampshire. 


\section{TABLE OF CONTENTS}

DEDICATION

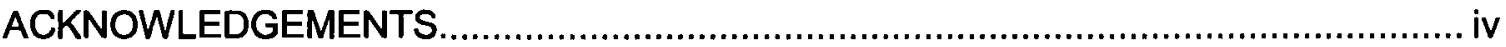

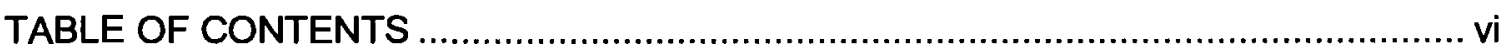

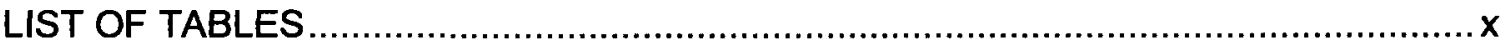

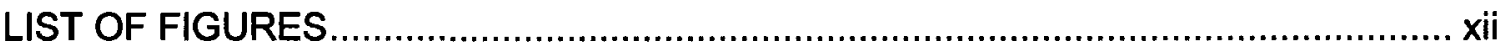

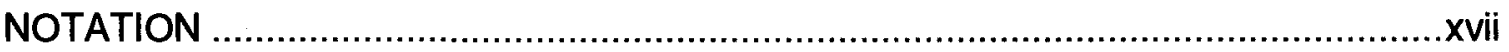

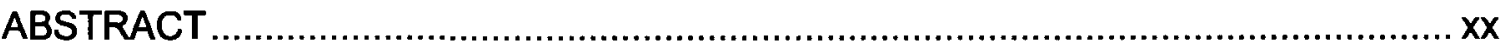

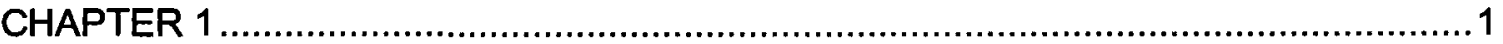

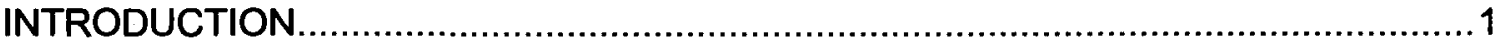

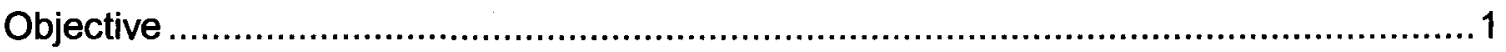

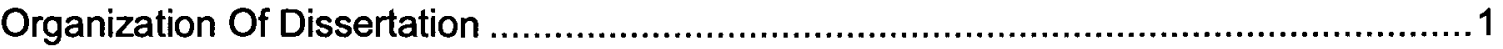

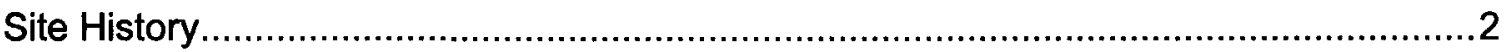

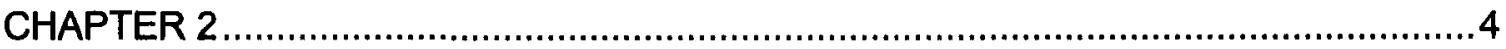

ON PARAMETER ESTIMATION OF AN URBAN STORMWATER RUNOFF MODEL ....4

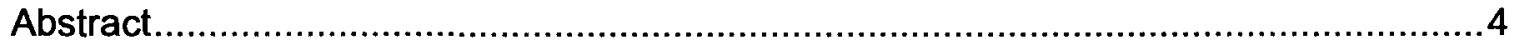

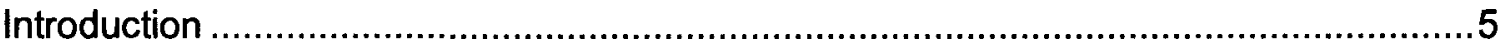

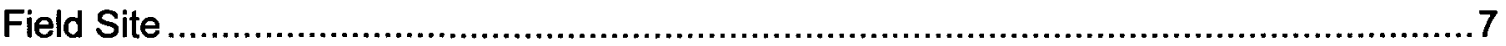

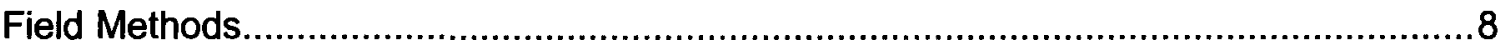

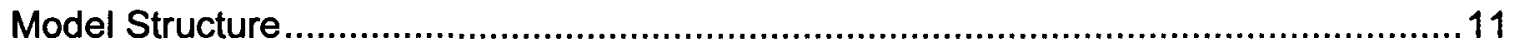

Pollutant Accumulation Model .......................................................................... 11

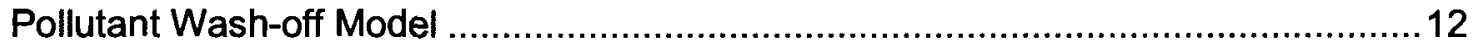

The Objective Function................................................................................. 13 


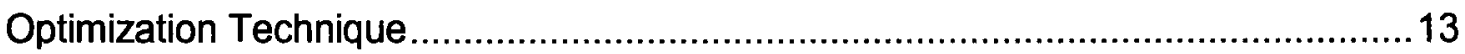

Concentration Values Below Detection Limit ......................................................16

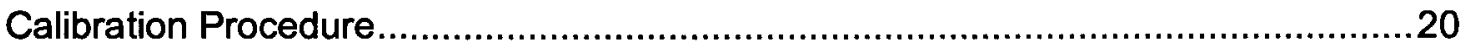

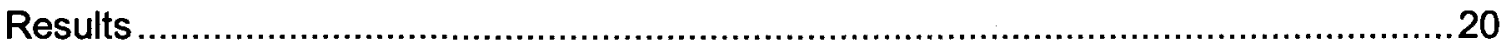

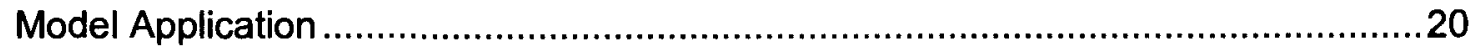

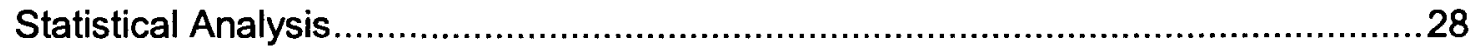

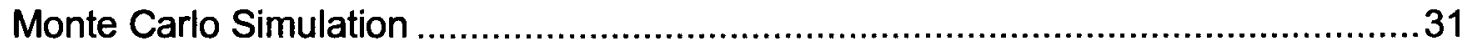

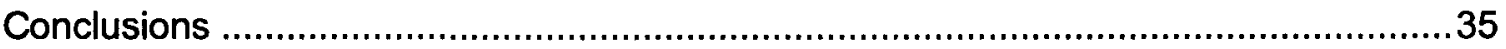

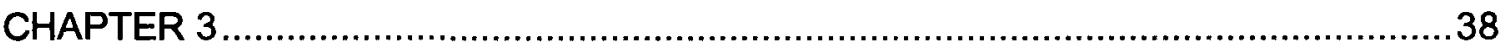

MODELING URBAN STORMWATER QUALITY TREATMENT: MODEL DEVELOPMENT AND APPLICATION TO A SURFACE SAND FILTER ......................38

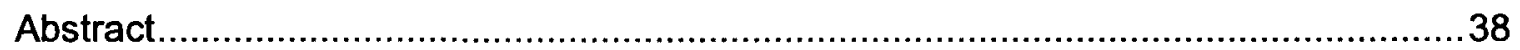

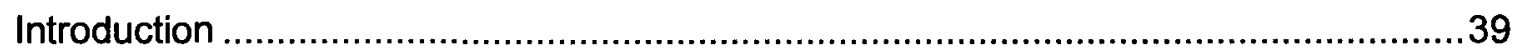

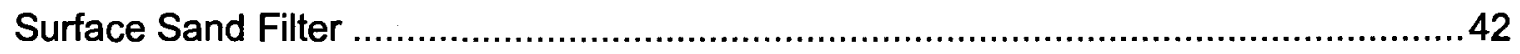

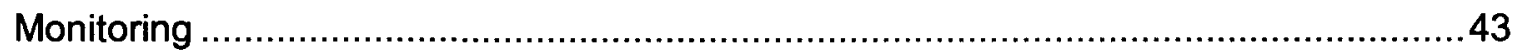

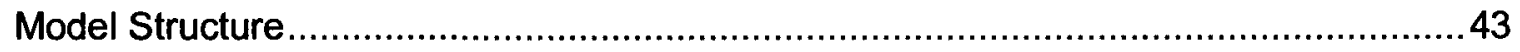

Stormwater Treatment Model ............................................................................. 43

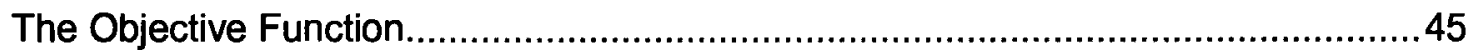

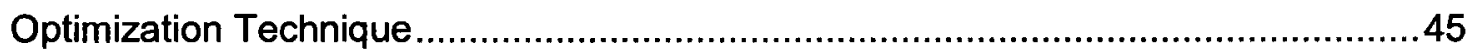

Measured Concentrations Below Detection Limit................................................46

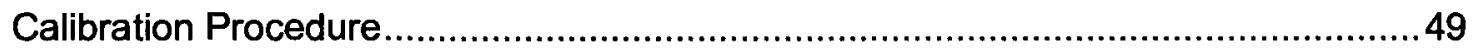

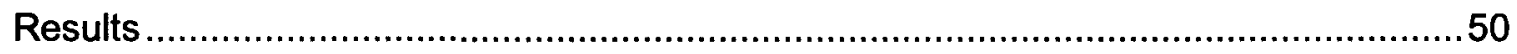

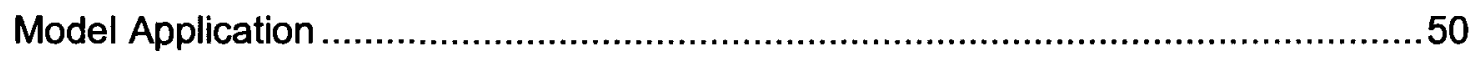

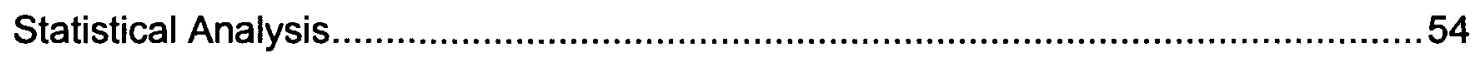

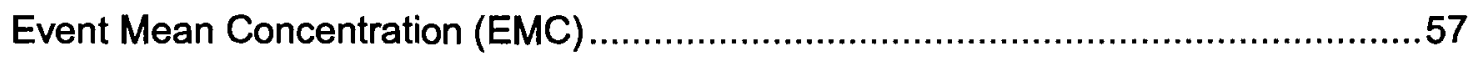

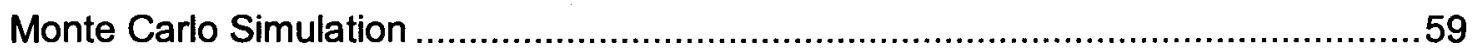




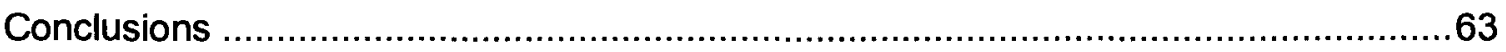

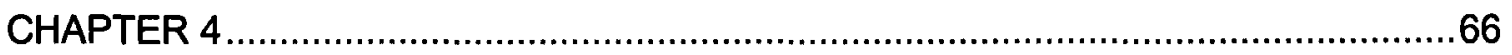

MODELING URBAN STORMWATER QUALITY TREATMENT OF A GRAVEL

WETLAND, A RETENTION POND, AND A SAND FILTER ......................................66

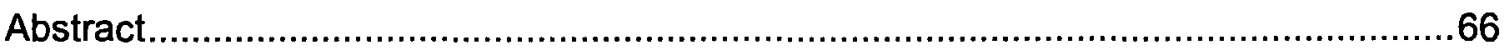

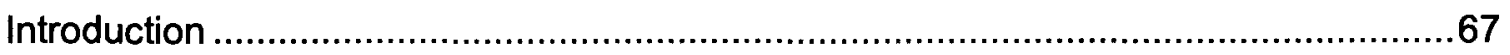

Stormwater Treatment Measures .............................................................................70

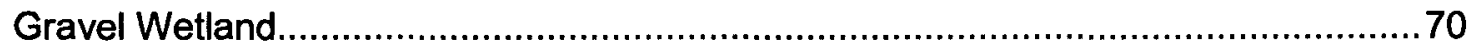

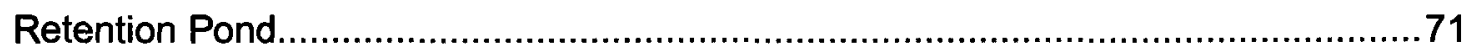

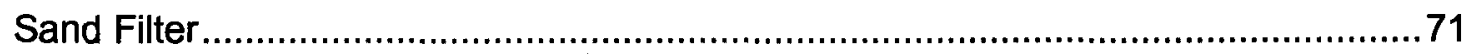

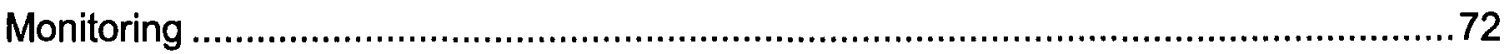

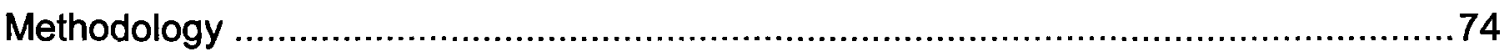

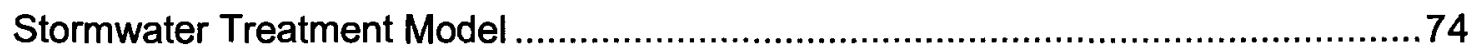

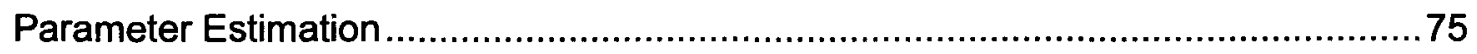

Measured Concentrations Below Detection Limit..................................................75

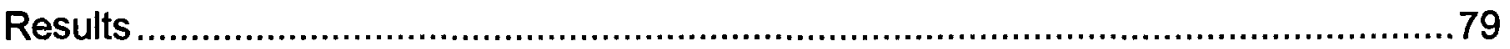

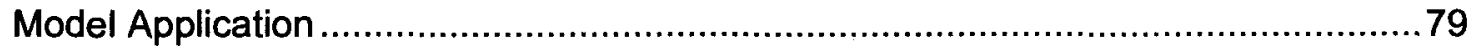

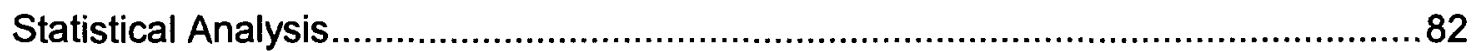

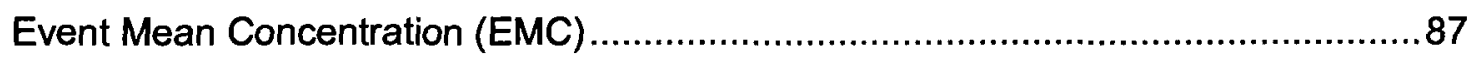

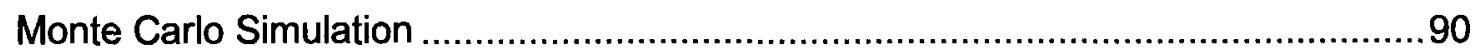

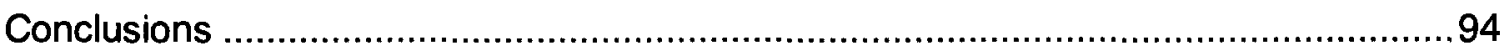

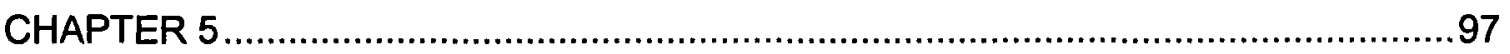

A BAYESIAN STORMWATER QUALITY MODEL AND ITS APPLICATION TO WATER

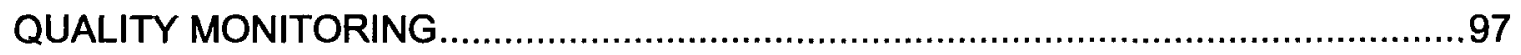

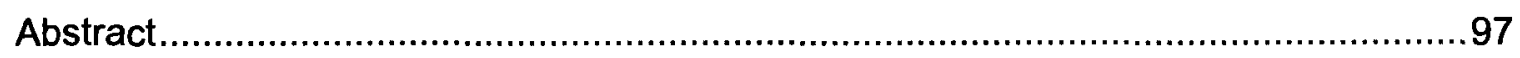

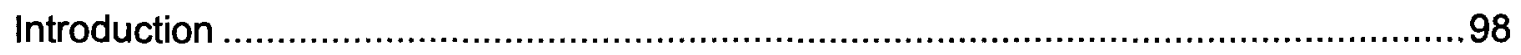




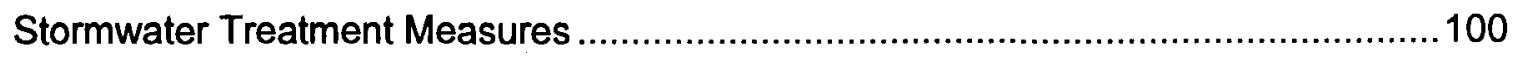

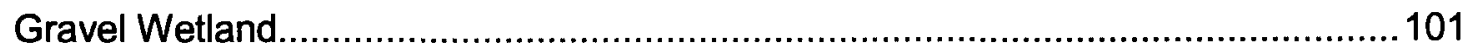

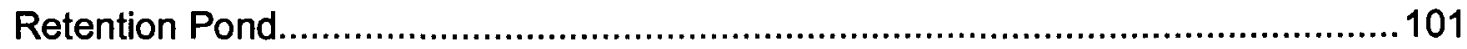

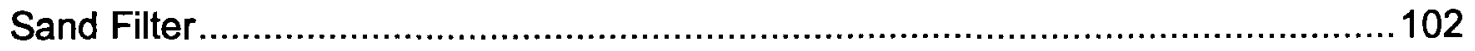

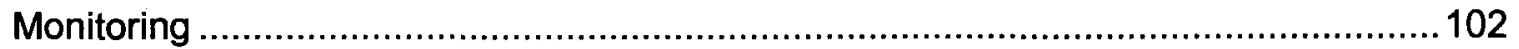

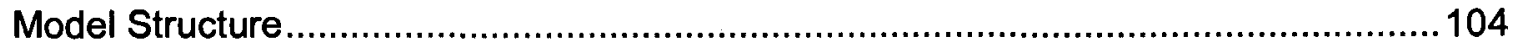

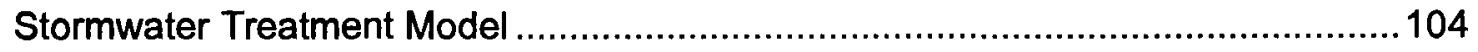

Bayesian Parameter Estimation ........................................................................ 104

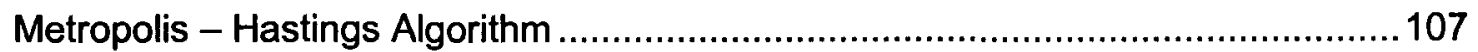

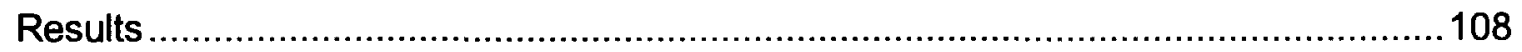

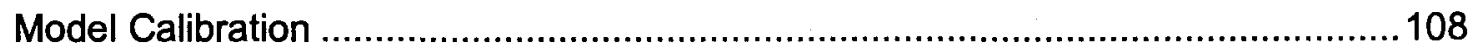

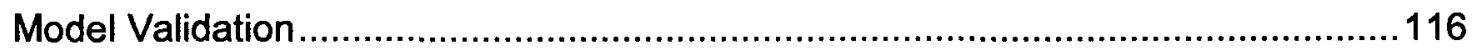

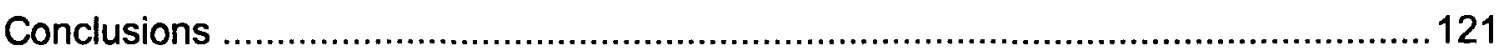

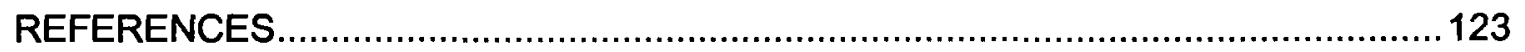

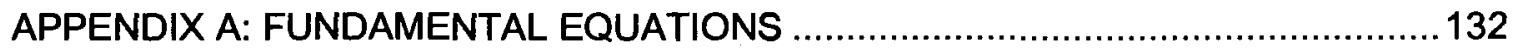




\section{LIST OF TABLES}

Table 1 Summary of monitored storm events 10

Table 2 Bounds of calibration parameters. Units: $M_{m}\left(g / m^{2}\right), k_{b}\left(d a y^{-1}\right), k_{w}\left(d a y^{w-1} / m^{3 w}\right)$, and $w(-)$. 16

Table 3 Summary of samples with concentrations reported as BDL. 17

Table 4 Parameters of the gamma distribution fitted to the data. 18

Table 5 Optimized parameter values for selected storm events. Units: $M_{m}\left(g / m^{2}\right), k_{b}$ (day $\left.{ }^{1}\right), k_{w}\left(d a y^{w-1} / m^{3 w}\right)$, and $w(-)$. 23

Table 6 Parameters of the normal distribution fitted to the optimized model parameter values. Units: $M_{m}\left(g / m^{2}\right), k_{b}\left(\right.$ day $\left.^{-1}\right), k_{w}\left(\right.$ day $\left.^{w-1} / m^{3 w}\right)$, and $w(-)$. 30

Table 7 Correlation matrixes arranged by pollutant. Units: $M_{m}\left(g / m^{2}\right), k_{b}\left(\right.$ day $\left.^{-1}\right), k_{w}\left(d a y^{w-}\right.$ $\left.1 / m^{3 w}\right)$, and $w(-)$ 31

Table 8 Observed and estimated total mass for the storms used for validation. 35

Table 9 Summary of samples with concentrations reported as BDL. 46

Table 10 Parameters of the gamma distributions fitted to the effluent data. 47

Table 11 Optimized removal rates $(k)$ and decay orders $(n)$ for different storms and contaminants 53

Table 12 Parameters of the gamma distributions fitted to removal rate optimized values.

Table 13 Frequency of optimized decay orders for the different contaminants. 56

Table 14 Observed and estimated total effluent mass for the storms used for validation. 
Table 16 Summary of effluent samples with concentrations reported as BDL 76

Table 17 Parameters of the gamma distributions fitted to the observed concentrations. 79

Table 18 Parameters of the gamma distributions fitted to optimized removal rate values.

Critical values were obtained from Miller (1956).

Table 19 Frequency of optimized decay orders for the different contaminants and systems.

Table 20 Comparison of the fitted removal rate cumulative distribution function between two systems. 85

Table 21 Observed and estimated total effluent mass for the storms used for validation.

Table 22 Summary of monitored storm events. 103

Table 23 Optimized parameter values for the 04/20/2005 storm (sand filter). 110

Table 24 Bounds of the removal rate $(k)$, which has units of $\left(\left(\mathrm{mg} / \mathrm{l}^{-\mathrm{n}+1} /\right.\right.$ day $)$.

Table 25 Summary statistics of PPDs for the removal rate (k). Gravel Wetland (GW), Sand Filter (SF), and Retention Pond (RP). S.S: Stormwater system. St: Statistic.

Table 26 Summary statistics of PPDs for the error standard deviation ( $\sigma$ ). Gravel Wetland (GW), Sand Filter (SF), and Retention Pond (RP). S.S: Stormwater system. St: Statistic. 


\section{LIST OF FIGURES}

Figure 1 Empirical and theoretical data probability distributions. C.I: Confidence Interval.

Figure 2 Results for the 03/08/2005 storm. Optimized parameter values are presented in

Table 5. Cumulative concentrations (b) were computed using estimated values. ..22

Figure 3 Results for the 04/20/2005 storm. Optimized parameter values are presented in

Table 5. Cumulative concentrations (b) were computed using estimated values. ..24

Figure 4 Results for the 01/11/2006 storm. Optimized parameter values are presented in

Table 5. Cumulative concentrations (b) were computed using estimated values. ..25

Figure 5 Optimization process and calibration results for the 03/13/2006 storm.

Optimized parameter values are shown for zinc. Optimized parameters: $M_{m}=0.13$

$\mathrm{g} / \mathrm{m}^{2}, \mathrm{k}_{\mathrm{b}}=0.0004 \mathrm{day}^{-1}, \mathrm{k}_{\mathrm{w}}=0.0002 \mathrm{day}^{\mathrm{w}-1} / \mathrm{m}^{3 \mathrm{w}}, \mathrm{w}=1.71$, and $\mathrm{b}=2.50$ days

(antecedent dry period).

Figure 6 Box and whisker plot of the optimized parameter values. Units: $M_{m}\left(g / m^{2}\right), k_{b}$

$\left(\right.$ day $\left.^{-1}\right), k_{w}\left(\right.$ day $\left.^{w-1} / m^{3 w}\right)$, and $w(-)$.

Figure 7 CDFs of optimized model parameter values (solid line) and fitted normal CDFs

(dashed line). A $95 \%$ confidence interval is reported (dotted line). Units: $M_{m}\left(\mathrm{~g} / \mathrm{m}^{2}\right)$,

$k_{b}\left(\right.$ day $\left.^{-1}\right), k_{w}\left(\right.$ day $\left.^{w-1} / m^{3 w}\right)$, and $w(-)$.

Figure 8 Monte Carlo simulations of predicted pollutant concentrations at the

catchment's outlet for the 06/21/2006 storm event. U.L: Uncertainty Limits. The dark shaded region indicates the $30 \%$ and $70 \%$ uncertainty limits. The light grey shaded region indicates the $10 \%$ and $90 \%$ uncertainty limits. Observed concentrations

values and the reported detection limits were plotted as discrete points. 
Figure 9 Monte Carlo simulations of predicted pollutant concentrations at the catchment's outlet for the 07/22/2006 storm event. U.L: Uncertainty Limits. The dark shaded region indicates the $30 \%$ and $70 \%$ uncertainty limits. The light grey shaded region indicates the $10 \%$ and $90 \%$ uncertainty limits. Observed concentrations values and the reported detection limits were plotted as discrete points. 34

Figure 10 Empirical (Data) and theoretical data (Model) probability distributions for sand filter effluent. C.I: Confidence Interval. 48

Figure 11 Results for the 03/08/2005 storm. Optimized parameter values are shown in Table 11 .51

Figure 12 Results for the 04/20/2005 storm. Optimized parameter values are shown in Table 11.

Figure 13 Box and whisker plot of optimized removal rates $(k)$ for all the contaminants and decay order models $(n=0,1,2) . k$ has units of $(m g / l)^{-n+1} / d a y$. 54

Figure 14 CDFs of optimized removal rates (Data) and fitted gamma distributions (Model). A 95\% confidence interval (C.I) is shown for each contaminant. 56

Figure 15 Sand filter CDF of Event Mean Concentrations (EMC). Out: effluent, obs: observed, and est: estimated. 58

Figure 16 Monte Carlo simulations of predicted effluent pollutant concentrations for the 05/02/2006 storm event. U.L: Uncertainty Limits. D.L: Detection Limit. The dark shaded region indicates the $30 \%$ and $70 \%$ uncertainty limits. The light grey shaded region indicates the $10 \%$ and $90 \%$ uncertainty limits. Observed concentrations values and the reported detection limits were plotted as discrete points.

Figure 17 Monte Carlo simulations of predicted effluent pollutant concentrations for the 05/09/2006 storm event. U.L: Uncertainty Limits. D.L: Detection Limit. The dark shaded region indicates the $30 \%$ and $70 \%$ uncertainty limits. The light grey shaded 
region indicates the $10 \%$ and $90 \%$ uncertainty limits. Observed concentrations values and the reported detection limits were plotted as discrete points.

Figure 18 Empirical and theoretical CDF of effluent concentrations for the gravel wetland. C.I: Confidence Interval.

Figure 19 Empirical and theoretical CDF of effluent concentrations for the retention pond. C.I: Confidence Interval. 78

Figure 20 Empirical and theoretical CDF of effluent concentrations for the sand filter. C.I:

Confidence Interval. 78

Figure 21 Box and whisker plot of the removal rate $(k)$ optimized parameter values for all the contaminants and decay order models $(n=0,1,2)$. SF: Sand Filter; RP:

Retention Pond; and GW: Gravel Wetland. The removal rate has units of $\left((\mathrm{mg} /)^{-}\right.$ $n+1 /$ day).

Figure 22 CDFs of optimized removal rates ( $\mathrm{k}$ - Data) and fitted gamma distributions (Model) for the sand filter (SF), the retention pond (RP), and the gravel wetland (GW). The removal rate has units of $\left((\mathrm{mg} / \mathrm{l})^{-\mathrm{n}+1} / \mathrm{day}\right)$. C.I: Confidence Interval........83

Figure 23 CDFs of Event Mean Concentrations (EMC) for the gravel wetland. Out:

effluent; obs: observed; and est: estimated. 88

Figure 24 CDFs of Event Mean Concentrations (EMC) for the retention pond. Out: effluent; obs: observed; and est: estimated. .89

Figure 25 CDFs of Event Mean Concentrations (EMC) for the sand filter. Out: effluent; obs: observed; and est: estimated.

Figure 26 Gravel wetland Monte Carlo simulation results for the 07/22/2006 storm. U.L: Uncertainty limits. The dark shaded region indicates the $30 \%$ and $70 \%$ uncertainty limits. The light grey shaded region indicates the $10 \%$ and $90 \%$ uncertainty limits. Observed concentrations values and the reported detection limits were plotted as discrete points. 
Figure 27 Retention pond Monte Carlo simulation results for the 06/01/2006 storm. U.L: Uncertainty limits. The dark shaded region indicates the $30 \%$ and $70 \%$ uncertainty limits. The light grey shaded region indicates the $10 \%$ and $90 \%$ uncertainty limits. Observed concentrations values and the reported detection limits were plotted as discrete points.

Figure 28 Sand filter influent information for the 04/20/2005 storm. 109

Figure 29 Parameter trace, PPDs (solid line), and prior distribution (dashed line) for the Gravel Wetland (TPH-D) and different decay orders (n). The removal rate (k) has units of $\left(\mathrm{mg} / \mathrm{l}^{-\mathrm{n}+1} /\right.$ day and the error standard deviation $(\sigma)$ units of $\mathrm{mg} / \mathrm{l}$. The acceptance rate $\left(a_{r}\right)$ is reported.

Figure 30 Parameter trace, PPDs (solid line), and prior distribution (dashed line) for the Sand Filter (TPH-D) and different decay orders $(n)$. The removal rate (k) has units of $\left(\mathrm{mg} / \mathrm{l}^{-\mathrm{n}+1} /\right.$ day and the error standard deviation $(\sigma)$ units of $\mathrm{mg} / \mathrm{l}$. The acceptance rate $\left(a_{r}\right)$ is reported.

Figure 31 Parameter trace, PPDs (solid line), and prior distribution (dashed line) for the Sand Filter (DIN) and different decay orders $(n)$. The removal rate (k) has units of $(\mathrm{mg} / \mathrm{l})^{-n+1} /$ day and the error standard deviation $(\sigma)$ units of $\mathrm{mg} / \mathrm{l}$. The acceptance rate $\left(a_{r}\right)$ is reported.

Figure 32 Monte Carlo simulations of predicted sand filter effluent pollutant concentrations for the 05/02/2006 storm event. U.L: Uncertainty limits. D.L: Detection limit. The dark shaded region indicates the $30 \%$ and $70 \%$ uncertainty limits. The light grey shaded region indicates the $10 \%$ and $90 \%$ uncertainty limits. Observed concentrations values and the reported detection limits were plotted as discrete points. 
Figure 33 Monte Carlo simulations of predicted gravel wetland effluent pollutant concentrations for the 07/22/2006 storm. U.L: Uncertainty limits. D.L: Detection limit. The dark shaded region indicates the $30 \%$ and $70 \%$ uncertainty limits. The light grey shaded region indicates the $10 \%$ and $90 \%$ uncertainty limits. Observed concentrations values and the reported detection limits were plotted as discrete points.

Figure 34 Monte Carlo simulations of predicted retention pond effluent pollutant concentrations for the 06/01/2006 storm. U.L: Uncertainty limits. D.L: Detection limit. The dark shaded region indicates the $30 \%$ and $70 \%$ uncertainty limits. The light grey shaded region indicates the $10 \%$ and $90 \%$ uncertainty limits. Observed concentrations values and the reported detection limits were plotted as discrete points. 


\section{NOTATION}

a: $\quad$ Reduction coefficient (simulating annealing algorithm).

$\mathrm{a}_{\mathrm{r}}$ : Acceptance rate (Metropolis - Hastings algorithm).

b: $\quad$ Antecedent dry period (days).

$\mathrm{C}_{\text {est }}: \quad$ Estimated concentrations $(\mathrm{mg} / \mathrm{l})$ when using $\Theta$.

$\mathrm{C}_{\mathrm{in}}: \quad$ Influent concentration $(\mathrm{mg} / \mathrm{l})$.

$\mathrm{C}_{0}$ : Initial concentration within the system $(\mathrm{mg} / \mathrm{l})$.

$\mathrm{C}_{\mathrm{obs}}: \quad$ Observed concentrations (mg/l).

$\mathrm{C}_{\text {out: }} \quad$ Effluent concentration $(\mathrm{mg} / \mathrm{l})$.

$\mathrm{C}_{\text {out,obs: }}$ Effluent observed concentrations (mg/l).

Cout,est: $\quad$ Effluent estimated concentrations (mg/l).

$C_{\text {out }, \text { st }}^{k}$ : Estimated effluent concentration using only the removal rate parameter (mg/l).

$C_{\text {out }, \text { sst }}^{k, \sigma^{2}}: \quad$ Estimated effluent concentration using both the removal rate and the error variance $(\mathrm{mg} / \mathrm{l})$.

dS/dt: Change in storage $\left(\mathrm{m}^{3} /\right.$ day $)$.

D: $\quad$ Parameter of the Kolmogorov-Smirnov test.

$D_{\text {critical }} \quad$ Critical value of the $D$ parameter of the Kolmogorov-Smirnov test.

$D_{10}: \quad 10 \%$ of the particles are smaller than $D_{10}(\mathrm{~mm})$.

$D_{50}: \quad 50 \%$ of the particles are smaller than $D_{50}(\mathrm{~mm})$.

$D_{85:} \quad 85 \%$ of the particles are smaller than $D_{85}(\mathrm{~mm})$.

DIN: $\quad$ Comprised of nitrate, nitrite, and ammonia. 


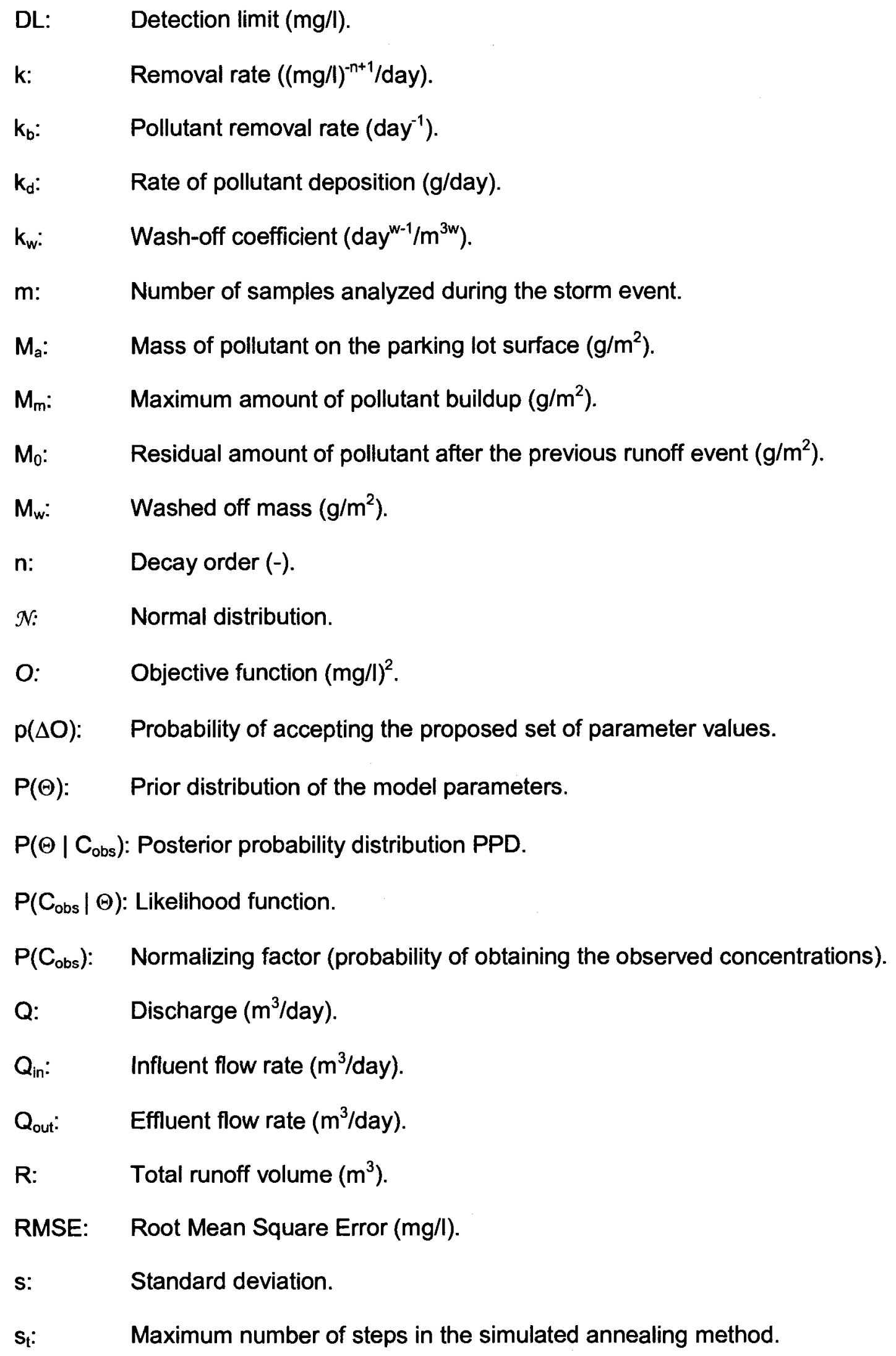


S: $\quad$ Storage within the system $\left(\mathrm{m}^{3}\right)$.

SSE: $\quad$ Sum of squared errors.

t: $\quad$ Time (days).

T: $\quad$ Temperature (simulated annealing).

TPH-D: Total petroleum hydrocarbons - diesel range hydrocarbons.

TSS: $\quad$ Total suspended solids.

w: Wash-off exponent (-).

WQV: $\quad$ Water quality volume $\left(\mathrm{m}^{3}\right)$.

x: Input variables such as: influent fiow and concentrations, effluent flow, storage, and change in storage.

$\bar{x}: \quad$ Mean.

Zn: Zinc.

$\alpha$ : Level of significance.

$\alpha_{\text {ratio: }} \quad$ Likelihood ratio.

$\alpha_{i . g}: \quad$ Parameter of the inverse gamma probability function.

$\beta_{\mathrm{i} . \mathrm{g}}$ : $\quad$ Parameter of the inverse gamma probability function.

$\Delta \mathrm{t}: \quad$ Time interval $(\mathrm{min})$.

$\Theta: \quad \quad$ Parameter values $(k, n)$.

$\Theta^{\mathrm{t}}: \quad$ Current state of the parameters.

$\Theta: \quad$ Parameters generated from a proposal distribution.

$\Delta \mathrm{O}: \quad$ Change in the objective function $\left(\mathrm{mg} / \mathrm{l}^{2}\right.$.

$\gamma: \quad$ Shape parameter of the gamma distribution.

$\theta: \quad$ Scale parameter of the gamma distribution.

६: $\quad$ Residual.

$\sigma^{2}: \quad$ Error variance. 


\begin{abstract}
A BAYESIAN STORMWATER QUALITY MODEL AND ITS APPLICATION TO WATER QUALITY MONITORING
\end{abstract}

by

Pedro Avellaneda

University of New Hampshire, September, 2008

Stormwater runoff is a topic of research that over the years has increasingly grown due to its impact on our water resources. Treatment systems have been developed to mitigate this impact by preserving the pre-development hydrologic and water quality characteristics of the drainage areas. Understanding of the systems' treatment capabilities is required for stormwater management. The goal of this research was to study the application of a decay treatment model as a conceptual tool for understanding the pollutant removal characteristics of stormwater systems. Three systems were studied in this research: a sand filter, a gravel wetland, and a retention pond. The contaminants under consideration include: total suspended solids (TSS), total petroleum hydrocarbons - diesel range hydrocarbons (TPH-D), dissolved inorganic nitrogen (DIN, comprised of nitrate, nitrite, and ammonia), and zinc ( $\mathrm{Zn})$.

The mathematical model was based on the mass balance principle and the assumption that an n-order decay model describes the complex processes of pollutant removal (for example sedimentation, biodegradation, filtration, plant uptake, and 
chemical precipitation). The model was defined by the parameters of removal rate (k) and the decay order (n). For each treatment system, a collection of storm events was monitored between 2004 and 2006. Monitoring of the treatment systems was performed in a side by side fashion so that each system received the same stormwater quantity and quality. This configuration made possible a comparison of the calibrated parameters obtained for each system. The best set of parameters of the decay model was determined by using a simulated annealing technique as part of the optimization process. Monte Carlo simulations were performed to describe the uncertainty of the estimated effluent concentrations. The gravel wetland achieved the highest median DIN and TSS removal rates. For TPH-D, the highest median removal rates were achieved by the retention pond and gravel wetland. The sand filter and the gravel wetland achieved the highest median $\mathrm{Zn}$ removal rates. First and second order decay models were more likely to describe the observed effluent concentrations.

A Bayesian statistical approach for determining parameter uncertainty of the stormwater treatment model is presented. For this model, it was found that a second order decay model was more likely to reproduce estimated effluent concentrations. Mean removal rate values were computed from the posterior distributions. Specifically, for the gravel wetland: $k_{T S S}=59, k_{Z n}=2115, k_{T P H-D}=88, k_{D I N}=7$; for the sand filter: $k_{T S S}=$ $1.7, \mathrm{k}_{\mathrm{Zn}}=1568, \mathrm{k}_{\mathrm{TPH}-\mathrm{D}}=57, \mathrm{k}_{\mathrm{DIN}}=2 ;$ and for the retention pond: $\mathrm{k}_{\mathrm{TSS}}=0.8, \mathrm{k}_{\mathrm{Zn}}=4645$, $k_{T P H-D}=68, k_{D I N}=8\left(k\right.$ in units of $(m g / l)^{-1} /$ day $)$. 


\title{
CHAPTER 1
}

\section{INTRODUCTION}

\begin{abstract}
Objective
Stormwater runoff is a topic of investigation that over the years has increasingly grown due to its impact on our water resources. Treatment systems have been developed to mitigate this impact by preserving the pre-development hydrologic and water quality characteristics of the drainage areas. Understanding of the systems' treatment capabilities is required for stormwater management. The goal of this research is to investigate the application of a decay treatment model as a conceptual tool for understanding the pollutant removal characteristics of stormwater systems. Three systems were studied in this research: a sand filter, a gravel wetland, and a retention pond. Contributions to the calibration and validation of the treatment model are provided.
\end{abstract}

\section{Organization Of Dissertation}

This dissertation consists of five chapters, four of them written as self-contained individual papers for submission to peer-reviewed journals. An introductory chapter presents the objectives and organization of dissertation.

Chapter 2, "On parameter estimation of an urban stormwater runoff model", describes the application of an accumulation and wash-off model for estimating stormwater quality 
from an urban watershed. Optimized parameter values are provided for a collection of contaminants.

Chapter 3, "Modeling urban stormwater quality treatment: Model development and application to a surface sand filter", describes a decay treatment model for stormwater management. A probabilistic approach for estimating effluent water quality from a sand filter is introduced.

Chapter 4 is "Modeling urban stormwater quality treatment of a gravel wetland, a retention pond, and a sand filter". The approach described in Chapter 3 is extended to a gravel wetland and a retention pond. A comparison of the model performance among the three systems is then provided.

Chapter 5, “A Bayesian stormwater quality model and its application to water quality monitoring", describes how a Bayesian statistical approach is applied to the model developed in Chapters 3 and 4 for describing the variability of the model parameters.

\section{Site History}

The field site for this research is located at the University of New Hampshire Stormwater Center (UNHSC), Durham, NH. Since 2003, the center has conducted research to evaluate and enhance the performance of stormwater management systems. Stormwater runoff is provided by a $36,000 \mathrm{~m}^{2}$ commuter parking lot that is used to near capacity during the school year by a combination of passenger vehicles and bus traffic. Pollutant concentrations are above, or equal to, national norms for commercial parking lot runoff. The climatology of the area is characterized as coastal, with an average annual precipitation of $1220 \mathrm{~mm}$ uniformly distributed throughout the year. The average annual snowfall is $2032 \mathrm{~mm}$. 
The field site contains three classes of stormwater treatment systems: conventional, structural best management practices (BMPs) such as swales and retention ponds; low impact development (LID) stormwater designs such as tree filters, bioretention systems, and a gravel wetland; and manufactured BMPs such as hydrodynamic separators and subsurface infiltration / filtration systems. The parallel configuration of the systems normalizes the stormwater contaminant loading (same influent for each system), allowing for a comparison of performance. Each system is uniformly sized to address a Water Quality Volume (WQV) of $92.5 \mathrm{~m}^{3}$. 


\title{
CHAPTER 2
}

\section{ON PARAMETER ESTIMATION OF AN URBAN STORMWATER RUNOFF MODEL}

\begin{abstract}
An accumulation and wash-off model was applied and calibrated on an asphalt parking lot located in the northeastern United States. The field measured data consisted of rainfall, flow, and runoff samples taken over 26 storm events monitored from 2004 to 2006. The contaminants under consideration include: total suspended solids (TSS), total petroleum hydrocarbons - diesel range hydrocarbons (TPH-D), dissolved inorganic nitrogen (DIN, comprised of nitrate, nitrite, and ammonia), and zinc ( $\mathrm{Zn}$ ). The aim of the study was to find the best fitting parameter values on a storm by storm basis. Subsequently, the range and variability of these parameters are provided for modeling purposes and other urban stormwater quality applications. A normal distribution was fitted to the optimized model parameter values to describe their distributions. A simulated annealing algorithm was used for the parameter optimization technique. Several examples are given in order to illustrate the methodology and the performance of the model. Finally, a Monte Carlo simulation was performed to assess the capability of the model to predict contaminant concentrations at the watershed's outlet.
\end{abstract}




\section{Introduction}

As a consequence of the awareness of the pollution impact due to stormwater, managers need tools to evaluate and control stormwater according to water quality criteria (House et al., 1993; Ahyerre et al., 1998). Application of storm water runoff quality models is necessary to improve: watershed management, selection of stormwater technologies, parameterization for modeling purposes, understanding of model limitations, and assessment of how much confidence one could have in these models.

Stormwater contaminant runoff models are commonly used for urban stormwater quality applications (DeCoursey et al., 1985; Tsihrintzis et al., 1997; Zoppou, 2001). These models are usually a combination of accumulation and wash-off equations. The accumulation of contaminants on impervious surfaces is non-linear and follows an exponential increase as it approaches to a maximum value (Alley and Smith, 1991). The total amount of contaminants is a function of the initial mass on the surface area and the length of the antecedent storm dry period. The common accumulation model: is entirely deterministic; follows an exponential time history; and does not take into account the spatial distribution of the contaminants (Alley and Smith, 1991).

Sartor et al. (1974) performed several field experiments on street surfaces when investigating a mathematical expression for simulating the wash-off of contaminants. The study revealed that an exponential decay model was able to reproduce measured observations. This exponential decay model was a function of the available mass and rainfall intensity. Other studies have proposed the usage of the total runoff volume or runoff rate as opposed to rainfall rate (Haiping and Yamada, 1996). Computer models normally use a runoff rate approach and include a wash-off coefficient in the erosion model (Rossman, 2004). 
There are a variety of techniques available for model calibration purposes. The simplest method is trial and error. Genetic algorithms, gradient-based functions, and simulated annealing are just a few of the more advanced calibration procedures that have been proposed to perform the optimization process (Hopgood, 2001). All these methods stochastically explore the domain of the objective function by using a different goodness-of-fit criterion.

Alley and Smith (1981) provided an understanding of model sensitivity to the parameters for an urban runoff quality model. Water quality constituents in their study included total nitrogen, total lead, and suspended solids. To assess parameter sensitivity, mathematical expressions were derived for each model parameter by direct differentiation of the analytical accumulation and wash-off equations. The wash-off coefficient was found to be the parameter with more variability among the parameters of the accumulation and wash-off model. Further analysis of the variability of the parameters was recommended.

Haiping and Yamada (1996) employed an adaptive step-size, random search algorithm to calibrate an urban runoff model. The wash-off model used in their study was a function of rainfall intensity. However, a wash-off exponent was not considered. The contaminants under consideration were total nitrogen, total solids, and total phosphorus. The model was applied continuously (long-term simulation) over a four month simulation period. Bounds of the calibration parameters were provided.

Other studies (Kanso et al., 2003; Chen and Adams, 2006) analyzed the performance of a similar model that incorporates a wash-off exponent as a new parameter (the exponent of $R$ in Equation (2)). This approach is recommended when the watershed seems to have a high non-linear response to rainfall. Recent computer models include a wash-off exponent to improve the quality of the results (Rossman, 2004). 
Gaume et al. (1998) focused their study on the estimation and interpretation of parameter uncertainties. A wash-off exponent was used on the erosion model (Equation (2)). Difficulties during the calibration process were reported, mostly due to large uncertainty in the parameter values. A lack of recorded data was thought to be the main reason for calibration failure. This study provided calibrated parameter values for suspended solids on a storm by storm basis over the course of five rainfall events.

For the study herein, stormwater runoff flow and water quality were monitored at a parking lot in Durham, $\mathrm{NH}$. These data were collected to calibrate an accumulation and wash-off model. This study does not intend to evaluate different stormwater models. Instead, a model was selected for calibration and results are provided for a range of contaminants that have either not been studied or have little documentation. The selected model is similar to the one used by Haiping and Yamada (1996) (Equation (1) and (2)) and includes a wash-off exponent. This study focuses on the calibration of this model for a group of four contaminants using 26 storm events; identifying appropriate probability distributions for each parameter of the model and testing the prediction of pollutant concentrations at the catchment's outlet.

\section{Field Site}

The study area is a $36,000 \mathrm{~m}^{2}$ commuter parking lot at the University of New Hampshire in Durham, NH. The parking lot is curbed, constructed of standard dense mix impervious asphalt, and drained by catch basins. Parking lot usage is a combination of passenger vehicles and routine bus traffic. A total of 786 parking spaces are used to near full capacity throughout the school year. During the summer, the parking lot receives much less use than during the regular school year. Additionally, during the 
summer the bus service is suspended. The parking lot is subject to plowing, salting (approximately 19 tons/year), and sanding ( $<5 \%$ of the mix) during the winter.

Stormwater runoff flows from catch basins into a central 914-mm diameter reinforced concrete pipe. The runoff sampling station is located at the outlet of this pipe. The runoff time of concentration for the lot is 22 minutes, with slopes ranging from $1.5-2.5 \%$. Contaminant concentrations are similar to typical values reported in stormwater (Pitt et al., 1995; Zoppou, 2001; Minton, 2002). The climatology of the area is characterized as coastal, with an average annual precipitation of $1220 \mathrm{~mm}$ uniformly distributed throughout the year.

\section{Field Methods}

A total of 26 discrete rainfall events were monitored between August 2004 and September 2006. Water sampling was performed at the outlet of the 914-mm pipe using a 6712 SR ISCO automated sampler provided with a stainless steel strainer, $9.52 \mathrm{~mm}$ vinyl collection tubing, and 24 discrete 1 -liter polypropylene bottles and maintained at $4^{\circ} \mathrm{C}$. A storm was sampled if the total precipitation was higher than $2.5 \mathrm{~mm}$ and preceded by at least 72 hours of dry weather. An ISCO Model 674 tipping bucket rain gauge was used to monitor rainfall. The minimum depth that the rain gauge could record was $0.254 \mathrm{~mm}$.

Flow was monitored at the outlet of the $914-\mathrm{mm}$ pipe, so a hydraulic model to estimate discharge was not necessary. The sampler was triggered on the basis of preset flow conditions (influent flow $>82 \mathrm{~m}^{3} /$ day). 24 samples were taken for each storm event for a 24 hour collection period; however, normally only 8-12 of these samples were sent to the laboratory to be analyzed. Samples were analyzed with the intent of linearizing the

runoff concentration graph. The sampling program was designed to collect five samples 
within the first flush ( 4 min interval) and spread out the remaining samples over the rest of the hydrograph (24 min interval). ISCO $1 \mathrm{~L}$ ProPak disposable sampling bags were used to collect the stormwater samples.

Runoff constituent analysis included: total suspended solids (TSS), total petroleum hydrocarbons - diesel range hydrocarbons (TPH-D), dissolved inorganic nitrogen (DIN, comprised of nitrate, nitrite, and ammonia), and zinc ( $\mathrm{Zn})$. Stormwater samples were analyzed by a laboratory that is state-certified for drinking water and wastewater. Guidance documents on collecting environmental data and the site quality assurance project plan were followed (EPA, 2006). Analyses were performed using techniques according to standard methods (APHA et al., 2005). Additional information on watershed characteristics and the sample monitoring program is found in Roseen et al. (2006).

A summary of the monitored storm events is shown in Table 1. This data set includes variations of storm duration, peak flow, total volume, antecedent dry period, and season. Table 1 shows a variety of storm event characteristics, which is important when characterizing water quality of stormwater. 
Table 1 Summary of monitored storm events

\begin{tabular}{|c|c|c|c|c|c|c|c|}
\hline $\begin{array}{l}\text { Rainfall } \\
\text { Event } \\
(\mathrm{m} / \mathrm{d} / \mathrm{y})\end{array}$ & $\begin{array}{c}\text { Peak } \\
\text { Intensity } \\
(\mathrm{mm} / \mathrm{hr})\end{array}$ & $\begin{array}{l}\text { Rain } \\
\text { Duration } \\
\text { (min) }\end{array}$ & $\begin{array}{l}\text { Total } \\
\text { Depth } \\
(\mathrm{mm})\end{array}$ & $\begin{array}{c}\text { Peak } \\
\text { Flow } \\
\left(\mathrm{m}^{3} / \text { day }\right)\end{array}$ & $\begin{array}{l}\text { Volume } \\
\qquad\left(\mathrm{m}^{3}\right)\end{array}$ & $\begin{array}{c}\text { Anticedent } \\
\text { Dry Period } \\
\text { (days) }\end{array}$ & Season \\
\hline $9 / 18 / 2004$ & 15 & 1075 & 50 & 5642 & 1364 & 7.0 & Fall \\
\hline $10 / 30 / 2004$ & 21 & 705 & 11 & 8678 & 281 & 13.0 & Fall \\
\hline $11 / 24 / 2004$ & 9 & 705 & 18 & 4394 & 530 & 3.5 & Fall \\
\hline $1 / 14 / 2005$ & 24 & 645 & 17 & 21101 & 1033 & 1.3 & Winter \\
\hline $2 / 10 / 2005$ & 6 & 1520 & 32 & 4437 & 795 & 3.6 & Winter \\
\hline $3 / 8 / 2005$ & 3 & 1220 & 20 & 2338 & 406 & 5.7 & Winter \\
\hline $3 / 28 / 2005$ & 12 & 1685 & 60 & 7675 & 3082 & 3.4 & Winter \\
\hline $4 / 20 / 2005$ & 12 & 480 & 15 & 4274 & 1017 & 5.9 & Spring \\
\hline $6 / 22 / 2005$ & 15 & 95 & 8 & 9120 & 266 & 4.0 & Summer \\
\hline $8 / 13 / 2005$ & 24 & 765 & 13 & 18408 & 514 & 10.0 & Summer \\
\hline $9 / 15 / 2005$ & 18 & 30 & 5 & 5518 & 86 & 10.0 & Fall \\
\hline $11 / 6 / 2005$ & 12 & 100 & 7 & 2801 & 135 & 10.8 & Fall \\
\hline $11 / 30 / 2005$ & 9 & 810 & 18 & 2503 & 363 & 5.0 & Fall \\
\hline $12 / 16 / 2005$ & 18 & 630 & 35 & 2561 & 458 & 5.5 & Winter \\
\hline $1 / 11 / 2006$ & 15 & 320 & 15 & 2903 & 214 & 5.8 & Winter \\
\hline $2 / 17 / 2006$ & 12 & 110 & 3 & 1493 & 37 & 2.5 & Winter \\
\hline $3 / 13 / 2006$ & 12 & 170 & 7 & 1345 & 75 & 2.5 & Winter \\
\hline $5 / 2 / 2006$ & 12 & 1920 & 60 & 4642 & 1331 & 7.0 & Spring \\
\hline $5 / 9 / 2006$ & 3 & 565 & 14 & 1621 & 322 & 5.6 & Spring \\
\hline $6 / 1 / 2006$ & 125 & 485 & 51 & 27194 & 930 & 10.7 & Summer \\
\hline $6 / 21 / 2006^{*}$ & 27 & 80 & 5 & 5190 & 93 & 4.7 & Summer \\
\hline $7 / 22 / 2006^{*}$ & 40 & 50 & 5 & 8333 & 81 & 7.5 & Summer \\
\hline $9 / 6 / 2006^{*}$ & 30 & 585 & 16 & 6087 & 410 & 4.5 & Fall \\
\hline
\end{tabular}

"Storms used for validation 


\section{Model Structure}

Typically, the pollutant accumulation and wash-off model can be described by the following equations (Shaheen, 1975; Chen and Adams, 2006):

$$
\begin{gathered}
\frac{d M_{a}}{d t}=k_{d}-k_{b} M_{a} \\
M_{w}=M_{a}\left(1-e^{-k_{w} R}\right)
\end{gathered}
$$

where $M_{a}$ is the amount of pollutant on the surface; $M_{w}$ is the amount of pollutant removed from the surface during a storm; $k_{d}$ is a constant rate of pollutant deposition; $k_{b}$ represents the pollutant removal rate due to wind and traffic; $k_{w}$ is a wash-off coefficient; $t$ is the antecedent dry period; and $R$ is the total runoff volume. Equation (2) is called a "first order" model because the exponent of the total runoff volume, $\mathrm{R}$, is 1 .

\section{Pollutant Accumulation Model}

A pollutant buildup model is required to estimate the mass of contaminants on impervious surfaces between storm events. The accumulation of contaminants follows an exponential increase as it approaches a maximum value $\left(M_{m}\right)$, regardless of the length of the dry period (Alley and Smith, 1981; Haiping and Yamada, 1996). Integration of Equation (1) results in the following equation:

$$
M_{a}=M_{m}\left(1-e^{-k_{b} t}\right)+M_{0} e^{-k_{b} t}
$$


where $M_{a}$ is the mass of pollutant on the parking lot surface $\left(g / \mathrm{m}^{2}\right) ; k_{b}$ is the pollutant removal rate $\left(\right.$ day $\left.^{-1}\right) ; M_{0}$ is the residual amount of pollutant after the previous runoff event $\left(\mathrm{g} / \mathrm{m}^{2}\right) ; M_{m}$ represents the maximum amount of pollutant buildup $\left(\mathrm{g} / \mathrm{m}^{2}\right)$; and $t$ is the antecedent dry period (days). These units will be used throughout the paper. For this study, $M_{0}$ was assumed to be zero. It was assumed that each runoff event had enough energy to remove the mass of contaminants accumulated on top of the impervious surface.

\section{Pollutant Wash-off Model}

The pollutant wash-off model describes the removal of contaminants from the impervious surface during a runoff event. Most typical wash-off models are a function of the total runoff volume or discharge (Sartor et al., 1976; Alley and Smith, 1981; Haiping and Yamada, 1998; Millar, 1999; Kanso et al., 2003; Rossman, 2004). In this study, the washed-off mass was assumed to be proportional to the available mass and to the discharge. The pollutant wash-off model can be written as follows:

$$
\frac{d M_{w}}{d t}=-k_{w} Q(t)^{w} M_{a}
$$

where $M_{w}$ represents the washed off mass $\left(g / \mathrm{m}^{2}\right)$ at time $t ; Q(t)$ the discharge $\left(\mathrm{m}^{3} /\right.$ day); $k_{w}$ is a wash-off coefficient (day $\left.{ }^{w-1} / m^{3 w}\right) ; M_{a}$ is the mass of pollutant on the parking lot surface $\left(g / \mathrm{m}^{2}\right)$; and $w$ is a wash-off exponent $(-)$. The wash-off exponent $w$ allows the model to have a non-linear dependency on the discharge, which could be convenient when the pollutograph is nonlinear. The pollutant concentration is calculated by dividing the eroded mass within a time interval $\Delta t$ by the runoff volume of that same interval. The 
accumulation (Equation (3)) and wash-off (Equation (4)) models can be used to obtain a single mathematical expression for continuous simulation (Chen and Adams, 2006; Haiping and Yamada, 1996). These two equations have to be multiplied by the watershed area if the total accumulated mass and total washed off mass are calculated.

\section{The Objective Function}

The aim of this study was to estimate the parameters that best fit the accumulation and wash-off model for four separate contaminants. The best fitting parameters were found by minimizing the sum of squares of residuals:

$$
O(\Theta)=\min \sum_{i=1}^{m}\left(C_{o b s}^{i}-C_{e s t}^{i}(\Theta)\right)^{2}
$$

where $O$ denotes the objective function, $\Theta$ represents the best fitting parameter values, $\mathrm{C}_{\mathrm{obs}}$ are the measured concentrations, $\mathrm{C}_{\text {est }}$ are the estimated concentrations when using $\Theta$, and $m$ is the number of samples analyzed during the storm event.

\section{Optimization Technique}

The accumulation and wash-off model was calibrated using field data and an optimization technique implemented to determine the most adequate fitting parameters for the model. Alley and Smith (1981) used Rosenbrock's method to find the best fit values for stormwater applications. A discussion on how parameter interaction affects the optimization technique was also provided. Gaume et al. (1998) investigated the uncertainty of the calibrated parameter values on an urban runoff model similar to 
equations (3) and (4). In their paper, the Powell method was used as the optimization technique and a description of the shape of the objective function was provided for several cases. One major difficulty was dealing with narrow valleys in the objective function, which would add more complexity to the search process. The lack of knowledge of the real pollutograph was a contributing factor to the failure of some optimization trials.

Simulated annealing (SA) was proposed by Kirkpatrick et al. (1983) and is a stochastic global optimization technique that finds the global minimum or maximum of a given mathematical function. The SA technique is meant to be used on highly non-linear multivariable problems. It was initially tested on physical applications but currently is extensively used in other scientific fields including hydrology. This technique has not yet been used on stormwater runoff models. The searching algorithm can be visualized as a bouncing sphere that can travel over the peaks and valleys of a given surface (the objective function). Throughout the iterative process the "energy" at which the sphere bounces decreases as it gets closer to the optimal value. In simulated annealing, a new trial solution (new set of parameter values) is accepted when there is a reduction in the current objective function value (Kirkpatrick, 1983). First, the probability of accepting a new trial solution is calculated as follows:

$$
p(\Delta O)=e^{-\frac{\Delta O}{T}}
$$

where $\Delta O$ is the change in the objective function, $T$ is a parameter called temperature (which represents the energy of the bouncing sphere), and $p(\Delta O)$ is the probability of accepting the proposed set of parameter values. At higher "temperatures" the algorithm extensively explores the parameter space so the global minimum is likely to be found. 
When the temperature is high the probability of accepting the proposed set of parameters is high as well. As the temperature drops, the probability of accepting new candidates reduces and the search focuses on previous local optimal parameter values.

The value of $T$ is initially set high and is periodically reduced according to a "cooling schedule" (Hopgood, 2001) with a maximum number of steps $s_{t}$. A reduction coefficient 0 $<a<1$ is used to slowly decrease the value of $\mathrm{T}$. A commonly used, simple cooling schedule is:

$$
T_{i+1}=a T_{i} \quad i=0,1, \ldots, s_{t}
$$

The SA annealing algorithm overcomes the problem of being trapped in a local minimum by accepting a trial solution that, although is not the best, may lead to the true optimal values. Large $s_{t}$ values allow the control variable to decrease slowly and then perform the search on a broad area. In this study, the following annealing schedule was applied: $T=20000, a=0.9$, and $s_{t}=100$. The annealing schedule determines the degree of uphill (or downhill) movement permitted during the search so it is critical to the algorithm's performance. These parameters are problem-specific and depend on the scaling of the change in the objective function $\Delta \mathrm{O}$. For this study, various annealing schedules were tested several times until the same optimal values were achieved.

The total number of iterations was fixed $\left(5 \times 10^{4}\right)$ and the optimization process was stopped when the new set of parameter values did not change over 100 iterations. If these parameter values were still changing by the end of the total number of iterations, then the model was re-run again with a higher number of iterations $\left(1 \times 10^{5}\right)$.

Bounds were necessary for the practical implementation of the SA method. The upper and lower bounds for all the parameters are given in Table 2. These values were determined after performing some trial runs with the data, and recommendations from 
the literature (Haiping et al., 1996; Chen et al., 2006; Guame et al., 1998). SA results were compared to the Table 2 values to verify that the results were not close to the upper bounds.

Table 2 Bounds of calibration parameters. Units: $M_{m}\left(g / m^{2}\right), k_{b}\left(\right.$ day $\left.^{-1}\right), k_{w}\left(\right.$ day $\left.^{w-1} / m^{3 w}\right)$, and $w(-)$.

\begin{tabular}{|c|c|c|c|c|c|c|c|c|}
\hline \multirow{2}{*}{ Contaminant } & \multicolumn{2}{|c|}{$M_{m}$} & \multicolumn{2}{|c|}{$k_{b}$} & \multicolumn{2}{|c|}{$k_{w}$} & \multicolumn{2}{|c|}{$w$} \\
\hline & Lower & Upper & Lower & Upper & Lower & Upper & Lower & Upper \\
\hline TSS & 0 & 10 & 0 & 0.2 & 0 & 0.2 & 0 & 2 \\
\hline TPH-D & 0 & 0.2 & 0 & 0.01 & 0 & 0.01 & 0 & 2 \\
\hline DIN & 0 & 0.2 & 0 & 0.01 & 0 & 0.01 & 0 & 2 \\
\hline $\mathrm{Zn}$ & 0 & 0.2 & 0 & 0.01 & 0 & 0.01 & 0 & 2 \\
\hline
\end{tabular}

\section{Concentration Values Below Detection Limit}

A concentration value below the analytical detection limit (BDL) is reported when pollutant concentrations are below the analytical reporting limit of the laboratory. The application of the mathematical model requires finite concentration values. The researcher is left with few options when this situation arises: discard valuable information provided by the BDL samples or select an estimate of the concentration $(1 / 2$ and 0 the detection limit is common). A method for generating estimates is to fit a probability distribution based on the data above the reporting limit (Helsel and Hirsch, 2002). First, a distribution shape is assumed and then the distribution parameters are computed by using conventional methods such as Maximum Likelihood Estimator (MLE) or probability plot procedures. Finally, discrete BDL concentrations (values between 0 and the detection limit) can be randomly generated using the fitted distribution. Table 3 summarizes the number of samples reported as BDL for each contaminant. In this study, 
the detection limits were typically $0.50 \mathrm{mg} / \mathrm{l}$ for TPH-D, $0.03 \mathrm{mg} / \mathrm{l}$ for $\mathrm{Zn}, 0.05 \mathrm{mg} / \mathrm{l}$ for DIN, and $10 \mathrm{mg} / \mathrm{l}$ for TSS. However, the reported detection limit could vary depending on the actual sample volume and concentrations.

Table 3 Summary of samples with concentrations reported as BDL.

\begin{tabular}{cccccc}
\hline Format & $\begin{array}{c}\text { Total Number } \\
\text { of Samples }\end{array}$ & TPH-D & Zn & DIN & TSS \\
\hline Discrete & 234 & 69 & 34 & 68 & 38 \\
Percentage & $100 \%$ & $29 \%$ & $15 \%$ & $29 \%$ & $16 \%$ \\
\hline
\end{tabular}

A gamma distribution was employed for this study since it adequately described the collected data (other distributions such as gumbel and log-normal were tested). The data consisted of the individual concentration values obtained for the storms summarized in Table 1 , which were assumed to be independent. The parameters, $\gamma$ (shape) and $\theta$ (scale), of the distribution were determined by using the MLE method and are shown in Table 4. The probability density functions (PDFs) for each contaminant are shown in Figure 1 as well as the respective cumulative probability function (CDFs). The Kolmogorov-Smirnov test (KS-test) was used to measure the goodness-of-fit of the fitted probability distribution. A good fit was obtained for TPH-D, Zn, and DIN (Figure 1.b, 1.d, and 1.f) since $D_{n, \alpha} \leq D_{\text {critical }}$. The $D_{n, \alpha}$ statistic was computed for a level of significance $\alpha=$ 0.05 (95\% confidence interval) and $n$ concentration values above the detection limit. The D statistic represents the maximum vertical deviation between the empirical and theoretical data probability distributions. Although for TSS the fitted distribution did not pass the KS-test at 0.05 level of significance, the fitted probability distribution was utilized for practical purposes. 
Table 4 Parameters of the gamma distribution fitted to the data.

\begin{tabular}{cccccc}
\hline Contaminant & $\begin{array}{c}\text { Total samples } \\
\text { above DL }(\mathbf{n})\end{array}$ & $\gamma$ (shape) & $\theta$ (scale) & $\mathrm{D}_{\text {critical }}$ & $\mathrm{D}_{\mathrm{n}, \alpha}$ \\
\hline TPH-D & 165 & 2.82 & 0.39 & 0.11 & 0.11 \\
Zn & 200 & 1.83 & 0.04 & 0.10 & 0.10 \\
DIN & 166 & 2.08 & 0.31 & 0.11 & 0.10 \\
TSS & 196 & 0.79 & 82.80 & 0.10 & 0.14 \\
\hline
\end{tabular}

Level of significance $\alpha=0.05$

This statistical method is meant to preserve the entire distribution of the data both below and above the detection limit. The more data points above the reporting limit, the better the quality of the model from a statistical point of view. For TPH-D and DIN the shape parameter $(\gamma)$ is likely to be much smaller due to the fact that nearly $30 \%$ of the concentrations were reported below detection limit. 

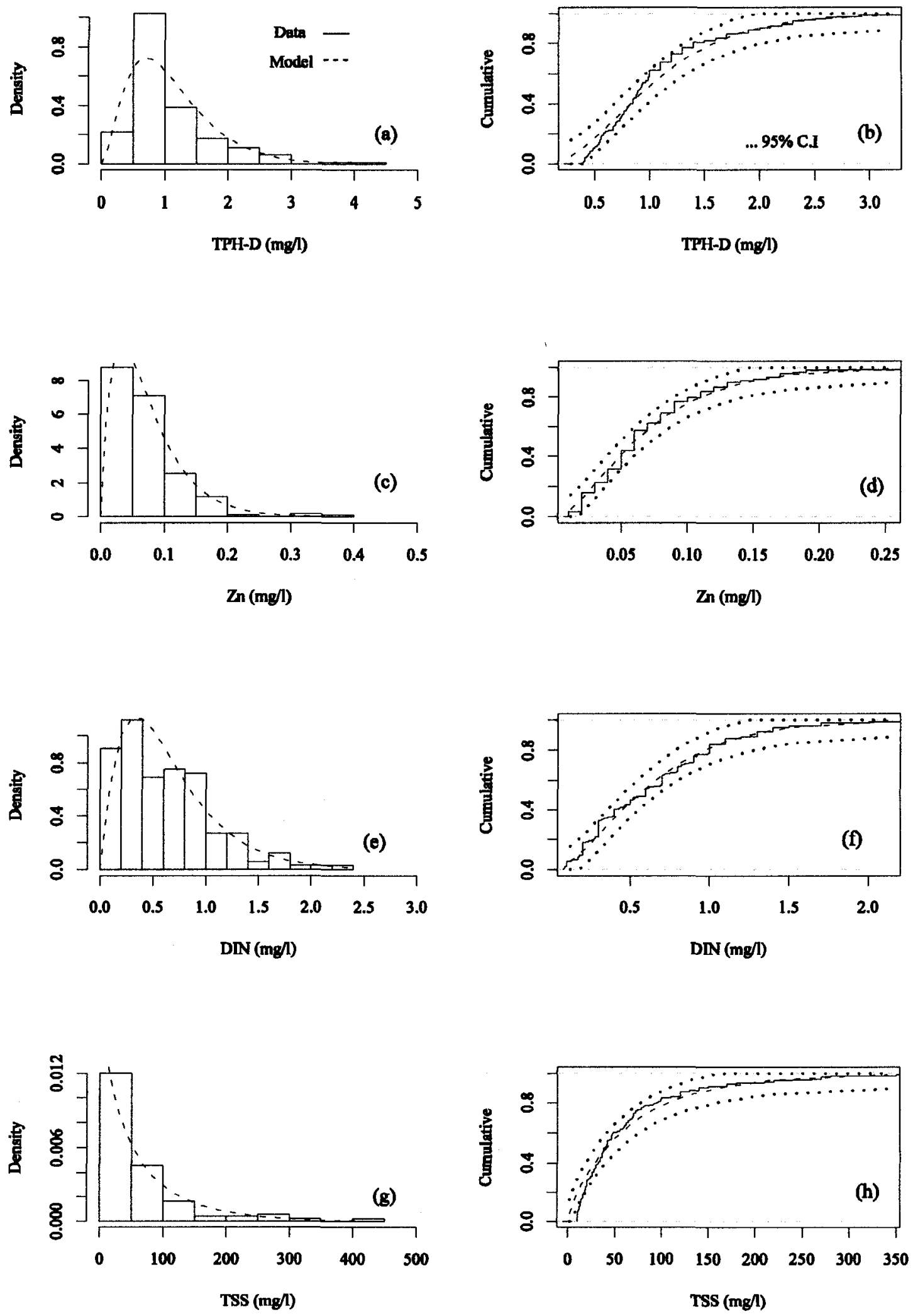

Figure 1 Empirical and theoretical data probability distributions. C.I: Confidence Interval. 


\section{Calibration Procedure}

A broad range of storms (Table 1) were independently used to obtain the optimal values of model parameters for each contaminant. The calibration was performed under the assumption of null residual mass after each rain event $\left(M_{0}=0\right)$. This assumption is not true when the storm event is flow-limited, in other words, when the storm is not intense enough and with enough volume to wash off the mass of contaminants on the surface. A continuous simulation approach was not performed because inter-event samples were not available. Additionally, not all storm events were monitored. The null residual mass assumption was verified by comparing the total available mass $\left(M_{a}\right)$ and the mass that was actually washed off $\left(M_{w}\right)$. Initial parameter values were randomly generated using the bounds shown in Table 2. The root mean square error (RMSE) was used as the quality criterion. When changes in the objective function remained significant, the model was re-run and the total number of iterations increased. On average, convergence was achieved after $2 \times 10^{4}$ iterations. For calibration, concentration values were used as they were reported from the lab and no further statistical analysis was applied to discard anomalous data. The calibration period included storms from 09/08/2004 to 6/01/2006. The last three storm events described in Table 1 were utilized for validation.

\section{Results}

\section{Model Application}

Figures 2 through 4 illustrate model results for some of the storms considered in this study. Each figure shows the hydrograph, hyetograph, observed concentrations, estimated concentrations, and the respective relative cumulative mass (CDF). The CDF 
for each contaminant is a mass-based CDF and was obtained by multiplying the estimated concentrations by the synoptically measured flows. For example, Figure 2(a) shows flow rate changes (below) and rainfall pattern (top). The CDFs for each contaminant are shown in Figure 2(b). Figures 2(c), 2(d), 2(e), and 2(f) show observed and estimated concentrations for DIN, TPH-D, TSS, and Zn (RMSEs are reported).

The 03/08/2005 event (Figure 2) had an almost constant rainfall intensity, and as a result, the hydrograph increased and decreased gradually. The model with calibrated parameters does a very good job for reproducing most of the observed data. The observed concentrations and pollutograph for TPH-D stand out from the other contaminants due to the poorer fit and the scatter of the data. The last seven samples for DIN were reported BDL $(<0.5 \mathrm{mg} / \mathrm{l})$. This explains the scatter of the observed values on the lower part of the graph since those values were randomly generated. Nevertheless, the smoothness of the estimated pollutograph does not seem to be affected. For this storm, BDL values were not recorded for TSS, $\mathrm{Zn}$, and TPH-D. The CDFs look very similar for all contaminants. The parameter $w$ was close to 1 because observed concentrations decreased smoothly. Optimized parameter values for the 03/08/2005 storm event are presented in Table 5. 

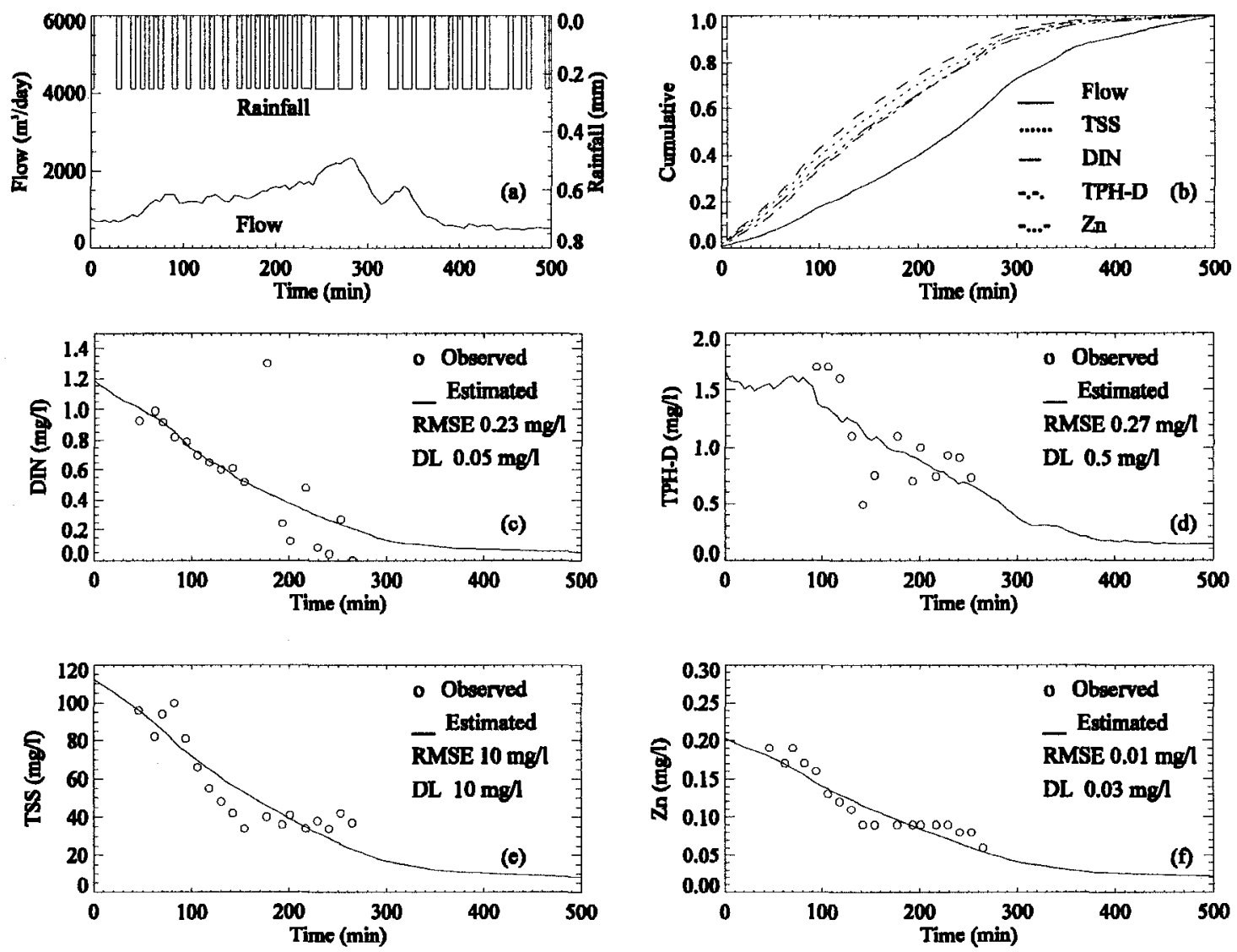

Figure 2 Results for the 03/08/2005 storm. Optimized parameter values are presented in Table 5. Cumulative concentrations (b) were computed using estimated values. 
Table 5 Optimized parameter values for selected storm events. Units: $M_{m}\left(g / m^{2}\right), k_{b}$ (day $\left.{ }^{1}\right), k_{w}\left(d a y^{w-1} / m^{3 w}\right)$, and $w(-)$.

\begin{tabular}{|c|c|c|c|c|c|}
\hline \multirow{2}{*}{$\begin{array}{l}\text { Rainfall Event } \\
\mathrm{m} / \mathrm{d} / \mathrm{y}\end{array}$} & \multirow{2}{*}{ Parameter } & \multicolumn{4}{|c|}{ Contaminant } \\
\hline & & DIN & TPH-D & TSS & $\mathrm{Zn}$ \\
\hline \multirow[t]{4}{*}{$3 / 8 / 2005$} & $M_{m}$ & 0.1513 & 0.1858 & 0.7119 & 0.0845 \\
\hline & $k_{b}$ & 0.0052 & 0.0093 & 0.1896 & 0.0021 \\
\hline & $k_{w}$ & 0.0046 & 0.0005 & 0.0079 & 0.0054 \\
\hline & $w$ & 1.0684 & 1.3504 & 0.9711 & 1.0013 \\
\hline \multirow[t]{4}{*}{$4 / 20 / 2005$} & $M_{m}$ & 0.1944 & 0.1993 & 1.7269 & 0.1238 \\
\hline & $k_{b}$ & 0.0099 & 0.0094 & 0.0980 & 0.0024 \\
\hline & $k_{w}$ & 0.0026 & 0.0009 & 0.0038 & 0.0050 \\
\hline & $w$ & 1.1322 & 1.1925 & 1.1539 & 1.0341 \\
\hline \multirow[t]{4}{*}{$1 / 11 / 2006$} & $M_{m}$ & 0.0207 & 0.1866 & 0.3383 & 0.0681 \\
\hline & $k_{b}$ & 0.0095 & 0.0096 & 0.1918 & 0.0004 \\
\hline & $k_{w}$ & 0.0092 & 0.0000 & 0.0057 & 0.0006 \\
\hline & $w$ & 1.2357 & 1.8571 & 1.1560 & 1.5140 \\
\hline
\end{tabular}

A type of first flush event is demonstrated in Figure 3 for the 04/20/2005 storm. The highest intensity occurred early in the storm and a constant rainfall intensity occurred during the rest of the storm duration. The first flush effect is more pronounced for the total suspended solids CDF. BDL concentration values were reported for DIN, TSS, and Zn for the last 4, 3, and 2 samples, respectively. Only one BDL value was reported for TPH-D at 356 min. The estimated pollutograhs match the higher concentrations at the beginning of the storm. For TPH-D, observed concentrations increase at the very end of the event, however, the model is not capable of estimating this phenomenon. The parameter $w$ ranged from 1.0 and 1.2 for the different contaminants due to the smooth decrease in observed concentrations. 

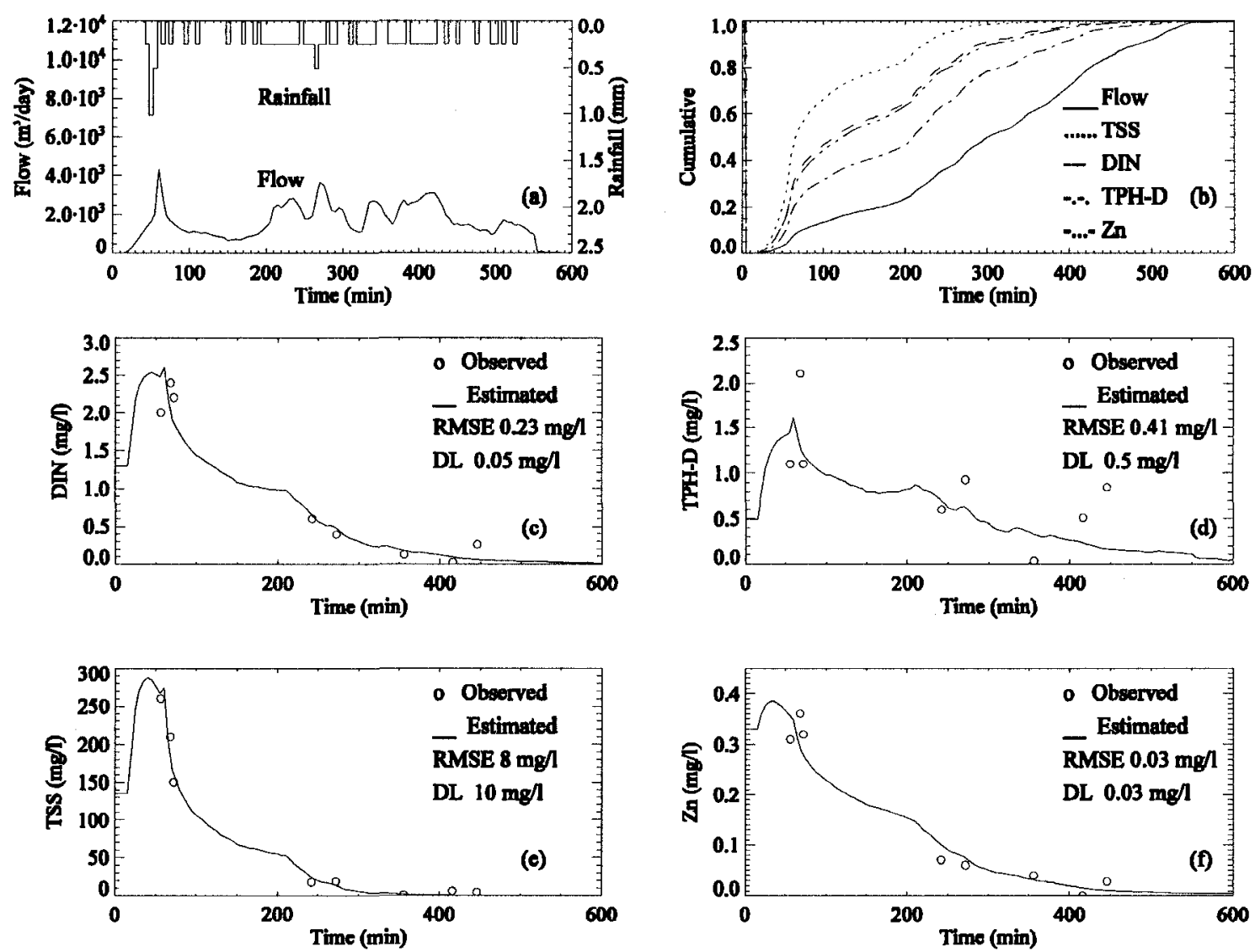

Figure 3 Results for the 04/20/2005 storm. Optimized parameter values are presented in

Table 5. Cumulative concentrations (b) were computed using estimated values.

The 01/11/2006 storm was a late-peaking event (Figure 4). At least two peaks were observed in the measured concentrations for all the contaminants, one approximately at $350 \mathrm{~min}$ and other at $550 \mathrm{~min}$, in direct response to rainfall intensity peaks. BDL values were reported for $\mathrm{Zn}$ and DIN for samples taken at 501 and $601 \mathrm{~min}$. Even though the peak flow occurred towards the end of the event, the highest concentration values were measured at the beginning of the storm. The parameter $w$ ranged from 1.2 to 1.9 for the different contaminants, which agrees with the non-linear trend observed in the data. 

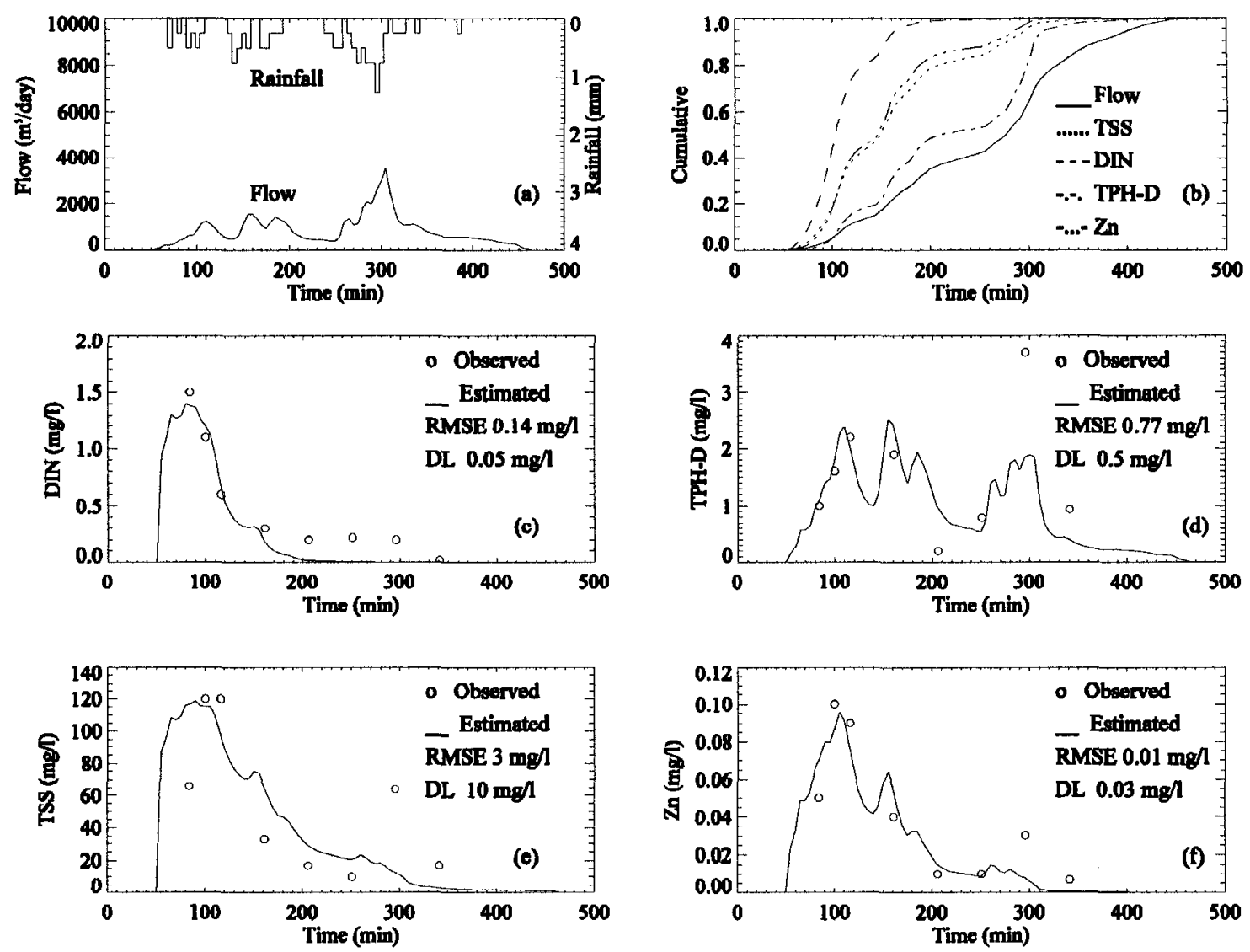

Figure 4 Results for the 01/11/2006 storm. Optimized parameter values are presented in Table 5. Cumulative concentrations (b) were computed using estimated values.

In general, the highest concentrations were observed at the beginning of the storm and few samples had values reported as BDL towards the end of the event. The storm events that are not shown possessed similar patterns.

Figure 5 shows the changes in the objective function (Equation (5)) when the parameters were calibrated for $\mathrm{Zn}$ on the $03 / 13 / 2006$ event. During the first iterations, high values for the objective function were obtained due to the fact that the SA algorithm was randomly exploring the parameters space. After $1 \times 10^{4}$ iterations the objective 
function seemed to have reached its minimum value. The optimization process was stopped at $1.2 \times 10^{4}$ iterations.
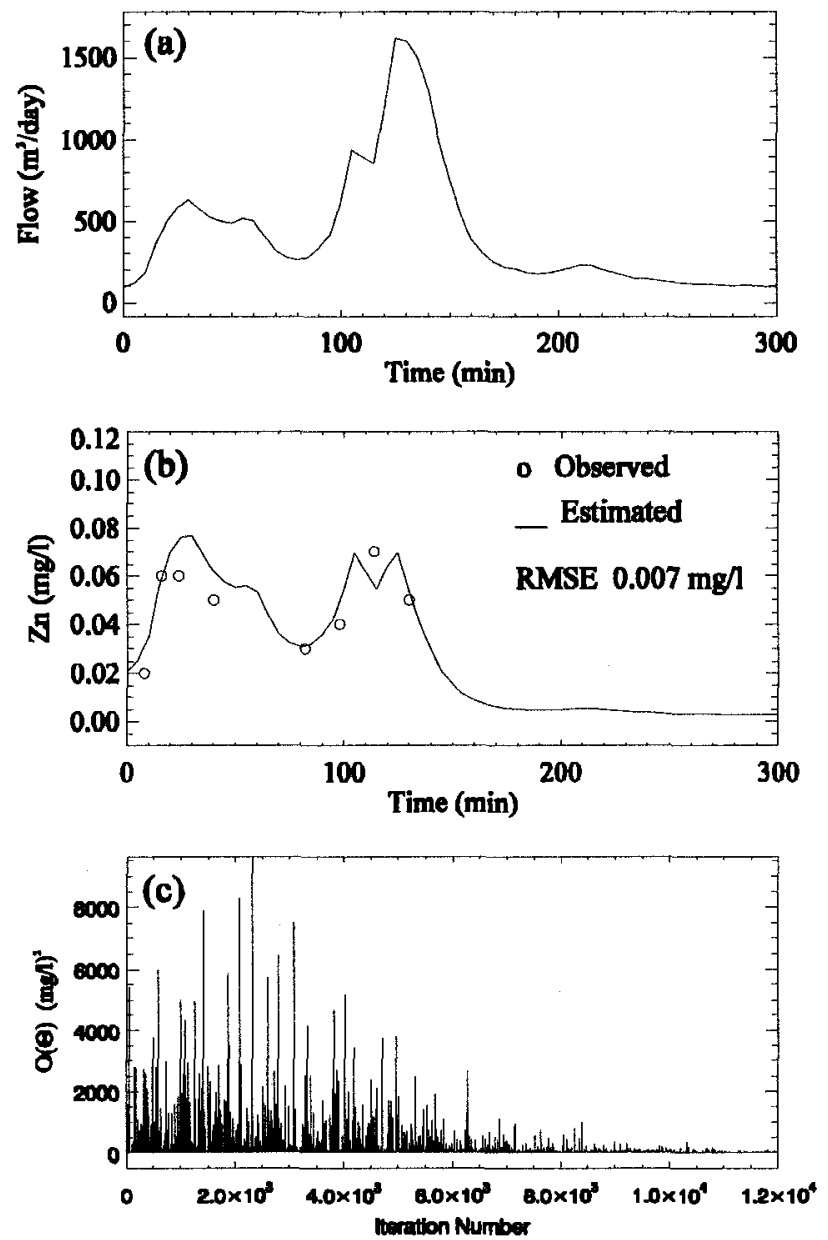

Figure 5 Optimization process and calibration results for the 03/13/2006 storm. Optimized parameter values are shown for zinc. Optimized parameters: $M_{m}=0.13 \mathrm{~g} / \mathrm{m}^{2}$, $k_{b}=0.0004$ day $^{-1}, k_{w}=0.0002$ day $^{w-1} / m^{3 w}, w=1.71$, and $b=2.50$ days (antecedent dry period).

Box and whisker plots of the optimized parameter values for all the storms and contaminants of the study are presented in Figure 6. Gaume et al. (1998) found fitting 
parameter values after calibrating a similar runoff water quality model for suspended solids. In their study, the parameter values ranged as follows: $40-120 \mathrm{~g} / \mathrm{m}^{2}$ for $\mathrm{M}_{\mathrm{m}}, 0.2$ -0.8 day $^{-1}$ for $k_{b}, 0.005-0.030$ day $^{w-1} / m^{3 w}$ for $k_{w}$, and $1.0-2.0$ for $w$. Note that their model was calibrated for an urban sewer system so this could explain why some parameter values are different from those provided in this paper. For example, Figure 7 shows a range of $0.3-1.9 \mathrm{~g} / \mathrm{m}^{2}$ for $M_{m}$, which is smaller than the one provided by Gaume et al. (1998) for suspended solids.
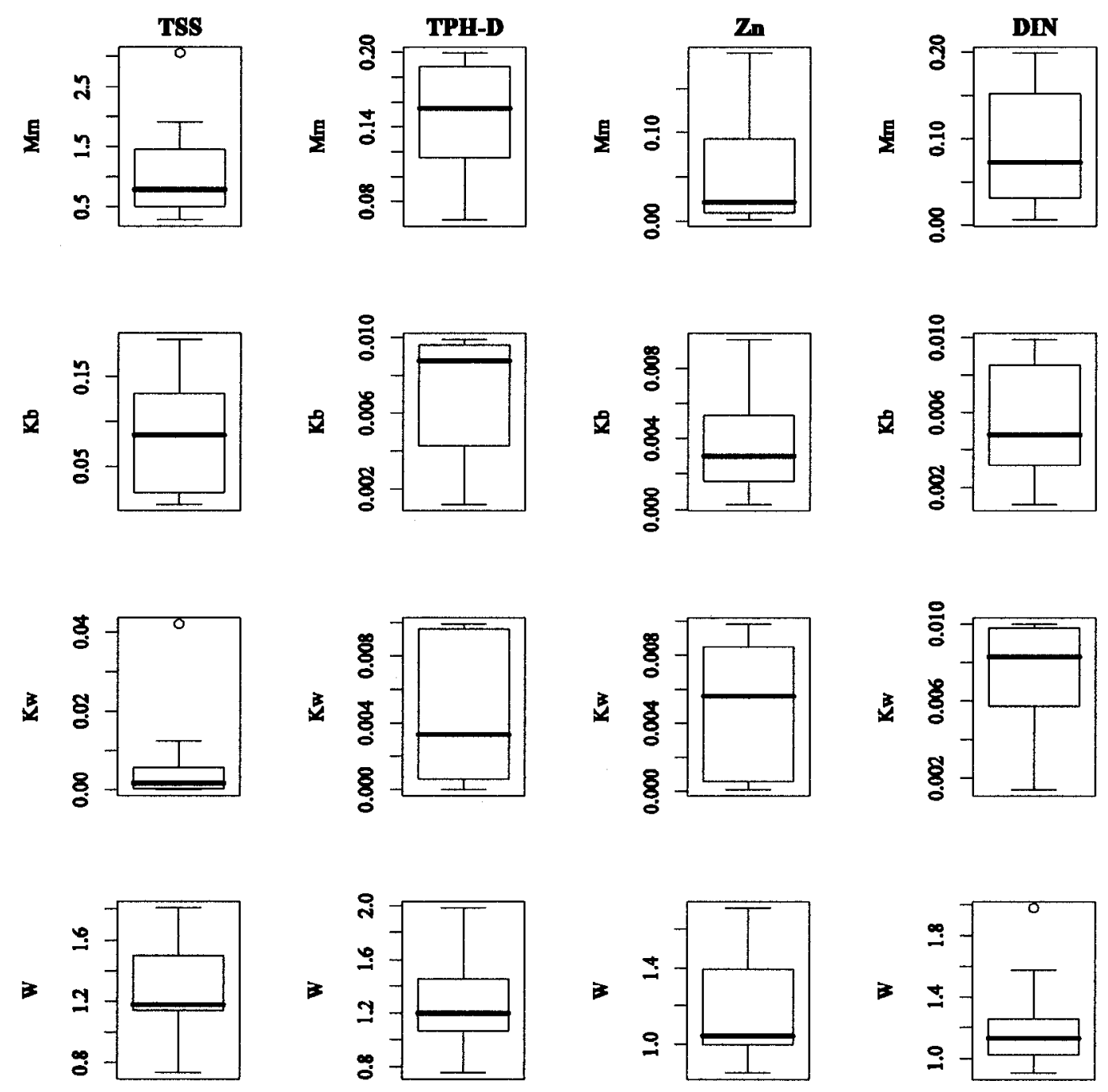

Figure 6 Box and whisker plot of the optimized parameter values. Units: $M_{m}\left(g / m^{2}\right), k_{b}$ $\left(\right.$ day $\left.^{-1}\right), k_{w}\left(\right.$ day $\left.^{w-1} / m^{3 w}\right)$, and $w(-)$. 


\section{Statistical Analysis}

Figure 7 shows the CDFs obtained for the different model parameters (optimized values) and pollutants. A normal distribution was fitted to these CDFs and a $95 \%$ confidence interval ( $\alpha=0.05$ level of significance) was computed to measure the goodness-of-fit of the distribution. For this purpose, the KS-test was used. The mean $\bar{x}$ and the standard deviation (s) of the fitted normal distributions are shown in Table 6 . The distribution of the parameters $M_{m}$ (for $Z n$ ), $k_{w}$ (for DIN), $k_{w}$ (for TSS) did not pass the KStest. Additionally, only 22 optimized parameter values were used for TPH-D and TSS since calibration failed for the 1/14/2005 (TPH-D) and 5/2/2006 (TSS) storms. 

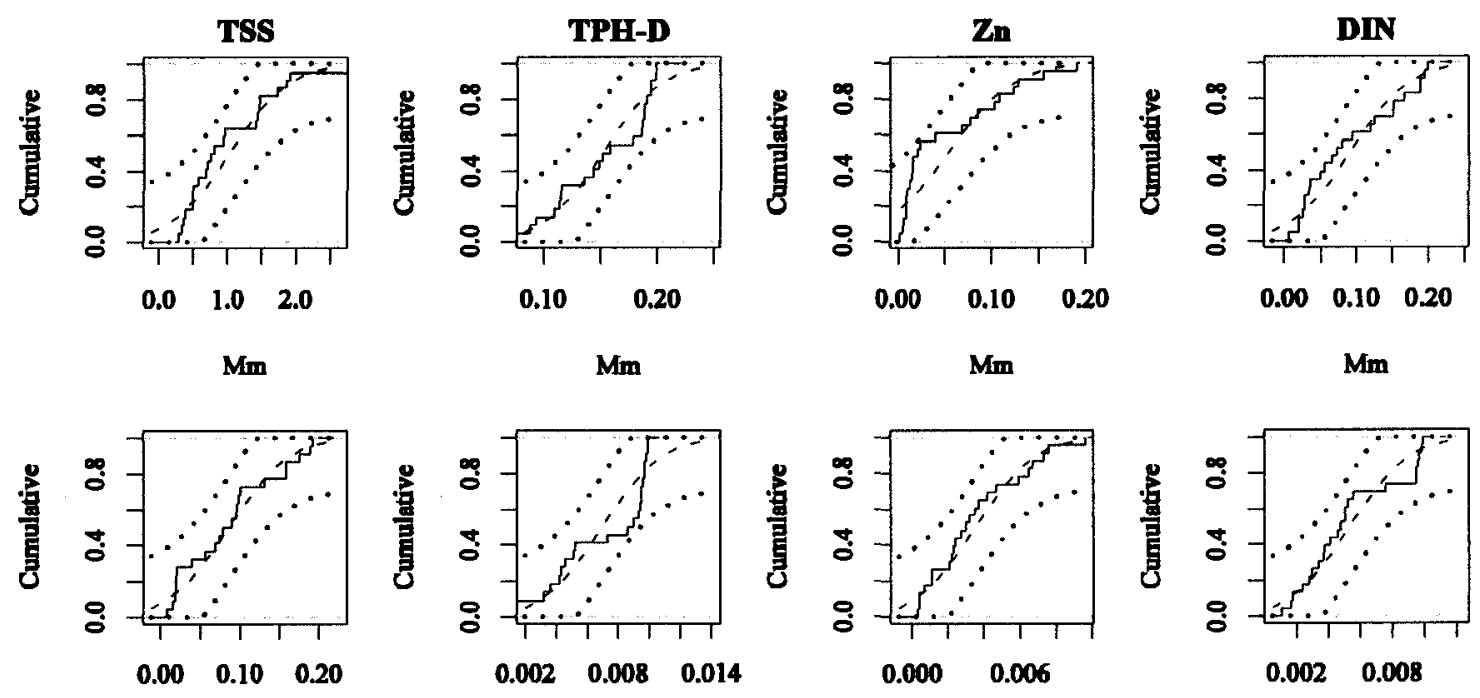

$\mathbf{K b}$
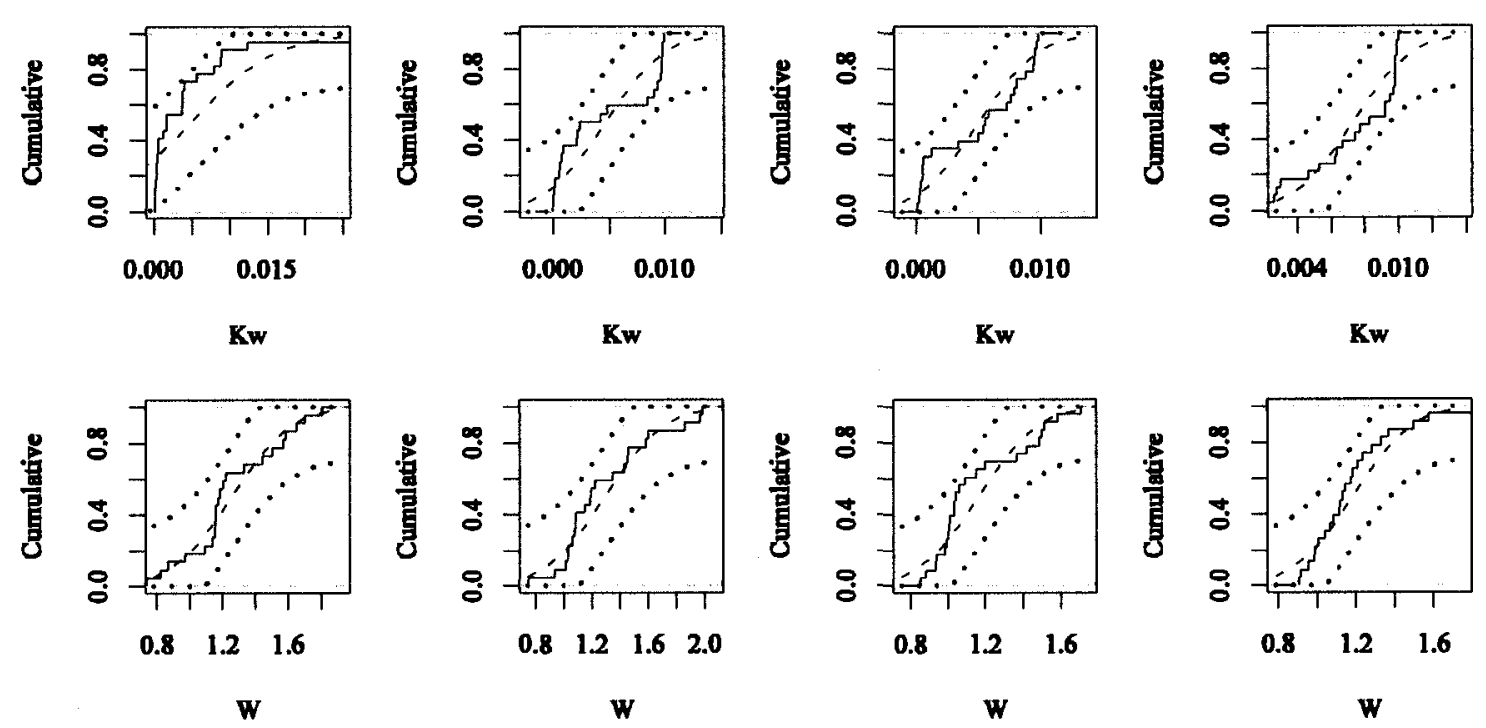

Figure 7 CDFs of optimized model parameter values (solid line) and fitted normal CDFs (dashed line). A $95 \%$ confidence interval is reported (dotted line). Units: $M_{m}\left(g / m^{2}\right), k_{b}$ $\left(\right.$ day $\left.^{-1}\right), k_{w}\left(\right.$ day $\left.^{w-1} / m^{3 w}\right)$, and $w(-)$.

The mean $M_{m}$ value $\left(1.029 \mathrm{~g} / \mathrm{m}^{2}\right)$ was considerable higher for TSS than for the other contaminants. This agrees with the fact that, for this site, observed TSS concentrations were on average higher than those for Zn, TPH-D, and DIN. This characteristic was also reported by Chen and Adams (2006) when a similar stormwater model was applied on 
total phosphorus, copper, total kjeldahl nitrogen (TKN), and TSS. Chen and Adams also reported a range of variation for $M_{m}$ between 0.018 and $25 \mathrm{~g} / \mathrm{m}^{2}$. The watershed used in their study was composed of residential and commercial land use.

Table 6 Parameters of the normal distribution fitted to the optimized model parameter values. Units: $M_{m}\left(g / m^{2}\right), k_{b}\left(\right.$ day $\left.^{-1}\right), k_{w}\left(\right.$ day $\left.^{w-1} / m^{3 w}\right)$, and $w(-)$.

\begin{tabular}{|c|c|c|c|c|c|}
\hline Parameter & Statistic & TSS & TPH-D & $\mathrm{Zn}$ & DIN \\
\hline \multirow[t]{5}{*}{$M_{m}$} & $\bar{x}$ & 1.029 & 0.152 & 0.053 & 0.091 \\
\hline & s & 0.693 & 0.042 & 0.056 & 0.066 \\
\hline & $n$ & 22 & 22 & 23 & 23 \\
\hline & $D_{\text {critical }}$ & 0.281 & 0.281 & 0.275 & 0.275 \\
\hline & $D_{n, a}$ & 0.178 & 0.202 & 0.270 & 0.153 \\
\hline \multirow[t]{5}{*}{$k_{b}$} & $\bar{x}$ & 0.086 & 0.007 & 0.004 & 0.005 \\
\hline & $\mathbf{s}$ & 0.060 & 0.003 & 0.003 & 0.003 \\
\hline & $n$ & 22 & 22 & 23 & 23 \\
\hline & $D_{\text {critical }}$ & 0.281 & 0.281 & 0.275 & 0.275 \\
\hline & $D_{n, \alpha}$ & 0.139 & 0.246 & 0.129 & 0.179 \\
\hline \multirow[t]{5}{*}{$k_{w}$} & $\bar{x}$ & 0.005 & 0.005 & 0.005 & 0.007 \\
\hline & s & 0.009 & 0.004 & 0.004 & 0.003 \\
\hline & $n$ & 22 & 22 & 23 & 23 \\
\hline & $D_{\text {critical }}$ & 0.281 & 0.281 & 0.275 & 0.275 \\
\hline & $D_{n, a}$ & 0.297 & 0.217 & 0.194 & 0.428 \\
\hline \multirow[t]{5}{*}{$w$} & $\bar{x}$ & 1.255 & 1.298 & 1.166 & 1.187 \\
\hline & $\mathbf{s}$ & 0.290 & 0.335 & 0.252 & 0.244 \\
\hline & $n$ & 22 & 22 & 23 & 23 \\
\hline & $\mathrm{D}_{\text {critical }}$ & 0.281 & 0.281 & 0.275 & 0.275 \\
\hline & $D_{n, \alpha}$ & 0.184 & 0.175 & 0.226 & 0.163 \\
\hline
\end{tabular}

A correlation analysis was performed to study the degree of linear dependencies among the model parameters. Table 7 shows the correlation coefficients for the different parameter combinations and pollutants. A negative correlation value was obtained 
between $k_{w}$ and $w$ for all the contaminants; however, this can be explained by the mathematical formulation of the model ( $k_{w}$ is a coefficient and $w$ is an exponent). It was found some positive correlation between $M_{m}$ and $k_{b}$ for TSS, TPH-D, and DIN. Similar results were reported by Kanso et al. (2003), where a positive correlation was obtained between $M_{m}$ and $k_{b}$ for suspended solids.

Table 7 Correlation matrixes arranged by pollutant. Units: $M_{m}\left(g / m^{2}\right), k_{b}\left(\right.$ day $\left.^{-1}\right), k_{w}\left(d a y^{w-}\right.$ $\left.1 / m^{3 w}\right)$, and $w(-)$.

\begin{tabular}{cccccc} 
Pollutant & Parameter & Mm & $k_{b}$ & $k_{w}$ & $w$ \\
\hline TSS & $M m$ & 1 & & & \\
& $k_{b}$ & 0.38 & 1 & & \\
& $k_{w}$ & 0.03 & -0.18 & 1 & \\
& $w$ & -0.12 & -0.08 & -0.56 & 1 \\
\hline TPH-D & $M m$ & 1 & & & \\
& $k_{b}$ & 0.19 & 1 & & \\
& $k_{w}$ & -0.27 & -0.53 & 1 & \\
& $w$ & 0.32 & 0.02 & -0.55 & 1 \\
\hline Zn & $M m$ & 1 & & & \\
& $k_{b}$ & -0.39 & 1 & & \\
& $k_{w}$ & -0.51 & 0.16 & 1 & \\
& $w$ & 0.31 & -0.51 & -0.66 & 1 \\
\hline DIN & $M m$ & 1 & & & \\
& $k_{b}$ & 0.10 & 1 & & \\
& $k_{w}$ & -0.31 & -0.21 & 1 & \\
& $w$ & -0.59 & -0.12 & -0.38 & 1
\end{tabular}

\section{Monte Carlo Simulation}

A Monte Carlo simulation was performed to evaluate the ability of the model to predict pollutant concentrations at the catchment's outlet. Three storms were selected for 
validation: 06/21/2006, 07/22/2006, and 9/6/2006. However, only results for the first two storms are shown in this paper. The fitted normal distributions to the optimized parameter values (Table 6 and Figure 6 ) were used to generate $M_{m}, k_{b}, k_{w}$, and $w$. The parameters were assumed to be independent and non-correlated for modeling purposes. Note that a multivariate test of independence was not performed for this study. Further research should explore the use of statistical tools such as the Generalized Likelihood Uncertainty Estimator (GLUE) when analyzing the uncertainties of the wash-off model. The GLUE procedure (Beven and Binley, 1992) implicitly incorporates the correlation structure of the model since it evaluates a set of fitting parameters rather than individual values.

Simulation results are shown in Figure 8 and Figure 9. The central solid line indicates the median of the simulated values. The $10\left(q_{10}\right), 30\left(q_{30}\right), 70\left(q_{70}\right)$, and $90\left(q_{90}\right)$ percentiles were selected as a measure of uncertainty. The number of simulations was set to 5,000 for each contaminant. Figure 8 presents results for the 06/21/2006 storm. An acceptable performance of the model is achieved if observed concentration values fall within the uncertainty limits. The maximum observed concentration for TPH-D, TSS, and $\mathrm{Zn}$ was obtained for the first sample; however, the model was not able to adequately estimate these concentrations for TPH-D and TSS. DIN observed concentrations fell within the $30 \%$ and $70 \%$ uncertainty limits. Zinc observed concentrations fell within the $10 \%$ and $90 \%$ uncertainty limits. 

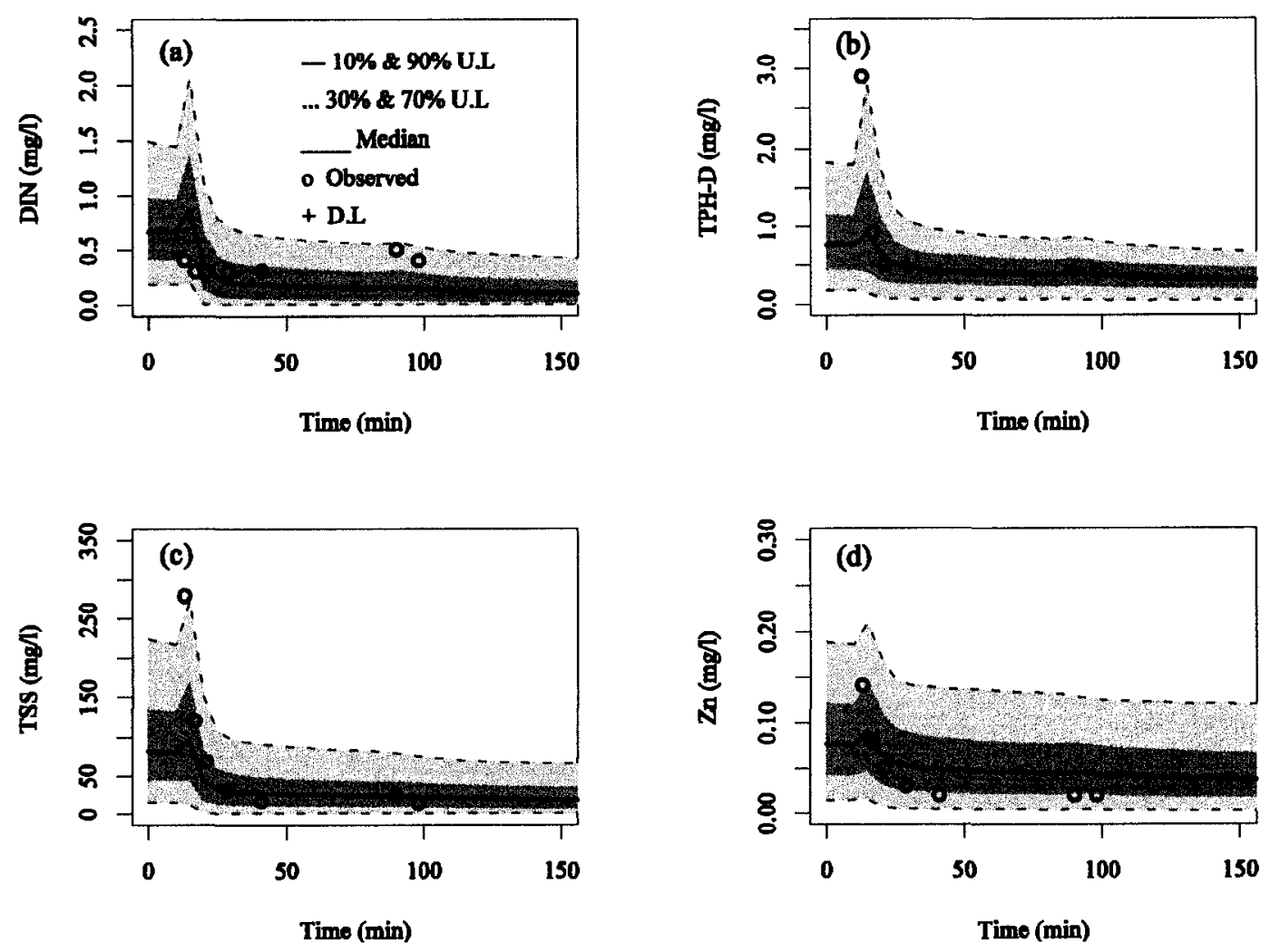

Figure 8 Monte Carlo simulations of predicted pollutant concentrations at the catchment's outlet for the 06/21/2006 storm event. U.L: Uncertainty Limits. The dark shaded region indicates the $30 \%$ and $70 \%$ uncertainty limits. The light grey shaded region indicates the $10 \%$ and $90 \%$ uncertainty limits. Observed concentrations values and the reported detection limits were plotted as discrete points.

Monte Carlo simulations for the 07/22/2006 event are displayed in Figure 9. For DIN, the first three observed concentrations fell outside the plotted uncertainty limits (Figure 9(a)). This indicates poor performance of the model. However, the remaining observed concentrations are much closer to the expected concentrations. Results for TPH-D fell within the $30 \%$ and $70 \%$ uncertainty limits and the expected concentration line predicts very well some observed concentrations (Figure 9(b)). Results for TSS tend to overestimate pollutant concentrations since there are six observed values that fell below 
the $30 \%$ uncertainty limit (Figure 9(c)). For $\mathrm{Zn}$, four samples fell within the $30 \%$ and 70 $\%$ uncertainty limits and the remaining values below the $30 \%$ uncertainty limit (Figure 9(d)).
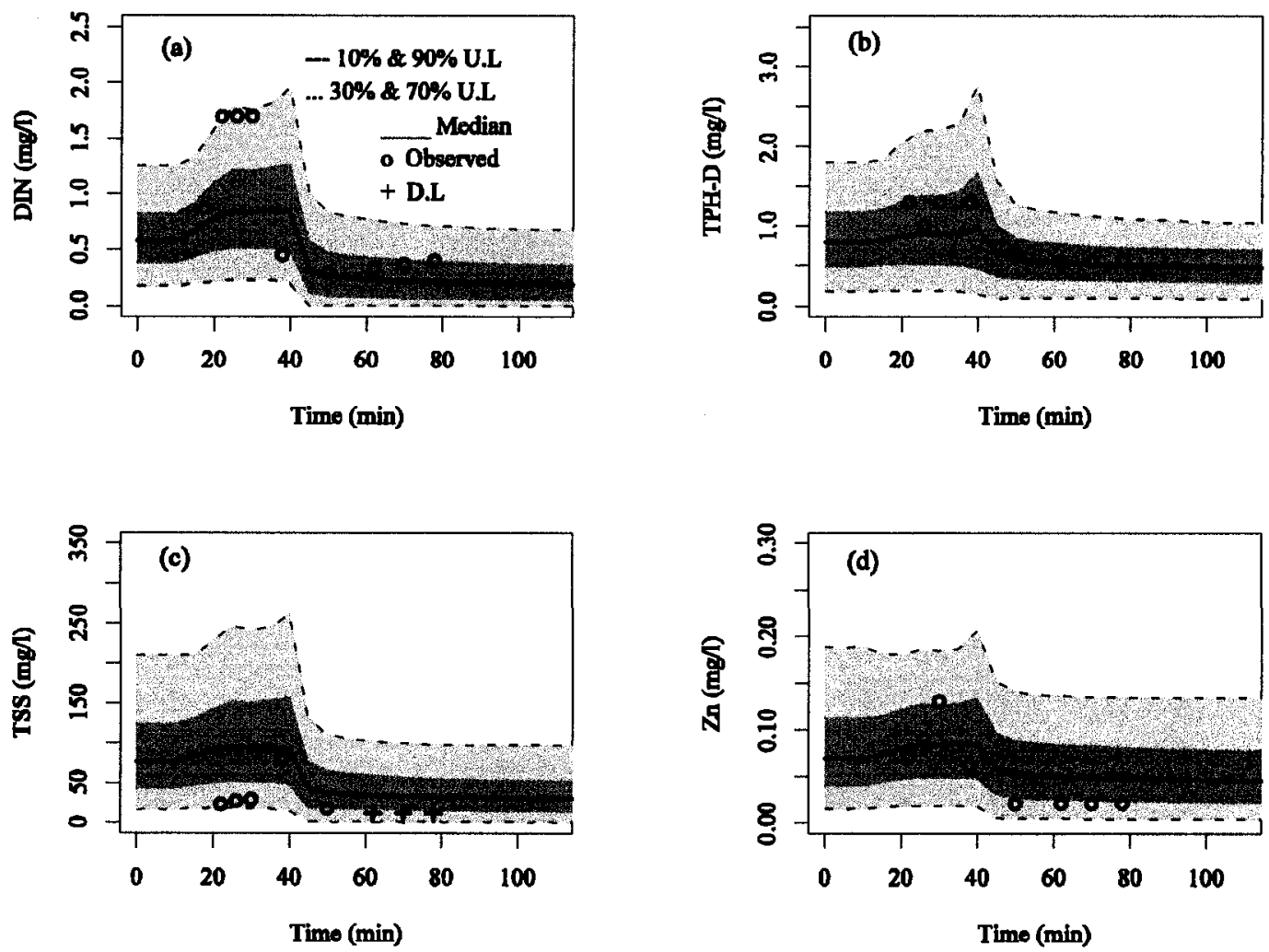

Figure 9 Monte Carlo simulations of predicted pollutant concentrations at the catchment's outlet for the 07/22/2006 storm event. U.L: Uncertainty Limits. The dark shaded region indicates the $30 \%$ and $70 \%$ uncertainty limits. The light grey shaded region indicates the $10 \%$ and $90 \%$ uncertainty limits. Observed concentrations values and the reported detection limits were plotted as discrete points.

The total (observed and estimated) washed off mass was computed for the 06/21/2006 and 07/22/2006 storm events. The total observed mass was computed as the sum of the products between observed concentration and runoff volume over the 
number of samples collected during the storm. The total estimated mass was computed similarly but using the estimated concentrations (Figure 9 and Figure 9). Table 8 shows results for each contaminant and storm event. The three estimated values correspond to the $30 \%\left(q_{30}\right), 50 \%\left(q_{50}\right)$, and $70 \%\left(q_{70}\right)$ uncertainty limits. For each validation exercise, the observed mass fell within the $30 \%$ and $70 \%$ uncertainty limits obtained from the model. However, some median estimated values were higher than those observed, which suggests that the model overestimated the total observed washed off mass.

Table 8 Observed and estimated total mass for the storms used for validation.

\begin{tabular}{cccccc}
\hline \multirow{2}{*}{ Storm } & \multirow{2}{*}{ Pollutant } & \multicolumn{5}{c}{ Total Mass $(\mathrm{g})$} \\
\cline { 3 - 6 } $6 / 21 / 2006$ & Observed & Estimated $\left(q_{30}\right)$ & Estimated $\left(q_{50}\right)$ & Estimated $\left(q_{70}\right)$ \\
\hline & TSS & 5259 & 2548 & 5354 & 9378 \\
& TPH-D & 39 & 32 & 60 & 100 \\
& Zn & 4.0 & 3.3 & 6.4 & 10.6 \\
& DIN & 34 & 23 & 44 & 71 \\
& TSS & 4887 & 3543 & 6858 & 11249 \\
& TPH-D & 97 & 40 & 71 & 112 \\
& Zn & 4.5 & 3.7 & 6.7 & 10.3 \\
& DIN & 41 & 33 & 59 & 90 \\
\hline
\end{tabular}

\section{Conclusions}

An accumulation and wash-off model for common stormwater pollutants was calibrated for a standard parking lot using TSS, TPH-D, DIN, and Zn measured in runoff samples. Parameter values were found for each storm event assuming a null residual mass approach. A range of parameter values was provided for each parameter of the model (Figure 6). In general, a normal probability distribution was sufficient to describe 
the variability of the optimized parameter values. The ranges of parameter values for TSS were higher than those for $\mathrm{Zn}$, DIN, and TPH-D. This is possibly due to the fact that TSS concentrations are higher in magnitude when compared to the other pollutants. This may be avoided by normalizing the concentration values before running the model.

The transport of contaminants due to runoff is a complex hydrological, physical and chemical phenomenon that depends on factors such as the catchment's response to rainfall, land use, wind, and human activities. The four-parameter model studied here reproduced reasonably well the dynamics of pollutant transport during the runoff process. Usually a 3 to 5 parameter model is recommended for rainfall-runoff modeling (Jakeman and Hornberger, 1993). Even though the wash-off model follows an exponential decay trend, the non-linear nature of the problem was taken into account and reflected in the range of parameter values of the wash-off exponent (w).

The aim of this work was to apply a wash-off model on a variety of storm events and to provide scientists, watershed managers, regulators and planners with a range of parameter values for modeling purposes. The employed wash-off model has been extensively used to estimate TSS concentrations, but little documentation is found for $\mathrm{Zn}, \mathrm{TPH}-\mathrm{D}$, and DIN. However, these model results should be considered as sitespecific and care must be taken when extending its usage to other watersheds.

Concentration values were drawn from a fitted probability distribution when a BDL value was reported. A gamma probability distribution seemed to represent concentration values above the reporting limit. In general, BDL values were reported at the decreasing (falling limb) portion of the hydrograph so that the total washed off mass was not significantly affected. However, a more detailed analysis on how this approach affects the final results is recommended.

The SA algorithm was successfully used as an optimization technique. An annealing schedule was determined for the characteristics of the objective function. Computation 
time was not a concern and the algorithm converged quickly for the majority of the cases.

The statistical information provided in this study is valuable for conducting risk analysis studies and estimating water quality impacts due to stormwater. Parameter values obtained in this study could be used in more complex applications such as Bayesian based models where an apriori probability distribution is necessary. 


\title{
CHAPTER 3
}

\section{MODELING URBAN STORMWATER QUALITY TREATMENT: MODEL DEVELOPMENT AND APPLICATION TO A SURFACE SAND FILTER}

\begin{abstract}
A statistical and mathematical model for simulating contaminant removal from a surface sand filter is introduced. The model is based on the mass balance principle and the assumption that an n-order treatment model describes the complex processes of pollutant removal. The parameters of the model are the removal rate $\mathrm{k}$ and the decay order $\mathrm{n}$. The model is deterministic: changes in space are not considered, and time variability of flow and influent contaminant concentration are taken into account. System field monitoring was performed between 2004 and 2006. A total of 17 storms were selected for the study. Runoff constituent analyses included: total suspended solids (TSS), total petroleum hydrocarbons - diesel range hydrocarbons (TPH-D), dissolved inorganic nitrogen (DIN, comprised of nitrate, nitrite, and ammonia), and zinc ( $\mathrm{Zn}$ ). The objective was to explore the capabilities of a two parameter removal model for predicting effluent pollutant concentrations. A gamma distribution was fitted to the optimized removal rate values and then a Monte Carlo simulation was performed to validate the calibration process. It was found that a second order approximation was most likely to describe the pollutant removal of TSS and TPH-D, and a first order approximation for $\mathrm{Zn}$. Poor model performance was obtained for DIN. Several examples are shown in order to illustrate the methodology and the application of the model.
\end{abstract}




\section{Introduction}

Our awareness of stormwater impacts has led us to the development of different stormwater treatment strategies. Previous knowledge regarding traditional water treatment systems (drinking and wastewater) and the evaluation of current stormwater treatment strategies has helped designers understand what is appropriate to mitigate the deleterious effects of stormwater. The final selection of site specific stormwater treatment is driven by: the quantity of water to treat; pollutants to remove; peak flow; treatment efficiency; regulatory constraints; cost; and other design factors (Minton, 2002). During the last decade, stormwater has been considered the next environmental challenge to be addressed (EPA, 1996).

The surface sand filter is one of the stormwater treatment measures recommended for stormwater mitigation, yet not commonly selected. The system appears in state stormwater design manuals and its performance has been documented (Roseen et al, 2006; Minton, 2002; EPA, 1999). It has been shown that sand filters are able to achieve high removal efficiencies for sediments and biochemical oxygen demand (BOD) when the system is properly maintained (EPA, 1999). Total metal removal is moderate and nutrient removal is often low.

Although the sand filter's performance has been reported in numerous studies, there is little research investigating the usage of mathematical models to describe the stormwater quality improvement from sand filters. Managers, regulators, engineers, and scientists need tools to simulate the performance of sand filters under a variety of hydraulic and hydrological conditions. Moreover, efforts are needed to undertake the problem of predicting effluent concentrations.

Changes in concentration as a function of time can be described by kinetic processes (Capellos and Bielski, 1972). This mathematical model describes the decrease of 
concentration with time as a function of a removal rate $(k)$ and the current concentration value to the $\mathrm{n}$ power (the reaction order). The reaction order may be an integer or a real number. Experience from wastewater treatment indicates that the reaction rate is a function of temperature and the nature of the chemical, biological, and physical processes that drive the reaction for each chemical or constituent. A chemical reaction is of zero-order if it is independent of the substance concentration; in other words, the amount $\mathrm{dC}$ by which the substance decreases in $\mathrm{dt}$ is constant throughout the duration of the reaction. In cases where $n \geq 1$, changes in concentration occur rapidly (proportionally to the $\mathrm{n}^{\text {th }}$ power of $\mathrm{C}$ ) and slowly approach zero. The reaction order is likely to be greater than 1 in stormwater due to the fact that the initial pollutant concentrations are commonly comparatively low (Minton, 2002). The reaction order has not been determined yet in stormwater treatment systems for a variety of pollutants (Minton, 2002).

Removal of contaminants from granular filters has been studied by using an exponential decay function. Iwasaki (1937) proposed an exponential decay model in which the decrease of concentration with media depth is proportional to a filtration coefficient multiplied by the current concentration value. The removed suspended particles accumulate in the filter pores. Iwasaki's model also provides a mathematical expression for determining the accumulating deposit in the filter medium. Changes in the filtration coefficient are expected over time due to the accumulation of suspended particles in the filter media. Iwasaki's model has been used in combination with other physicochemical and biological models to simulate the dynamics of sand filters in drinking water applications (Campos et al., 2006).

Some studies have assumed a first order decay model to describe treatment by stormwater measures (Wong et al., 2006; Wang et al., 2004). However, usually the following assumptions are considered: steady state flow, constant influent 
concentrations, and invariability of the model parameters. Kadlec (2000) studied the adequacy of first order decay models for the design of treatment wetlands. The parameters of the first order decay model were a removal rate $k$ and a background concentration $C^{*}$. This study recommended the use of a spectrum of parameter values for modeling applications. Further discussion of the so-called $\mathrm{k}-\mathrm{C}^{\star}$ model can be found in the literature (e.g, Wong, et al., 2006; Kadlec and Knight, 1996).

A surface sand filter was monitored for stormwater quality at a parking lot in Durham, $\mathrm{NH}$. This study explored the applicability of an n-order decay model to estimate effluent pollutant concentrations from a surface sand filter. The parameters of the model are: the removal rate $\mathrm{k}$ and the decay order $\mathrm{n}$. Particularly, the model was explored using a zero, a first, and a second order approximation $(n=0,1,2)$. Although the model was not developed to describe in detail complex chemical, physical, and biological processes; it was conceptualized with the intention of emulating the decay function observed in other science fields such as chemical kinetics and sand filtration. Calibration of the mathematical model is presented and optimum parameter values are provided. This study provides appropriate probability distribution functions (PDFs) for the removal rate parameter and identifies the most likely decay order for the contaminants of concern. The model's capability for predicting effluent concentrattons is tested by performing Monte Carlo simulations. Additionally, the distribution of the estimated effluent Event Mean Concentrations (EMC) was compared against the distribution of the observed effluent EMCs. An Event Mean Concentration (EMC) is a parameter commonly used to characterize pollutant concentrations of a storm event (Sansalone and Chad, 2004; Lee and Bang, 2000). Examples for different storm events are provided. 


\section{Surface Sand Filter}

The surface sand filter monitored for this research is located at the University of New Hampshire Stormwater Center in Durham, NH. A commuter parking lot provides the stormwater runoff to the filter. The treatment system is a Low Impact Development (LID) design comprised of a sedimentation forebay and filter basin. The filter bed is $2.4 \mathrm{~m} \mathrm{x}$ $6.1 \mathrm{~m}$. The filter is $0.6 \mathrm{~m}$ high and uses a mix of coarse to medium grain sand with $D_{10}=$ $0.3 \mathrm{~mm}, D_{50}=0.7 \mathrm{~mm}$, and $D_{85}=2 \mathrm{~mm}$. The sedimentation forebay helps prevent the filter from premature clogging by removing the largest particles and performing flow equalization. The forebay was designed to hold $25 \%$ of the water quality volume (WQV, $25 \mathrm{~mm}$ of precipitation on $4047 \mathrm{~m}^{2}$ of watershed). The designed WQV was $92.5 \mathrm{~m}^{3}$ and corresponds to the daily storm volume not exceeded $90 \%$ of the days with measurable precipitation. The filter basin above ground volume can hold the remaining $75 \%$ WQV. Temporary ponding is expected during larger storm events due to saturation of the filter media and the fact that inflow exceeds outflow. The filter bed is sub-drained by a $0.15 \mathrm{~m}$ diameter, perforated pipe bedded in a $0.20 \mathrm{~m}$ layer of crushed stone $\left(D_{50}=19 \mathrm{~mm}\right)$. Design parameters were adopted from the New York State Stormwater Management Design Manual (2001).

Physical settling of the largest particles occurs in the sedimentation forebay. Physical and chemical water quality treatment occurs in the filter basin. A sand filter is commonly viewed as a system for removing mostly suspended solids. However, it has been shown that sand filters have the ability to remove dissolved phosphorus and metals (Minton, 2002.). Performance of the surface sand filter used for this research is found in Roseen et al. (2006) and Ballestero et al. (2005). 


\section{Monitoring}

For this study, storm events were selected between August 2004 and September 2006 for a total of 17 discrete storm events. A storm was sampled if the total precipitation was higher than $2.5 \mathrm{~mm}$ and preceded by at least 72 hours of dry weather. Automated samplers (6712SR ISCO) were used to perform the sampling program. Although the ISCO samplers collected up to 24 samplers per storm event, normally only 8-12 samples were used to characterize both influent and effluent stormwater quality. The sampling program for the system was based on analyses of various effluent hydrographs. Precipitation, influent, and effluent flows were monitored, so a hydraulic model to estimate discharge was not necessary.

Constituent analysis of water samples included: total suspended solids (TSS), total petroleum hydrocarbons-diesel range hydrocarbons (TPH-D), dissolved inorganic nitrogen (DIN, comprised of nitrate, nitrite, and ammonia), and zinc ( $\mathrm{Zn}$ ). Stormwater samples were analyzed by a laboratory that is state-certified for drinking water and wastewater. Guidance documents on collecting environmental data and the site quality assurance project plan were followed to assure quality of the results (EPA, 2006). Characteristics of the storm events selected for this research are presented in Roseen et al. (2006).

\section{Model Structure}

\section{Stormwater Treatment Model}

A simple $n$-order decay model was used to describe pollutant removal from the sand filter. An expression for computing effluent concentrations can be derived from a water 
balance equation combined with the $n$-order decay model. This expression can be written as (Wang et al., 2004):

$$
\frac{d C_{\text {out }}}{d t}=\frac{C_{\text {in }} Q_{\text {in }}-C_{\text {out }} Q_{\text {out }}}{S}-\frac{C_{\text {out }}}{S} \frac{d S}{d t}-k C_{\text {out }}^{n}
$$

where $C_{\text {out }}$ represents the effluent concentration $(\mathrm{mg} / \mathrm{l}) ; \mathrm{C}_{\text {in }}$ is the influent concentration ( $\mathrm{mg} / \mathrm{l}) ; \mathrm{Q}_{\mathrm{in}}$ is the influent flow rate $\left(\mathrm{m}^{3} /\right.$ day); $Q_{\text {out }}$ is the effluent flow rate $\left(\mathrm{m}^{3} /\right.$ day); $S$ is the storage within the system $\left(\mathrm{m}^{3}\right)$; $d S / d t$ is the change in storage $\left(\mathrm{m}^{3} /\right.$ day); $t$ is time (days); $\mathrm{k}$ is the removal rate $\left(\left(\mathrm{mg} / \mathrm{l}^{-\mathrm{n}+1} /\right.\right.$ day $)$; and $\mathrm{n}$ is the decay order $(-)$. A boundary condition is defined as the concentration at $t=0$. These units will be used throughout the paper.

Equation (8) is a dynamic model that takes into account flow variations at the influent and effluent locations. The control volume included: the sedimentation forebay, the filter basin above ground, and the filter media. It is also assumed that the parameters $k$ and $n$ represent the combined effect of various pollutant removal mechanisms. Spatial variation of the variables is not considered. The effect of precipitation on the water budget was neglected since influent flow rates were much higher in magnitude. The model was applied during storm events so that evaporation was not considered. Ground water effects and other infiltration sources were also not included since the system was constructed in a clay soil.

An analytical solution of the differential equation (8) can be found for some integer $n$ values (Appendix A). For this research, the analytical solution was used and the analysis was performed by using a zero, a first, and a second order approximation $(n=0,1,2)$. For every storm the duration of the event was divided into time steps $\Delta t=5 \mathrm{~min}$. The final solution was obtained by systematically solving the analytical solution of (8) for 
each time interval. The effluent concentration found at interval $i$ was then used as initial concentration for the $i+1$ interval.

\section{The Objective Function}

An objective function was necessary to estimate the best set of model parameters $\Theta$ $=\{k, n\}$. The sum-of-squares estimator was adopted and is written as follows:

$$
O(\Theta)=\min \sum_{i=1}^{m}\left(C_{o u t, o b s}^{i}-C_{o u t, e s t}^{i}(\Theta)\right)^{2}
$$

where $O$ denotes the objective function; $\Theta$ represents the vector of parameter values; $\mathrm{C}_{\text {out,obs }}$ are the measured effluent concentrations; $C_{\text {out,est }}$ are the estimated effluent concentrations obtained from the treatment model (12); i denotes the current sample; and $m$ is the number of samples taken during the storm event.

\section{Optimization Technique}

In this study, the simulated annealing (SA) algorithm was used as the optimization technique to minimize the objective function (9). The method uses a stochastic approach to locate the parameter values that maximize or minimize the objective function. A new set of parameter values are chosen from a probability distribution that depends on the change of the objective function $(\Delta \mathrm{O})$ and a parameter $\mathrm{T}$ called the temperature (Kirkpatrick et al, 1983). This probability distribution is written as follows: 


$$
p(\theta)=e^{-\frac{\Delta O}{T}}
$$

The stochastic process is set up initially at a very high temperature so that new parameter values are highly likely to be accepted. Therefore, the parameters' space is searched extensively at the early stage of the process. The temperature is reduced during the process according to a "cooling schedule" chosen accordingly to the shape of the objective function. A solution that is not the "best" may be accepted occasionally so the algorithm does not get stuck in a local minimum.

\section{Measured Concentrations Below Detection Limit}

A detection limit is reported when pollutant concentrations are below the analytical reporting limit of the laboratory. Such concentrations are reported as being below the analytical detection limit (BDL). Half of the detection limit is commonly used if a discrete value is needed rather than a range (from 0 to $B D L$ ). Table 9 displays the percentages of influent and effluent concentration values reported as BDL for each contaminant, for the storms and samples used in this study. In this study, the detection limits were normally: $0.50 \mathrm{mg} / \mathrm{l}$ for TPH-D, $0.03 \mathrm{mg} / \mathrm{l}$ for $\mathrm{Zn}, 0.5 \mathrm{mg} / \mathrm{l}$ for DIN, and $10 \mathrm{mg} / \mathrm{l}$ for TSS.

Table 9 Summary of samples with concentrations reported as BDL.

\begin{tabular}{ccccccc}
\hline \multirow{2}{*}{ Location } & \multirow{2}{*}{ Format } & \multirow{2}{*}{$\begin{array}{c}\text { Total Number } \\
\text { of Samples }\end{array}$} & \multicolumn{4}{c}{ BDL Samples } \\
\cline { 4 - 7 } & & TPH-D & $\mathrm{Zn}$ & DIN & TSS \\
\hline \multirow{3}{*}{ Influent } & Number & 166 & 36 & 18 & 45 & 20 \\
& Percentage & $100 \%$ & $23 \%$ & $14 \%$ & $34 \%$ & $8 \%$ \\
& Number & 132 & 97 & 61 & 27 & 37 \\
& Percentage & $100 \%$ & $69 \%$ & $46 \%$ & $20 \%$ & $21 \%$ \\
\hline
\end{tabular}


Estimates of the BDL concentration values can be made by implementing a probabilistic approach. In this scenario, a probability distribution is fitted to the data using only concentration values above the reported detection limit. Then, concentration values between zero and the detection limit can be drawn from the fitted probability distribution (Helsel and Hirsch, 2002). Note that the accuracy of this method depends on the amount of data available above detection limit.

For this study, a Gamma distribution was fitted to the data. The parameters $\gamma$ (shape) and $\theta$ (scale) of the distribution were computed using the Maximum Likelihood Estimator. The parameter values of the fitted probability distribution are shown in Table 10 . The Kolmogorov-Smirnov test (KS-test) was used to assess whether or not the data followed the fitted distribution. The D statistic was computed for a level of significance $\alpha=0.05$ (95\% confidence interval) and $i$ concentration values above the detection limit. The data

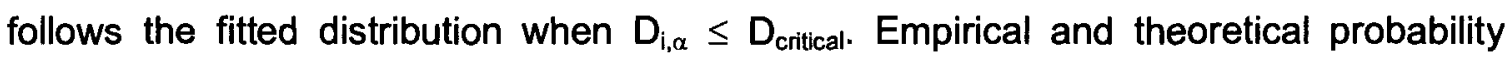
distributions functions (PDFs) are shown in Figure 10. The D statistic represents the maximum vertical deviation between the empirical and theoretical data probability distributions. This methodology was used to develop probability distributions at the influent (Avellaneda et al., 2008)

Table 10 Parameters of the gamma distributions fitted to the effluent data.

\begin{tabular}{cccccc}
\hline Contaminant & $\begin{array}{c}\text { Total samples } \\
\text { above DL (i) }\end{array}$ & $\gamma$ (shape) & $\theta$ (scale) & $\mathrm{D}_{\text {critical }}$ & $\mathrm{D}_{\mathrm{i}, \alpha}$ \\
\hline TPH-D & 35 & 4.61 & 0.15 & 0.23 & 0.13 \\
$\mathrm{Zn}$ & 71 & 2.76 & 0.01 & 0.16 & 0.17 \\
DIN & 105 & 1.72 & 0.46 & 0.13 & 0.07 \\
TSS & 95 & 2.12 & 16.67 & 0.14 & 0.07 \\
\hline
\end{tabular}

Level of significance $\alpha=0.05$ 

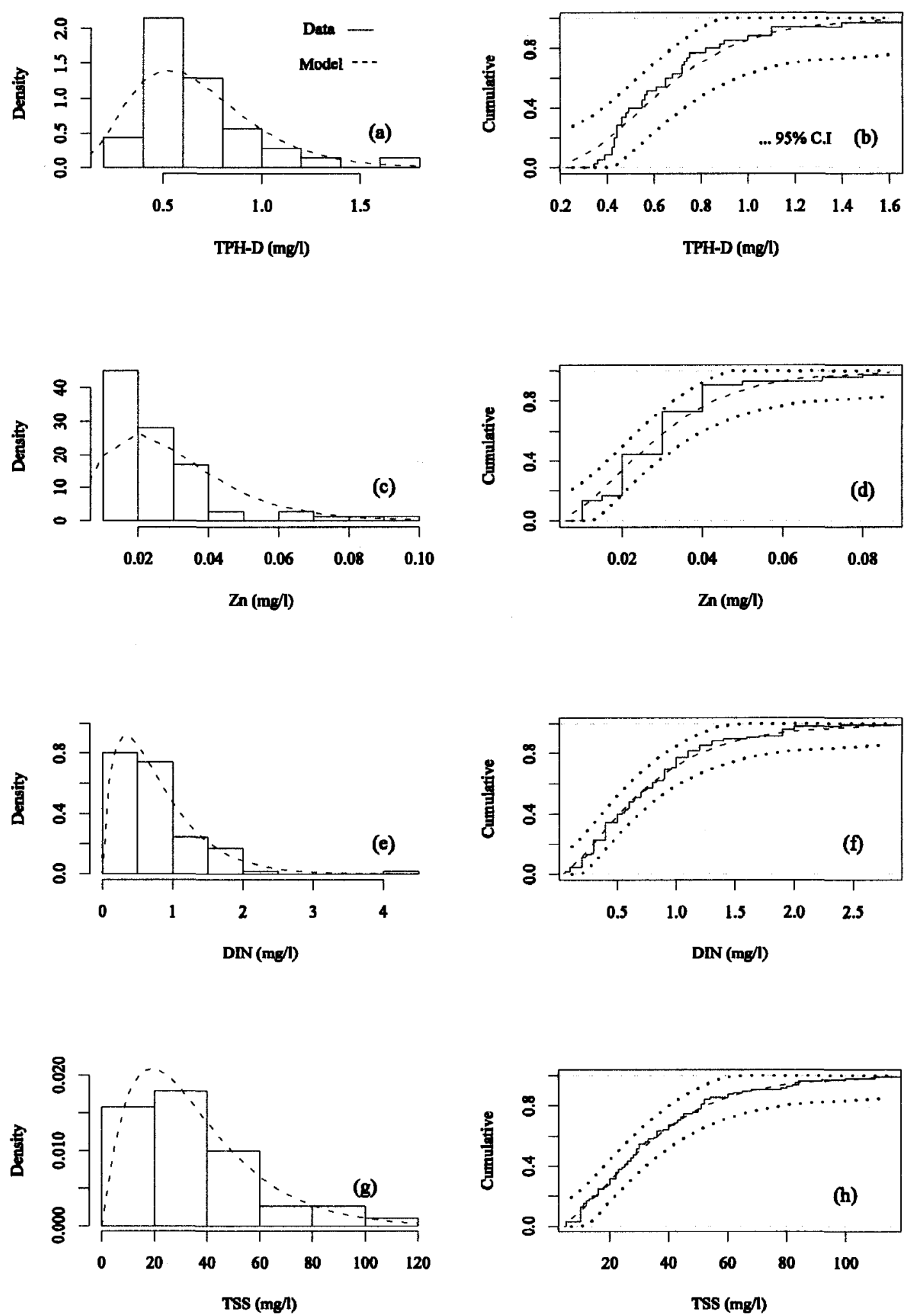

Figure 10 Empirical (Data) and theoretical data (Model) probability distributions for sand filter effluent. C.I: Confidence Interval. 


\section{Calibration Procedure}

Optimal parameter values for the removal rates $(k)$ and the decay orders $(n)$ were obtained on a storm by storm basis. A total of 15 storms were utilized for calibration purposes, and two storms were selected for model validation using the Monte Carlo method. A continuous simulation approach was not performed because inter-event samples were not available. The initial concentration (at the beginning of the storm) was assumed to be zero. This assumption was considered reasonable since at the start of these of storms only groundwater baseflows existed typically at detection level concentrations for the contaminants of the study.

During the optimization process new $k$ and $n$ candidates were randomly generated according to the SA algorithm. The root mean square error (RMSE) was computed for each set of candidates. For the new candidates to be generated it was necessary to establish a lower and upper limit of the parameters. A lower limit of 0 and an upper limit of 100 were used initially to test what the parameter space would be. The $n$ value was selected among a first, second, and third order approximation $(n=0,1,2)$.

A linear decrement function was adopted to reduce the temperature $T$ of the SA algorithm. The "cooling" schedule was defined by

$$
T_{t+1}=a T_{t} \quad t=0,1, \ldots, s_{t}
$$

where $T$ is the temperature; $s_{t}$ is the number of steps of the cooling schedule; and $a$ is the temperature reduction coefficient that varied between 0 and 1 (Hopgood, 2000). For this research, these parameters were set to $T=10,000, s_{t}=20, a=0.9$, and 20,000 
iterations were used. These previous parameter values were found by initially re-running the model several times and verifying that the random walk searched the entire domain and the temperature did not reduce too quickly.

\section{Results}

\section{Model Application}

Figures 11 and 12 show model results for some of the storms considered in this study. Influent and effluent monitored hydrographs are also plotted. Influent pollutographs for all the contaminants of study were obtained from a calibrated accumulation and wash-off model (Avellaneda et al., 2008). Estimated effluent pollutant concentrations are plotted along with the observed effluent concentrations. RMSE values are also reported.

The influent hydrograph for the 03/08/2005 event increased gradually, reached a maximum peak flow, and then decayed (Figure 11(a)). A delay was clearly observed in the effluent hydrograph. This is due to the sand filter's storage capacity and infiltration rate; although, it also depends on factors such as rainfall duration and intensity. An influent peak flow reduction was also observed. For all the contaminants, the first observed effluent concentration value was reported as BDL. The model does not explain the reported lower concentration of the first sample and tends to follow the trend of the subsequent higher concentration values. In this study, observed concentration values were used as they were reported from the laboratory, but not in the model itself since they could not be incorporated directly, but rather through their transformation by the real 
time model. In general, all the effluent pollutographs showed a smooth exponential decay of concentration over time.
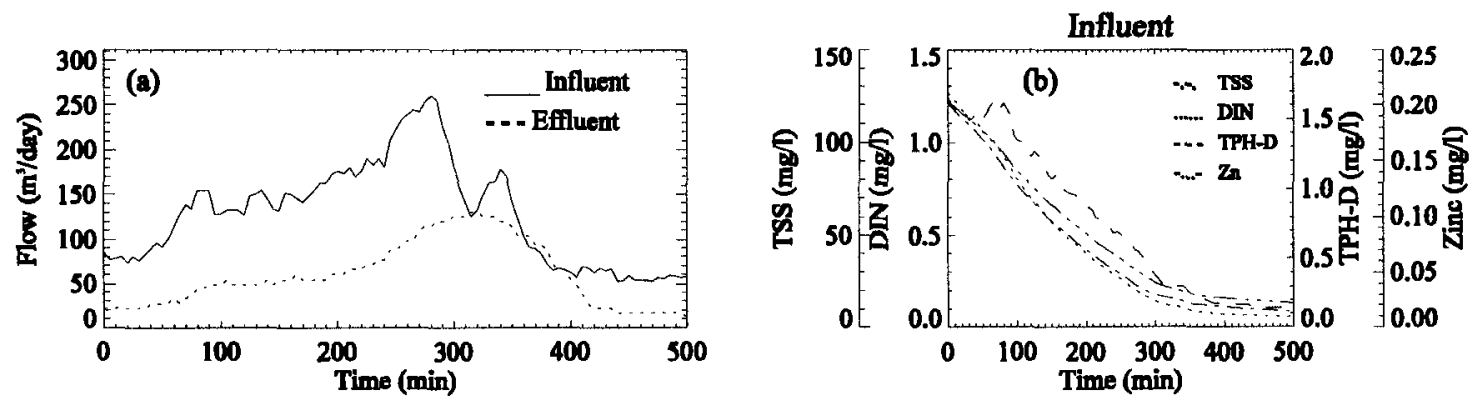

Effluent
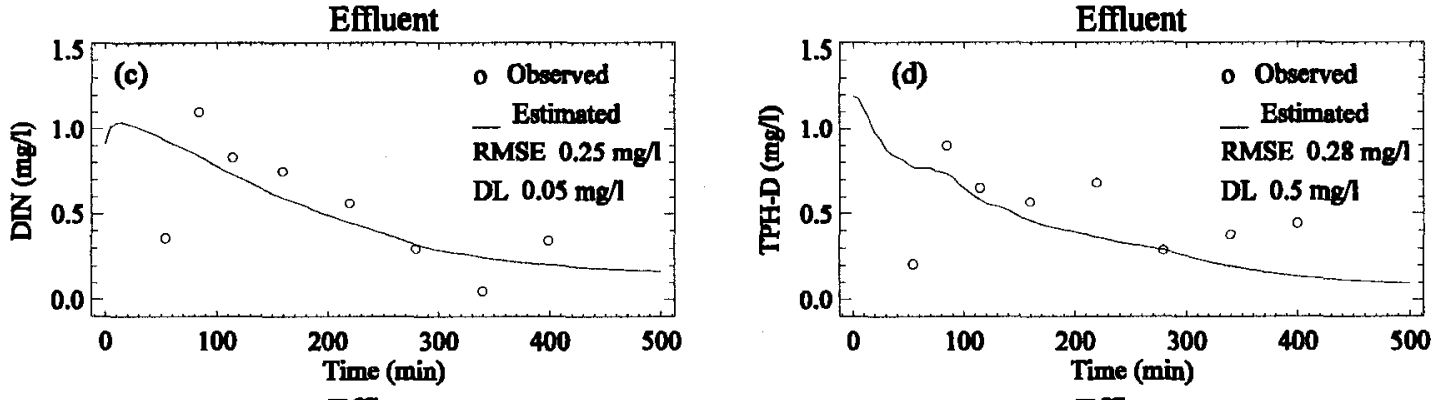

Effluent
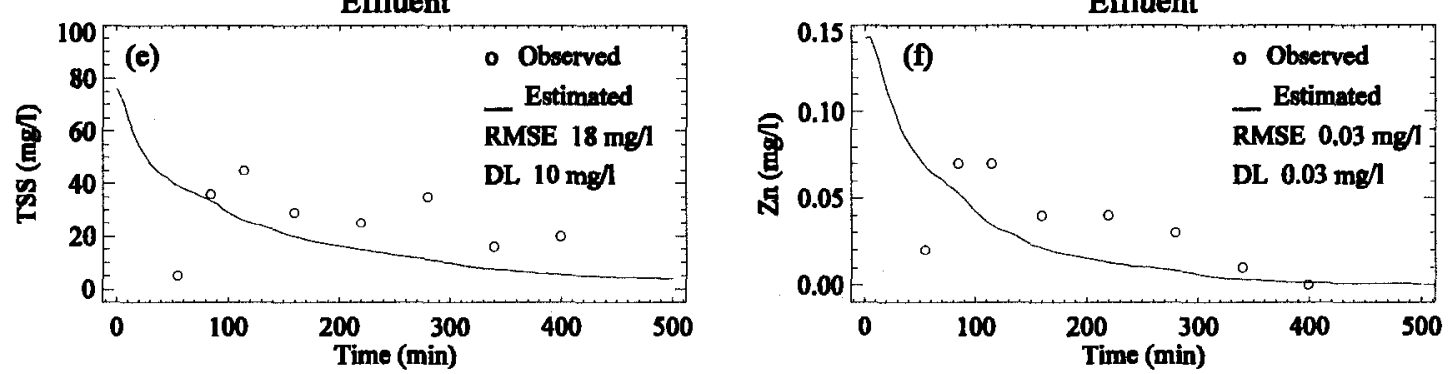

Figure 11 Results for the 03/08/2005 storm. Optimized parameter values are shown in Table 11

Figure 12 shows model result for the 04/20/2005 storm event. Multiple flow peaks were observed on the influent hydrograph (Figure 12(a)). As expected, the influent hydrograph is attenuated by the storage available in this stormwater system, producing a smooth effluent hydrograph. The last two concentration values were reported below the detection limit for DIN, the last three for $\mathrm{Zn}$, the last one for TSS, and only the first two 
sample concentrations were reported above the detection limit for TPH-D. The model described the general trend observed of the measured concentrations.
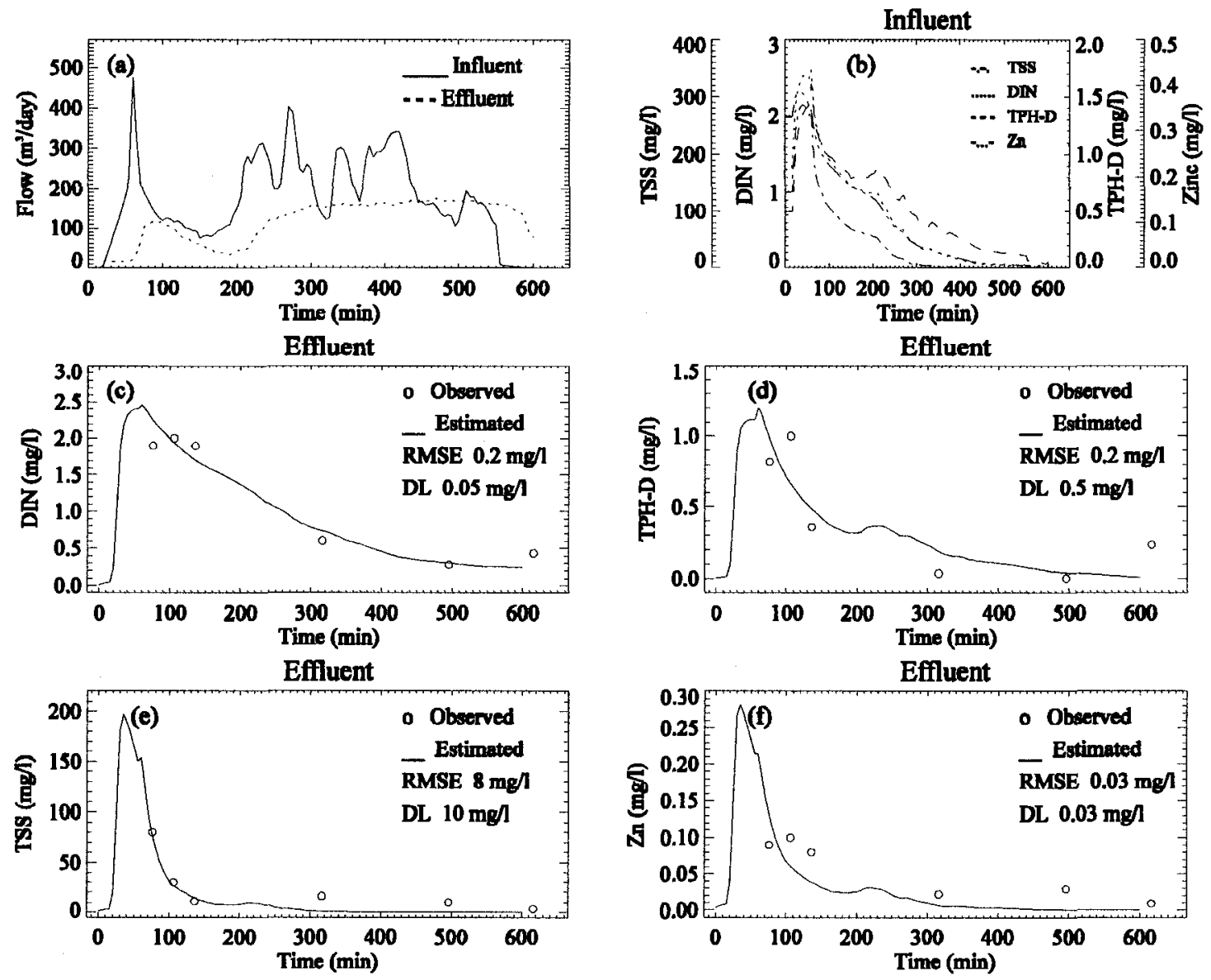

Figure 12 Results for the 04/20/2005 storm. Optimized parameter values are shown in Table 11.

The optimized parameter values for all the storms and contaminants included in this study are presented in Table 11. RMSE values are reported. Figure 13 shows box and whisker plots for the removal rate optimized parameter values. The highest removal rates were obtained for $\mathrm{Zn}$ and TPH-D; lower removal rates were achieved for TSS; and values relatively close to zero for DIN. 
Table 11 Optimized removal rates $(k)$ and decay orders $(n)$ for different storms and contaminants

\begin{tabular}{|c|c|c|c|c|c|c|c|c|c|c|c|c|}
\hline \multirow{2}{*}{$\begin{array}{c}\text { Rainfall } \\
\text { Event } \mathrm{m} / \mathrm{d} / \mathrm{y}\end{array}$} & \multicolumn{3}{|c|}{ DIN } & \multicolumn{3}{|c|}{ TPH-D } & \multicolumn{3}{|c|}{ TSS } & \multicolumn{3}{|c|}{ ZINC } \\
\hline & $k$ & $n$ & RMSE & $k$ & $n$ & RMSE & $k$ & $n$ & RMSE & $k$ & $n$ & RMSE \\
\hline $09 / 18 / 04$ & 0.4 & 0 & 0.10 & 15.0 & 1 & 0.11 & 0.0 & 0 & 48 & 13.1 & 1 & 0.01 \\
\hline $10 / 30 / 04$ & 0.0 & 2 & 0.14 & 38.6 & 2 & 0.09 & 0.0 & 0 & 16 & 59.5 & 2 & 0.01 \\
\hline $01 / 14 / 05$ & 0.0 & 2 & 0.72 & - & - & - & 31.0 & 2 & 34 & - & - & - \\
\hline $02 / 10 / 05$ & 3.0 & 2 & 0.28 & 6.8 & 2 & 0.20 & - & - & - & 91.9 & 1 & 0.03 \\
\hline 03/08/05 & 6.8 & 2 & 0.25 & 57.2 & 2 & 0.27 & 1.6 & 2 & 18 & 75.2 & 1 & 0.03 \\
\hline $03 / 28 / 05$ & 3.1 & 2 & 0.21 & 0.0 & 2 & 0.32 & 5.7 & 2 & 47 & 18.9 & 1 & 0.01 \\
\hline $04 / 20 / 05$ & 0.7 & 2 & 0.19 & 57.2 & 2 & 0.21 & 85.8 & 1 & 8 & 74.0 & 1 & 0.03 \\
\hline $08 / 13 / 05$ & 0.0 & 2 & 1.27 & 8.4 & 2 & 0.43 & 18.2 & 1 & 9 & - & - & - \\
\hline $09 / 15 / 05$ & - & - & - & 51.3 & 2 & 0.15 & 0.0 & 0 & 32 & 17.9 & 1 & 0.00 \\
\hline $11 / 06 / 05$ & - & - & - & - & - & - & - & - & - & 50.7 & 1 & 0.01 \\
\hline $11 / 30 / 05$ & 0.0 & 1 & 0.35 & 23.3 & 2 & 0.18 & - & - & - & 79.7 & 1 & 0.01 \\
\hline $12 / 16 / 05$ & 0.6 & 1 & 0.12 & - & - & - & 1.5 & 2 & 12 & 125.0 & 1 & 0.02 \\
\hline $01 / 11 / 06$ & 0.0 & 2 & 0.55 & 18.5 & 2 & 0.16 & 0.0 & 0 & 30 & 0.0 & 2 & 0.01 \\
\hline $02 / 17 / 06$ & 0.0 & 0 & 0.24 & 36.7 & 2 & 0.21 & 2.0 & 2 & 10 & 111.7 & 1 & 0.02 \\
\hline $03 / 13 / 06$ & 0.0 & 0 & 0.31 & - & - & - & 0.5 & 2 & 12 & 62.8 & 1 & 0.01 \\
\hline
\end{tabular}

Note: "-" Indicates that data are not available. $k$ has units of $(\mathrm{mg} / \mathrm{l})^{-\mathrm{n}+1} /$ day. $n$ is dimensionless.

RMSE has units of $\mathrm{mg} /$. 
DIN

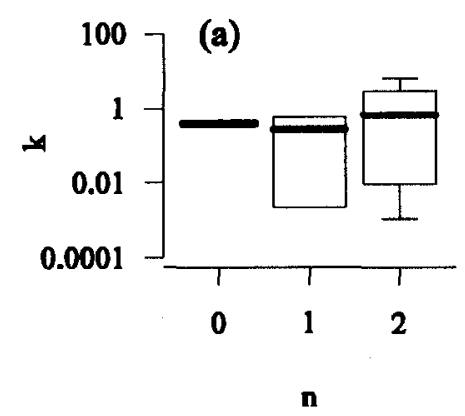

TSS

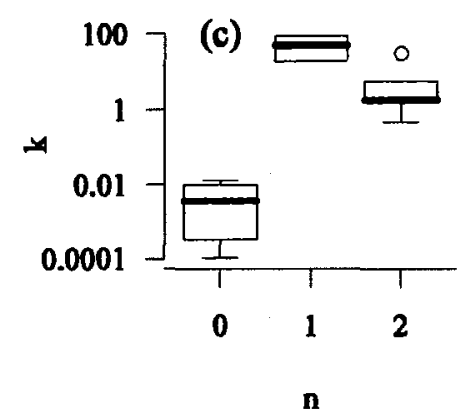

TPH-D

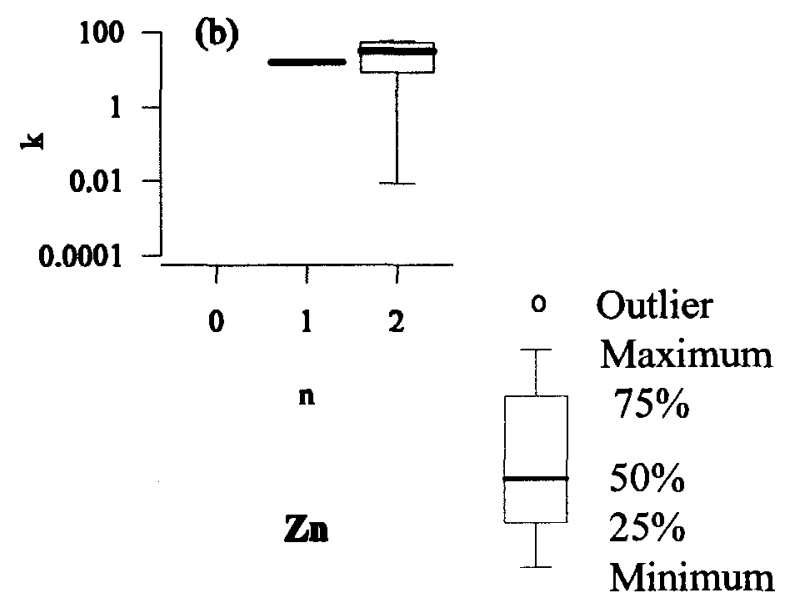

Figure 13 Box and whisker plot of optimized removal rates (k) for all the contaminants and decay order models $(n=0,1,2)$. $k$ has units of $(m g / l)^{-n+1} /$ day.

\section{Statistical Analysis}

The cumulative distribution function (CDF) was developed for the optimized removal rate parameter values (Figure 14). Afterward, different probability distributions were fitted to the optimized parameter values and tested. It was found that the gamma distribution was appropriate to represent the CDFs obtained from the optimized values. Table 12 shows the gamma distribution parameters fitted to the data. The parameters of the gamma distribution are the shape $\gamma$ and the scale $\theta$. These two parameters are related to 
the mean $\bar{x}$ and the variance $\mathrm{s}^{2}$ of the data as follows: $\bar{x}=\gamma \theta$ and $\mathrm{s}^{2}=\gamma \theta^{2}$. The KStest was used to measure the goodness-of-fit of the fitted gamma distributions. A $95 \%$ confidence interval is displayed in Figure 14 as well.

Table 13 shows the frequency of the optimized decay orders for the different contaminants. In this case, a probability function was not fitted since the main goal was to explore what $n$ values were more likely to characterize the treatment capabilities of the system. Results indicate that $n=2$ was the optimum observed most frequently for TSS and DIN (Table 13). However, for some storms, $n=1$ and $n=0$ were found to be the best parameter values. For TPH-D, the treatment process seems to follow a second order decay model. A first order decay model is more likely to describe the removal of Zn.

Table 12 Parameters of the gamma distributions fitted to removal rate optimized values.

\begin{tabular}{cccccc}
\hline Contaminant & $\begin{array}{c}\text { Total number } \\
\text { of storms (j) }\end{array}$ & $\gamma$ (shape) & $\theta$ (scale) & $D_{\text {critical }}$ & $D_{j, \alpha}$ \\
\hline TPH-D & 11 & 1.9 & 15.2 & 0.39 & 0.18 \\
Zn & 13 & 2.4 & 25.1 & 0.36 & 0.21 \\
DIN & 13 & 0.3 & 3.7 & 0.36 & 0.33 \\
TSS & 12 & 0.2 & 51.6 & 0.38 & 0.18 \\
\hline
\end{tabular}

Level of significance $\alpha=0.05$ 
Table 13 Frequency of optimized decay orders for the different contaminants.

\begin{tabular}{cccc}
\hline \multirow{2}{*}{ Contaminant } & \multicolumn{3}{c}{ Decay Order $(\mathrm{n})$} \\
\cline { 2 - 4 } \cline { 4 - 4 } & $\mathrm{n}=0$ & $\mathrm{n}=1$ & $\mathrm{n}=2$ \\
\hline DIN & 3 & 2 & 8 \\
TPH-D & 0 & 1 & 10 \\
TSS & 4 & 2 & 6 \\
Zn & 0 & 11 & 2 \\
\hline
\end{tabular}

TSS

TPH-D
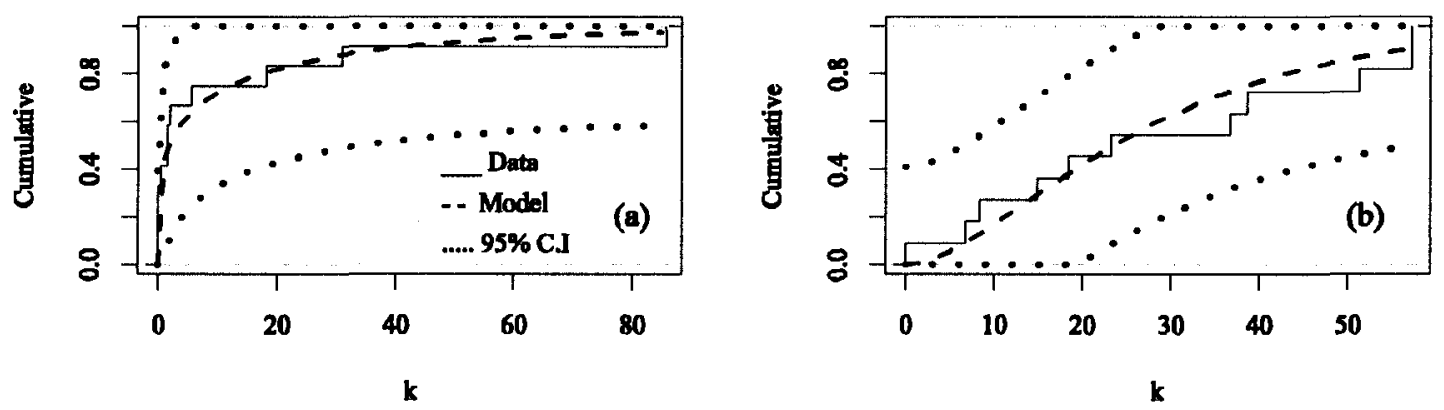

Zn

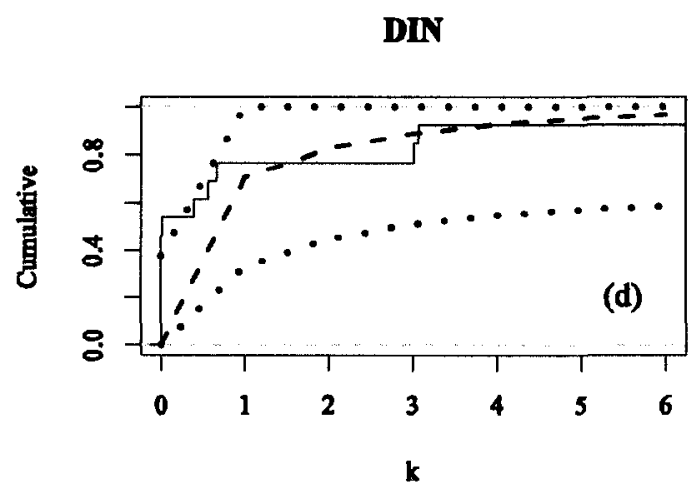

Figure $14 \mathrm{CDFs}$ of optimized removal rates (Data) and fitted gamma distributions (Model). A 95\% confidence interval (C.I) is shown for each contaminant. 


\section{Event Mean Concentration (EMC)}

For this study, the estimated effluent EMC was compared against the observed effluent EMC to evaluate the performance of the model. The EMC of a storm event is defined as the total pollutant load divided by the total runoff volume. Equation (12) can be used to compute the EMC when discrete samples are collected during the duration of the storm:

$$
E M C=\frac{\sum_{i=1}^{m} Q_{i} C_{i} \Delta t_{i}}{\sum_{i=1}^{m} Q_{i} \Delta t_{i}}
$$

where EMC is the effluent Event Mean Concentration (mg/l); $Q_{i}$ and $C_{i}$ are the average flows $\left(\mathrm{m}^{3} / \mathrm{day}\right)$ and concentrations $(\mathrm{mg} / \mathrm{l})$ within the time interval $\Delta \mathrm{t}_{\mathrm{i}}$ (day); and $\mathrm{m}$ is the total number of time intervals. EMCs have also been utilized to evaluate the performance of stormwater treatment measures (Barret, 2005).

EMCs were computed for the storms used for calibration. Figure 15 shows cumulative distribution functions for the observed effluent EMCs (Out (obs)) and the estimated effluent EMCs (Out (est)). Observed effluent EMCs were computed using the samples collected during the storm event. Estimated effluent EMCs were computed using the estimated pollutographs obtained after calibration and the respective effluent hydrograph. Note that the estimated pollutograph was computed using the calibrated parameters for each storm. 
DIN

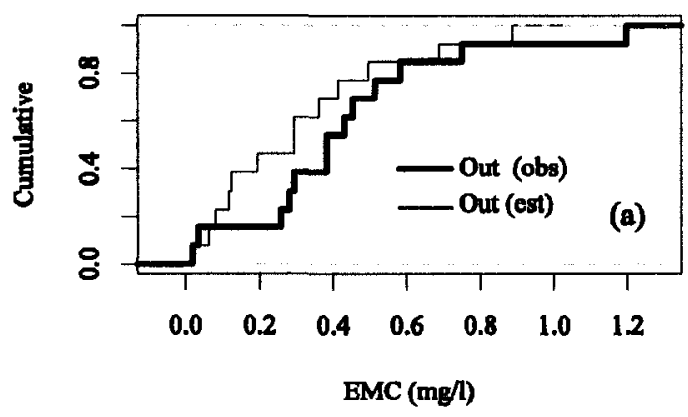

TSS

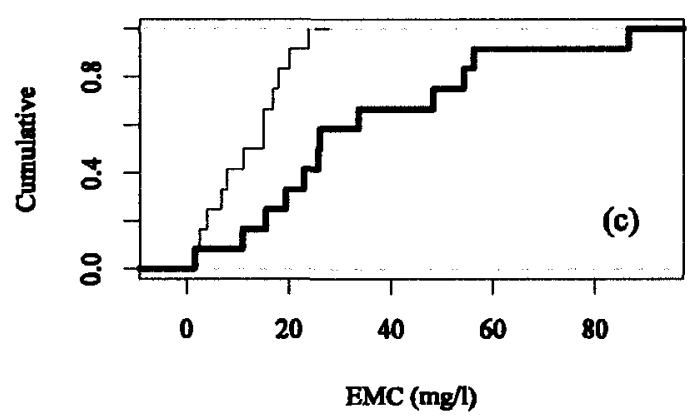

TPH-D

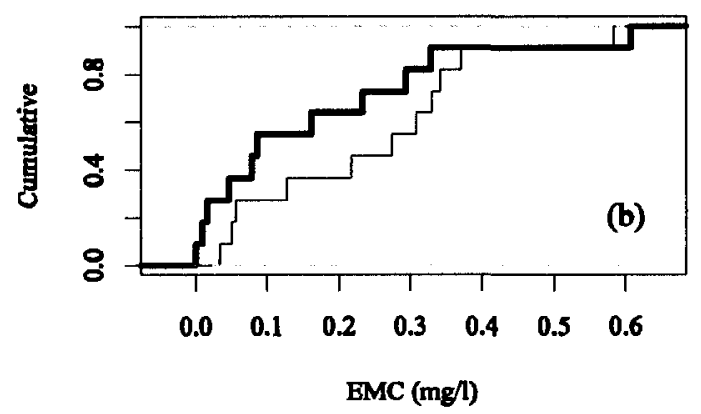

Zn

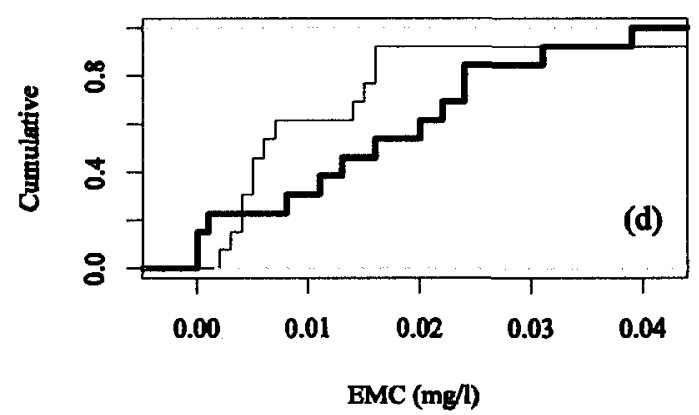

Figure 15 Sand filter CDF of Event Mean Concentrations (EMC). Out: effluent, obs: observed, and est: estimated.

The K-S test was performed to assess whether or not the estimated effluent CDF followed the distribution of the observed effluent CDF. The estimated effluent CDF for TSS did not pass the K-S test since $D_{j, \alpha}>D_{\text {critical }}$ for a level of significance $\alpha=0.05\left(D_{j, \alpha}=\right.$ 0.58, $\mathrm{D}_{\text {critical }}=0.37, \mathrm{j}=12$ storms). The model underpredicted effluent TSS concentrations. For DIN, TPH-D, and Zn, the estimated effluent CDFs passed the K-S test, which suggests that the calibrated model preserved the distribution of the observed effluent EMCs. 


\section{Monte Carlo Simulation}

A single set of parameter values should not be used for modeling purposes. As it has been reported so far in this study, the removal rate parameter $(k)$ and the decay order (n) vary among storms and contaminants. One could determine the PDF of the model parameters when data are available. When the parameters' PDFs are provided, then a Monte Carlo simulation can be performed to assess the prediction capabilities of the model. Monte Carlo simulations incorporate uncertainty into the analysis and it has been recommended as a useful tool when assessing the water quality characteristics that would result from different environmental scenarios (Beck, 1987; Walker, 1994).

For validation purposes, results of a Monte Carlo simulation are provided for two storm events: 05/02/2006 and 05/09/2006. The fitted gamma distributions (Table 12 and Figure 14) were used to generate (k) values. A decay order was selected for each contaminant: $n=2$ for TSS, TPH-D and DIN; and $n=1$ for $Z n$. These decay order values were selected upon the PDFs identified in Figure 14. The number of simulations was set to 5,000 for each contaminant.

Simulation results for the 05/02/2006 and 05/09/2006 storm events are shown in Figure 16 and Figure 17, respectively. The central solid line indicates the expected concentrations. The $10\left(q_{10}\right), 30\left(q_{30}\right), 70\left(q_{70}\right)$, and $90\left(q_{90}\right)$ percentiles were selected as a measure of uncertainty. For the 05/02/2006 storm, DIN and TSS observed effluent concentrations fell above the $90 \%$ uncertainty limit (Figure 16(a) and Figure 16(c)). This indicates that, for this storm, the model underestimated effluent pollutant concentrations for DIN and TSS. Note that uncertainty limits for DIN were almost identical, which indicates poor model performance. The model predicted some treatment due to the fact that some level of pollutant removal $(k>0)$ was observed on the storms used for calibration. Simulation results for TSS are consistent with the fact that the treatment 
model underestimates effluent TSS concentrations (Figure 15(c)). For TSS, four observed concentrations were reported below the detection limit $(10 \mathrm{mg} / \mathrm{l})$. Although observed concentrations fell outside the uncertainty limits, the model did predict effluent concentration values less or equal to the analytical detection limit. For TPH-D and Zn, all observed concentrations but the first one were reported below the detection limit. The detection limit was approximately $0.40 \mathrm{mg} / \mathrm{l}$ for TPH-D and $0.01 \mathrm{mg} / \mathrm{l}$ for $\mathrm{Zn}$. The model did predict some effluent concentration values below the detection limit (Figure 16(b) and Figure 16(d)). 

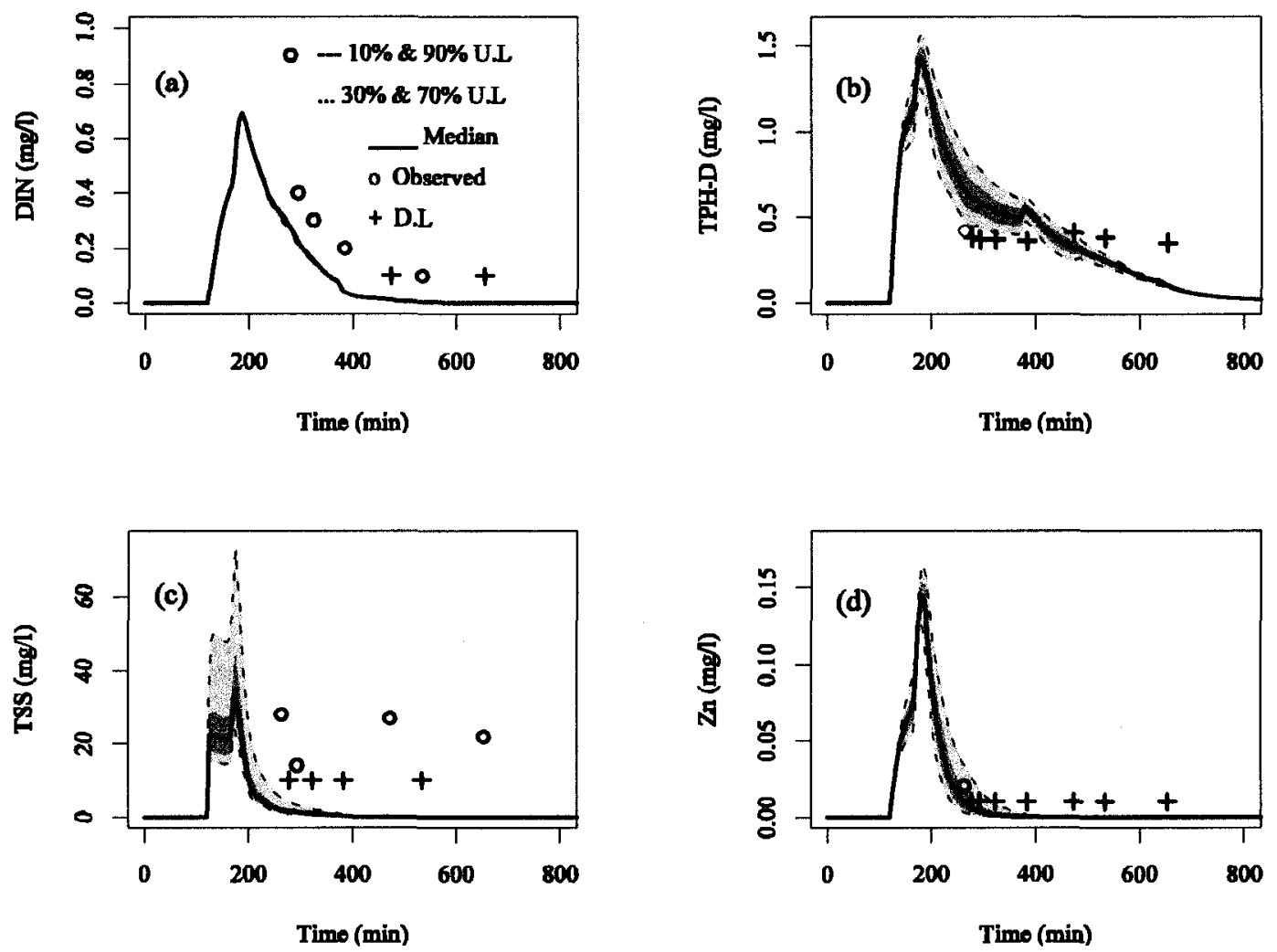

Figure 16 Monte Carlo simulations of predicted effluent pollutant concentrations for the 05/02/2006 storm event. U.L: Uncertainty Limits. D.L: Detection Limit. The dark shaded region indicates the $30 \%$ and $70 \%$ uncertainty limits. The light grey shaded region indicates the $10 \%$ and $90 \%$ uncertainty limits. Observed concentrations values and the reported detection limits were plotted as discrete points.

Figure 17 shows Monte Carlo simulation results for the 05/09/2006 storm event. As it happened for the 05/02/2006 event, the model did not accurately predict effluent DIN concentrations since they fell above the $90 \%$ uncertainty limit. However, the probable explanation for the poor model performance does not lie in the calibration procedure but in the conceptualization of the model approach for DIN. For this storm, all observed TPH-D effluent concentrations were reported below the detection limit. Effluent concentration values below the detection limit were predicted by the model (Figure 
17(b)). Few concentration values were reported above the detection limit for TSS and $\mathrm{Zn}$. The detection limit was approximately $10 \mathrm{mg} / \mathrm{l}$ for TSS and $0.01 \mathrm{mg} / \mathrm{l}$ for $\mathrm{Zn}$. The model was capable of predicting the first two samples for both TSS (Figure 17(c)) and Zn (Figure 17(d)) within the 90\% uncertainty limit. For these two contaminants, simulation results predicted concentrations values below the detection limit.
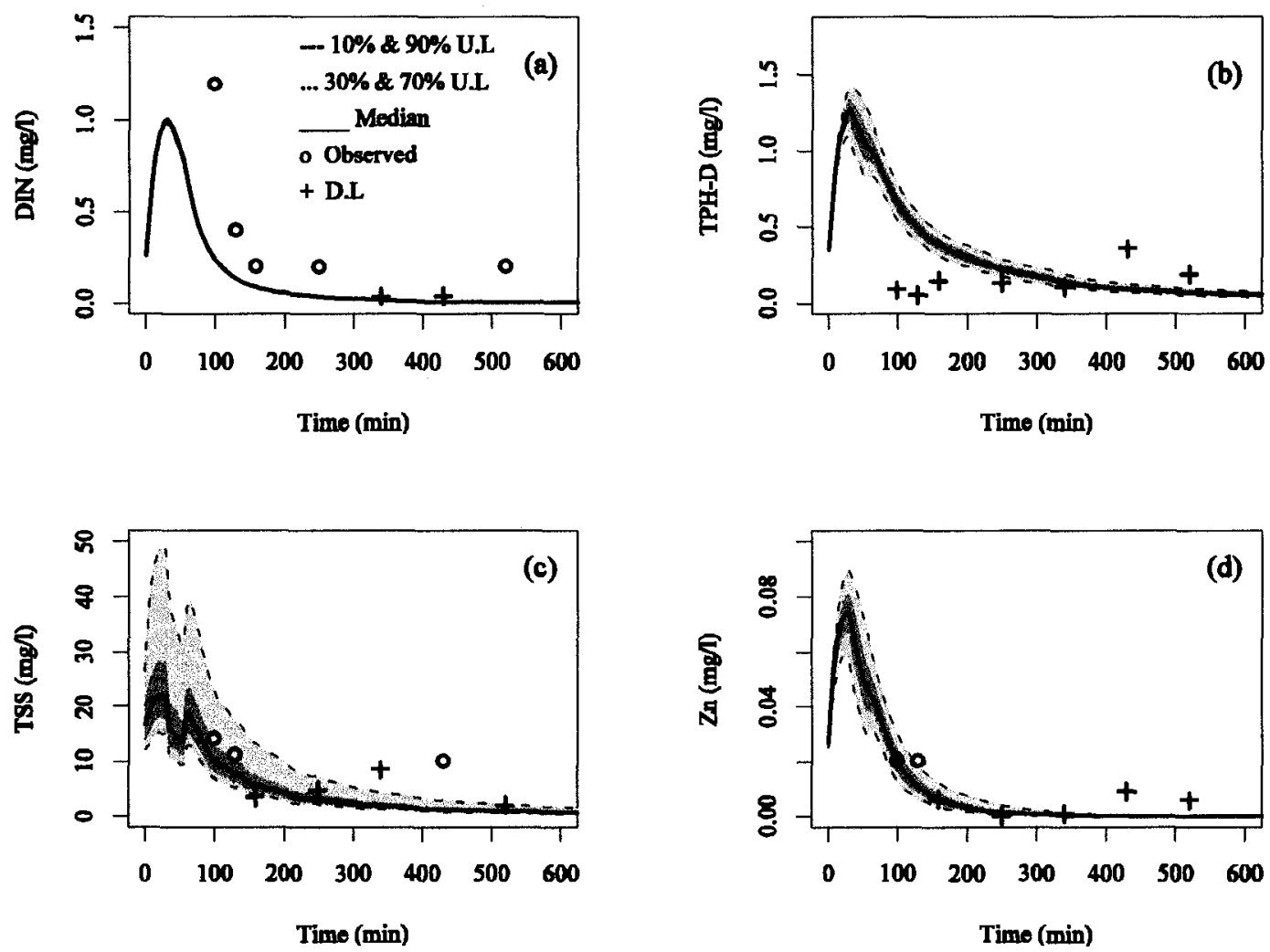

Figure 17 Monte Carlo simulations of predicted effluent pollutant concentrations for the 05/09/2006 storm event. U.L: Uncertainty Limits. D.L: Detection Limit. The dark shaded region indicates the $30 \%$ and $70 \%$ uncertainty limits. The light grey shaded region indicates the $10 \%$ and $90 \%$ uncertainty limits. Observed concentrations values and the reported detection limits were plotted as discrete points. 
Table 14 shows the total observed and estimated effluent mass for the 5/2/2006 and 5/9/2006 storms. The observed mass was computed using two different approaches when a detection limit was reported: (1) using a zero concentration and (2) generating a concentration from the probability distributions fitted to the effluent data (Table 10). The three estimated values correspond to the $30 \%\left(q_{30}\right), 50 \%\left(q_{50}\right)$, and $70 \%\left(q_{70}\right)$ uncertainty limits. The model underestimated the total effluent mass TSS and DIN. The total estimated mass was higher than the observed mass for TPH-D and $\mathrm{Zn}$.

Table 14 Observed and estimated total effluent mass for the storms used for validation.

\begin{tabular}{ccccccc}
\hline \multirow{2}{*}{ Storm } & \multirow{2}{*}{ Pollutant } & \multicolumn{5}{c}{ Total Mass (g) } \\
\cline { 3 - 7 } 5/2/2006 & TSS & 631 & 735 & 12 & 15 & 20 \\
& TPH-D & 0.63 & 8.77 & 12.40 & 13.72 & 15.21 \\
& Zn & 0.03 & 0.24 & 0.03 & 0.05 & 0.09 \\
& DIN & 7.35 & 8.64 & 2.52 & 2.58 & 2.65 \\
$5 / 9 / 2006$ & TSS & 101 & 147 & 81 & 100 & 129 \\
& TPH-D & 0 & 2.04 & 6.10 & 6.74 & 7.33 \\
& Zn & 0.09 & 0.19 & 0.11 & 0.14 & 0.18 \\
& DIN & 6.31 & 6.47 & 1.91 & 1.93 & 1.95 \\
\hline
\end{tabular}

(1) A zero concentration was used when a DL (detection limit) was reported.

(2) Concentrations were determined from a fitted gamma probabiliy distribution (Table 10) when a DL was reported.

\section{Conclusions}

A decay model for pollutant treatment, with parameter removal rate $(k)$ and decay order $(n)$, was used to estimate effluent pollutant concentrations of a sand filter treating stormwater. The model was based on a water balance, was dynamic, and variations in space were not considered. The model was implemented for a zero, first, and second 
order approximation $(n=0,1,2)$. Simulation results were described for two different storm events. The model was developed with the intention of emulating the decay function observed in chemical kinetics and sand filtration.

Calibration of the model was performed on a storm-by-storm basis using events monitored during the 2004-2006 period. Optimized parameter values were found for each storm and provided for TSS, TPH-D, DIN, and Zn (Table 11). Examples of model performance were provided for two storms. These results suggest that the treatment process of TPH-D and TSS follows a second order decay model. It seems that a first order decay model represents the treatment process of $\mathrm{Zn}$. For the storms selected for calibration, the decay model was able to preserve the overall distribution of the observed effluent EMCs for DIN, TPH-D, and Zn; however, it underestimated the distribution of the observed TSS effluent EMCs. The model could not adequately estimate effluent DIN concentrations of two storm events selected for validation.

Calibrated parameter values reported in this study can be used for modeling purposes. A Monte Carlo simulation technique was implemented to assess water quality performance of the treatment model. For this purpose, a gamma distribution was fitted to the optimized removal rate values (Figure 14). Afterward, values for the removal rate parameter (k) were drawn from the gamma distributions. For some examples, the model was capable of predicting concentrations above and below the analytical detection limit. Simulation results suggest that the two-parameter model may be sufficient to describe the overall mechanisms of treatment within the sand filter. However, the decay model should not be used beyond its limitations; in particular, if a detailed description of the physical and chemical processes is required.

Model results should be used carefully when comparing performance to other sand filters. It is has been reported that sediment particle size affects treatment performance 
as well as influent concentrations (Minton, 2002, EPA, 2002). Consequently, parameter values may be affected by those factors.

The statistical results provided in this study could be used in more complex models such as Bayesian applications. Additionally, one could add more complexity to the treatment model by incorporating other parameters that account for spatial variation and other transport and removal processes (for example the advection-dispersion transport equation). Further research should also explore the variables that affect the variation of the removal rate and the decay order. The treatment model can also be applied on other stormwater treatment systems such as: bioretention, retention ponds, gravel wetlands, and swales. 


\title{
CHAPTER 4
}

\section{MODELING URBAN STORMWATER QUALITY TREATMENT OF A GRAVEL WETLAND, A RETENTION POND, AND A SAND FILTER}

\begin{abstract}
Results of the application of a mathematical model for simulating contaminant removal from a group of stormwater treatment systems are presented. The stormwater treatment systems included: a gravel wetland, a retention pond, and a sand filter. The mathematical model was based on the mass balance principle and the assumption that an n-order decay model describes the complex processes of pollutant removal (for example sedimentation, biodegradation, filtration, plant uptake, and chemical precipitation). The model was defined by the parameters of removal rate $(k)$ and the decay order $(n)$. For each treatment system, a collection of storm events was monitored between 2004 and 2006. Monitoring of the treatment systems was performed in a side by side fashion so that each system received the same stormwater quantity and quality. This configuration made possible a comparison of the calibrated parameters obtained for each system. The runoff constituent analyses included: total suspended solids (TSS), total petroleum hydrocarbons - diesel range hydrocarbons (TPH-D), dissolved inorganic nitrogen (DIN, comprised of nitrate, nitrite, and ammonia), and zinc ( $\mathrm{Zn})$. The research objective was to determine the best set of parameters for each system in a storm-bystorm fashion and compare the distribution of the removal rate (k) among systems. The best set of parameters of the decay model was determined by using a simulated
\end{abstract}


annealing technique as part of the optimization process. Monte Carlo simulations were performed to describe the variability of the estimated effluent concentrations. It was found that first and second order decay models were more likely to describe the observed effluent concentrations. A significance difference between the distribution of the gravel wetland TSS removal rates and those calculated for the sand filter and the retention pond was obtained. This statistically difference among the distributions was also observed for DIN. No significance difference among the distribution of the removal rate (k) was observed for TPH-D. Only for the sand filter, the distribution of the Zinc removal rate $(k)$ was statistically different than those obtained for the gravel wetland and the retention pond.

\section{Introduction}

Stormwater runoff management aims to retain predevelopment hydrological and water quality characteristics. Watershed protection programs normally include some sort of stormwater management measure to help minimize impacts to natural systems resulting from land development. The effectiveness and performance of these management practices has been evaluated and documented for a variety of applications (Kayhanian et al., 2005; Roseen et al., 2006; Ice, 2004). In addition to the documentation on the performance of the stormwater systems, watershed managers need to be provided with different tools to evaluate system implementation. Mathematical models can help conceptualize the behavior of a particular environmental system and can be used as a powerful tool for predicting the system's response to a variety of conditions.

Arabi et al. (2006) calibrated and validated the Soil and Water Assessment Tool (SWAT) model to evaluate the effectiveness of various Best Management Practices 
(BMPs). Grassed waterways, grade stabilization structures, field borders, and parallel terraces were used to examine the transport of total suspended solids (TSS), total phosphorus (TP), and total nutrients (TN) on a selected group of watersheds. The mathematical model integrated the SCS curve number method and the Modified Universal Soil Loss Equation (MUSLE) to perform the analysis. Calibrated parameter values were obtained for each BMP and it was advised that the use of uncertainty analysis was key factor during the decision-making process.

Multivariate regression analysis has also been utilized for assessing water quality performance of stormwater measures. Edwards et al. (1996) fitted a non-linear regression model to estimate time variations of effluent pollutant concentrations from a collection of agricultural BMPs. The contaminants of concerned included: nitrate $\left(\mathrm{NO}_{3}\right.$ $\mathrm{N}$ ), ammonia $\left(\mathrm{NH}_{3}-\mathrm{N}\right)$, total kjeldahl nitrogen (TKN), total phosphorous (TP), total suspended solids (TSS), and chemical oxygen demand (COD). Decreasing trends were identified for $\mathrm{NH}_{3}-\mathrm{N}, \mathrm{TKN}$, and COD.

Some difficulties have been identified for the analysis of stormwater quality data and model application: sparse knowledge concerning the processes involved, lack of data, and difficulty in calibration (Ahyerre et al., 1998). Additionally, depending on the measuring method and the sampling procedures, some degree of uncertainty may be present. Difficulties in calibration occur when different vectors of model parameters seem to describe the recorded data. An uncertainty analysis of the predicted values is concerned with the variability of the target variable for a range of likely parameter values. For example, if the target variable is the effluent concentration, then an uncertainty analysis should describe the variability of the estimated concentrations predicted by the model.

Wang et al. (2006) investigated the application of a first order decay model to describe water quality of some stormwater treatment measures. In their model, (k) is the 
areal decay rate constant (m/year) and was used to describe the pollutant removal capabilities of the stormwater systems. Steady state flow conditions are assumed so that short-term flow and concentration fluctuations are negligible (Kadlec and Knight, 1996). Wang et al. (2006) did not report calibrated decay rates but recommended the model as a lumped approach to describing the overall water quality treatment processes within the systems. Further research was recommended to explore the variability of the model parameters under different real hydrodynamic conditions (for example influent loading and flow).

Influent flow and contaminant concentrations normally change over time as they enter the stormwater treatment system. Also, changes in the effluent concentration over time are expected as the treated water leaves the system. First order decay models have been used to estimate effluent pollutant concentrations from wet ponds (Wang et al., 2004). The combined effects of several pollutant removal mechanisms were represented by the removal rate parameter. This model was not intended to describe the physicochemical means by which sedimentation, filtration, or plant uptake occur within the treatment system. In stormwater, the effect of a decay order different than one is yet to be studied.

In this paper, the application of an n-order decay model to estimate effluent pollutant concentrations from stormwater systems was explored. The parameters of the model were the removal rate $(k)$ and the decay order $(n)$ (Avellaneda et al., 2008b). The contaminants of interest were: total suspended solids (TSS), total petroleum hydrocarbons - diesel range hydrocarbons (TPH-D), dissolved inorganic nitrogen (DIN, comprised of nitrate, nitrite, and ammonia), and zinc ( $\mathrm{Zn})$. The stormwater systems of the study included: a gravel wetland, a retention pond, and a sand filter. Each system received the same influent runoff from a commuter parking lot located in Durham, $\mathrm{NH}$. The data consisted of flow and water quality information of storms monitored between 
2004 and 2006. The objective was (i) to calibrate the treatment model and obtain parameter values on a storm-by-storm basis, (ii) to identify probability distribution functions for the reaction rate (k) and establish differences among the systems, and (iii) to study model uncertainty by performing Monte Carlo simulations and comparing the distribution of the observed and estimated effluent Event Mean Concentrations. The stormwater literature recommends the Event Mean Concentration (EMC) of a storm event as an index to characterize the total pollutant mass that enters or leaves a stormwater system (Urbonas, 1995; EPA, 2002).

\section{Stormwater Treatment Measures}

Three stormwater management measures were selected for this study: a retention pond, a sand filter, and a gravel wetland. The design parameters were taken from the New York State Stormwater Management Design Manual (2001). A pretreatment sedimentation basin was constructed for every system. The sedimentation basin helps remove large particles, provides for some flow equalization, and prevents the systems from premature clogging. The designed Water Quality Volume was $92.5 \mathrm{~m}^{3}$ (WQV), which represents $25 \mathrm{~mm}$ of precipitation over an impervious area of $4047 \mathrm{~m}^{2}$, and corresponds to the daily storm volume not exceeded $90 \%$ of the time on days with measurable precipitation.

\section{Gravel Wetland}

The gravel wetland is considered an infiltration/filtration system. The system is comprised of a sedimentation forebay and two horizontal-flow treatment cells. The filter 
media is a combination of a gravel substrate at the bottom $\left(0.6 \mathrm{~m}\right.$ thick and $D_{50}=19$ $\mathrm{mm}$ ) and a wetland soil on top. A $0.15 \mathrm{~m}$ perforated pipe is used to distribute the influent flow across the width of the subsurface gravel bed. Basic areal dimensions of the studied gravel wetland are $4.6 \mathrm{~m} \times 9.8 \mathrm{~m}$ for the filter basin footprint (for each cell) and $11.3 \mathrm{~m} \times 17.1 \mathrm{~m}$ for the forebay footprint. Stormwater flows horizontally through the gravel substrate and is collected by subdrains on the far end of the second treatment cell. The forebay was designed to hold $10 \%$ of the WQV while each treatment cell holds $45 \%$. The total area of the gravel wetland is $507 \mathrm{~m}^{2}$. Contaminants are removed by filtration, biological uptake, and adsorption.

\section{Retention Pond}

Retention ponds are designed to retain a large volume of stormwater and slowly release it. Treatment is achieved by physical settling of sediment and biological uptake. The areal dimensions of the studied retention pond are $14 \mathrm{~m} \times 21 \mathrm{~m}$. The treated runoff leaves the system through a $0.15 \mathrm{~m}$ perforated standpipe with reducing coupling to $0.025 \mathrm{~m}$. The surface area of the retention pond is covered by algae or aquatic plants. Side slopes were stabilized with grass, and spillways with stone and geotextile.

\section{Sand Filter}

The sand filter bed is $0.6 \mathrm{~m}$ thick, uses coarse to medium grain sand $\left(D_{50}=0.7 \mathrm{~mm}\right)$, and has areal dimensions of $2.4 \mathrm{~m} \times 6.1 \mathrm{~m}$. Temporary ponding of the filter basin is expected during larger storm events due to saturation of the filter media and the fact that inflow exceeds outflow. The sedimentation forebay was designed to hold $25 \%$ of the 
WQV while the filter basin holds the remaining $75 \%$.The filter bed is sub-drained by a $0.15 \mathrm{~m}$ perforated pipe bedded in a $0.20 \mathrm{~m}$ layer of crushed stone $\left(D_{50}=19 \mathrm{~mm}\right)$.

\section{Monitoring}

These three stormwater treatment measures were monitored between August 2004 and September 2006. A total of 15 storms were collected for the gravel wetland, 15 storms for the retention pond, and 16 storms for the sand filter. Automated samplers (6712SR ISCO) performed the sampling. Up to 24 samples were taken for each system, for each storm; however, normally 8-12 samples were used to characterize both influent and effluent stormwater quality for each system. The sampling program for each system was based on analyses of various effluent hydrographs. Influent and effluent flows were measured with inline weirs.

Runoff constituent analysis included: total suspended solids (TSS), total petroleum hydrocarbons - diesel range hydrocarbons (TPH-D), dissolved inorganic nitrogen (DIN, comprised of nitrate, nitrite, and ammonia), and zinc ( $\mathrm{Zn})$. Stormwater samples were analyzed by a laboratory that is state-certified for drinking water and wastewater. Guidance documents on collecting environmental data and the site quality assurance project plan were followed to assure good quality of the results. Table 15 shows characteristics of the storm events selected for this study. Note that the storm characteristics were obtained for the total watershed area; however, each system treated an equivalent fraction of the total storm volume. More description about the monitoring program is presented in Roseen et al. (2006). 
Table 15 Summary of monitored storm events

\begin{tabular}{|c|c|c|c|c|c|c|c|}
\hline $\begin{array}{l}\text { Rainfall } \\
\text { Event } \\
(\mathrm{m} / \mathrm{d} / \mathrm{y})\end{array}$ & $\begin{array}{c}\text { Peak } \\
\text { Intensity } \\
\text { (mm/hr) }\end{array}$ & $\begin{array}{c}\text { Rain } \\
\text { Duration } \\
\text { (min) }\end{array}$ & $\begin{array}{l}\text { Total } \\
\text { Depth } \\
(\mathrm{mm})\end{array}$ & $\begin{array}{c}\text { Peak } \\
\text { Flow } \\
\text { (m²/day) }\end{array}$ & $\begin{array}{c}\text { Volume } \\
\left(\mathrm{m}^{3}\right)\end{array}$ & $\begin{array}{c}\text { Anticedent } \\
\text { Dry Period } \\
\text { (days) }\end{array}$ & Season \\
\hline $9 / 18 / 2004$ & 15 & 1075 & 50 & 5642 & 1364 & 7.0 & Fall \\
\hline $10 / 30 / 2004$ & 21 & 705 & 11 & 8678 & 281 & 13.0 & Fall \\
\hline $11 / 24 / 2004$ & 9 & 705 & 18 & 4394 & 530 & 3.5 & Fall \\
\hline $1 / 14 / 2005$ & 24 & 645 & 17 & 21101 & 1033 & 1.3 & Winter \\
\hline $2 / 10 / 2005$ & 6 & 1520 & 32 & 4437 & 795 & 3.6 & Winter \\
\hline $3 / 8 / 2005$ & 3 & 1220 & 20 & 2338 & 406 & 5.7 & Winter \\
\hline $3 / 28 / 2005$ & 12 & 1685 & 60 & 7675 & 3082 & 3.4 & Winter \\
\hline $4 / 20 / 2005$ & 12 & 480 & 15 & 4274 & 1017 & 5.9 & Spring \\
\hline $6 / 22 / 2005$ & 15 & 95 & 8 & 9120 & 266 & 4.0 & Summer \\
\hline $8 / 13 / 2005$ & 24 & 765 & 13 & 18408 & 514 & 10.0 & Summer \\
\hline $9 / 15 / 2005$ & 18 & 30 & 5 & 5518 & 86 & 10.0 & Fall \\
\hline $11 / 6 / 2005$ & 12 & 100 & 7 & 2801 & 135 & 10.8 & Fall \\
\hline $11 / 30 / 2005$ & 9 & 810 & 18 & 2503 & 363 & 5.0 & Fall \\
\hline $12 / 16 / 2005$ & 18 & 630 & 35 & 2561 & 458 & 5.5 & Winter \\
\hline $1 / 11 / 2006$ & 15 & 320 & 15 & 2903 & 214 & 5.8 & Winter \\
\hline $2 / 17 / 2006$ & 12 & 110 & 3 & 1493 & 37 & 2.5 & Winter \\
\hline $3 / 13 / 2006$ & 12 & 170 & 7 & 1345 & 75 & 2.5 & Winter \\
\hline $5 / 2 / 2006$ & 12 & 1920 & 60 & 4642 & 1331 & 7.0 & Spring \\
\hline $5 / 9 / 2006$ & 3 & 565 & 14 & 1621 & 322 & 5.6 & Spring \\
\hline $6 / 1 / 2006^{*}$ & 125 & 485 & 51 & 27194 & 930 & 10.7 & Summer \\
\hline $6 / 21 / 2006$ & 27 & 80 & 5 & 5190 & 93 & 4.7 & Summer \\
\hline $7 / 22 / 2006$ & 40 & 50 & 5 & 8333 & 81 & 7.5 & Summer \\
\hline $9 / 6 / 2006$ & 30 & 585 & 16 & 6087 & 410 & 4.5 & Fall \\
\hline
\end{tabular}




\section{Methodology}

\section{Stormwater Treatment Model}

An n-order decay model was implemented to estimate effluent pollutant concentrations from the stormwater treatment systems. The parameters of the model were the removal rate (k) and the decay order $(n)$. The treatment system was conceptualized as a black box where influent and effluent variables must be determined or measured (Avellaneda et al., 2008b). The partial differential equation that describes time variations of effluent pollutant concentrations can be written as follows:

$$
\frac{d C_{\text {out }}}{d t}=\frac{C_{\text {in }} Q_{\text {in }}-C_{\text {out }} Q_{\text {out }}}{S}-\frac{C_{\text {out }}}{S} \frac{d S}{d t}-k C_{\text {out }}^{n}
$$

where $C_{\text {out }}$ represents the effluent concentration $(\mathrm{mg} / \mathrm{l}) ; \mathrm{C}_{\text {in }}$ is the influent concentration $(\mathrm{mg} / \mathrm{l}) ; Q_{\text {in }}$ is the influent flow rate $\left(\mathrm{m}^{3} /\right.$ day); $Q_{\text {out }}$ is the effluent flow rate $\left(\mathrm{m}^{3} /\right.$ day); $S$ represents the storage within the system $\left(\mathrm{m}^{3}\right) ; \mathrm{dS} / \mathrm{dt}$ is the change in storage $\left(\mathrm{m}^{3} / \mathrm{day}\right) ; \mathrm{t}$ is time (days); $\mathrm{k}$ is the removal rate $\left(\left(\mathrm{mg} / \mathrm{l}^{-\mathrm{n}+1} / \mathrm{day}\right)\right.$; and $\mathrm{n}$ represents the decay order $(-)$. These units will be used throughout the paper.

The model was dynamic and spatial variations were not considered. Influent concentrations were computed by using an accumulation and washoff model (Avellaneda et al., 2008a). Ground water effects and other infiltration sources were not included since the systems were constructed in a low permeability, clay soil. 


\section{Parameter Estimation}

Model calibration requires an optimization technique in order to determine the group of parameter values that best estimate the measured effluent concentrations. The sumof-squares estimator was adopted as the objective function. This estimator is computed by summing up the squares of the residuals between observed and estimated concentrations:

$$
O(\Theta)=\min \sum_{i=1}^{m}\left(C_{o u t, o b s}^{i}-C_{o u t, e s t}^{i}(\Theta)\right)^{2}
$$

where $O$ denotes the objective function, $\Theta$ represents the vector of parameter values $(k$ and $n$ ); $C_{\text {out,obs }}$ are the measured effluent concentrations; $C_{\text {out,est }}$ are the estimated effluent concentrations obtained from the stormwater treatment model; i denotes the current sample; and $\mathrm{m}$ is the number of samples taken during the storm event. The optimization algorithm extensively searches the parameter space until a minimum is found. In this study, the simulated annealing (SA) algorithm was utilized as the optimization technique (Kirkpatrick et al., 1983). The SA algorithm is a stochastic method that locates the parameter values that minimize or maximize a given objective function. Avellaneda et al. (2008b) described the algorithm with application to a stormwater treatment model.

\section{Measured Concentrations Below Detection Limit}

A detection limit is reported when pollutant concentrations are below the analytical reporting limit of the laboratory. However, a range of concentrations may not be practical 
for modeling purposes. Table 16 summarizes, for each system and contaminant, the number of samples reported as below detection limit (BDL). Normally, the following detection limits were reported for the different contaminants: $0.03 \mathrm{mg} / \mathrm{l}$ for $\mathrm{Zn}, 10 \mathrm{mg} / \mathrm{l}$ for TSS, $0.3 \mathrm{mg} / \mathrm{l}$ for TPH-D, and $0.05 \mathrm{mg} / \mathrm{l}$ for DIN. The detection limit changed depending on the real sample volume available.

Table 16 Summary of effluent samples with concentrations reported as BDL.

\begin{tabular}{cccccc}
\hline \multirow{2}{*}{ System } & \multirow{2}{*}{$\begin{array}{c}\text { Total Number } \\
\text { of Samples }\end{array}$} & \multicolumn{4}{c}{ BDL Samples } \\
\cline { 3 - 6 } & & TPH-D & Zn & DIN & TSS \\
\hline Gravel Wetland & 136 & 131 & 116 & 95 & 126 \\
Retention Pond & 117 & 71 & 71 & 46 & 35 \\
Sand Filter & 132 & 97 & 61 & 27 & 37 \\
\hline
\end{tabular}

One way of overcoming this issue is by generating concentrations for BDL data points from a probability distribution function (PDF) that describes the statistical characteristics of the concentration values reported above the detection limit. This approach aims at preserving the statistical properties of the sample population. Figures 18, 19, 20 show the empirical (solid line) cumulative distribution functions (CDFs) for the different treatment systems and contaminants considered in this study. A gamma probability distribution was fitted to the data (dashed line). Additionally, a $95 \%$ confidence interval was computed and the Kolmogorov-Smirnov test (K-S test) performed to evaluate the goodness-of-fit of the distribution (Kottegoda and Rosso, 1997). The fitted CDF was accepted if $D \leq D_{\text {critical. }}$ Critical values were obtained using the following approximation: $D_{\text {critical }}=1.36 / \mathrm{m}^{0.5}$ for $\mathrm{m}>40$ (m number or samples); and from tabulated values in any other case (Miller, 1956). The parameters of the fitted distributions are shown in Table 17. Note that a high percentage of the effluent concentrations collected for the gravel 
wetland were reported below the detection limit. The $D$ statistic was slightly higher than the $D_{\text {critical }}$ on the fitted probabilities distributions for gravel wetland $(\mathrm{Zn})$ and sand filter $(\mathrm{Zn})$. In general, the gamma distribution was deemed sufficiently accurate and versatile to represent each variable and each system.
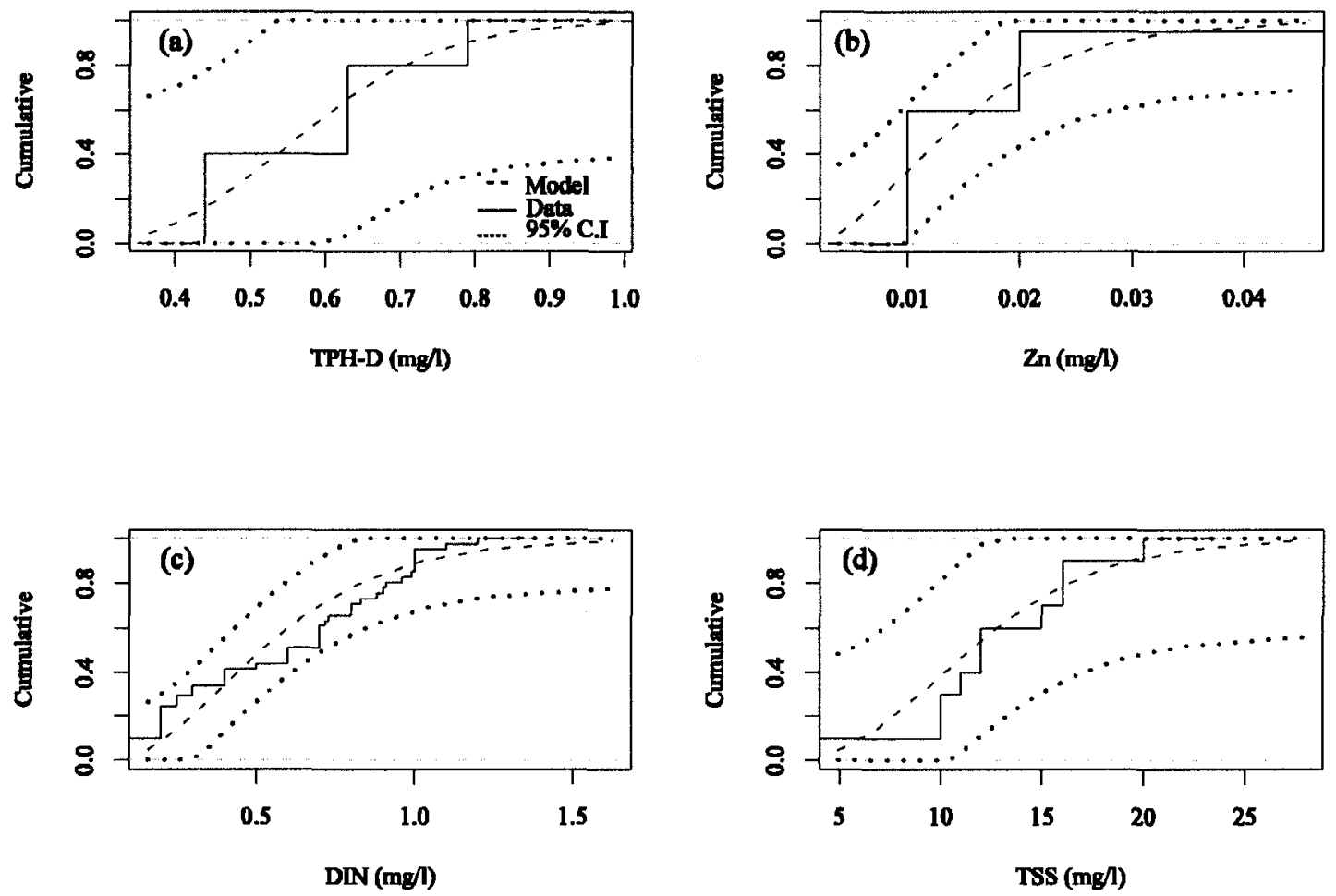

Figure 18 Empirical and theoretical CDF of effluent concentrations for the gravel wetland. C.I: Confidence Interval. 

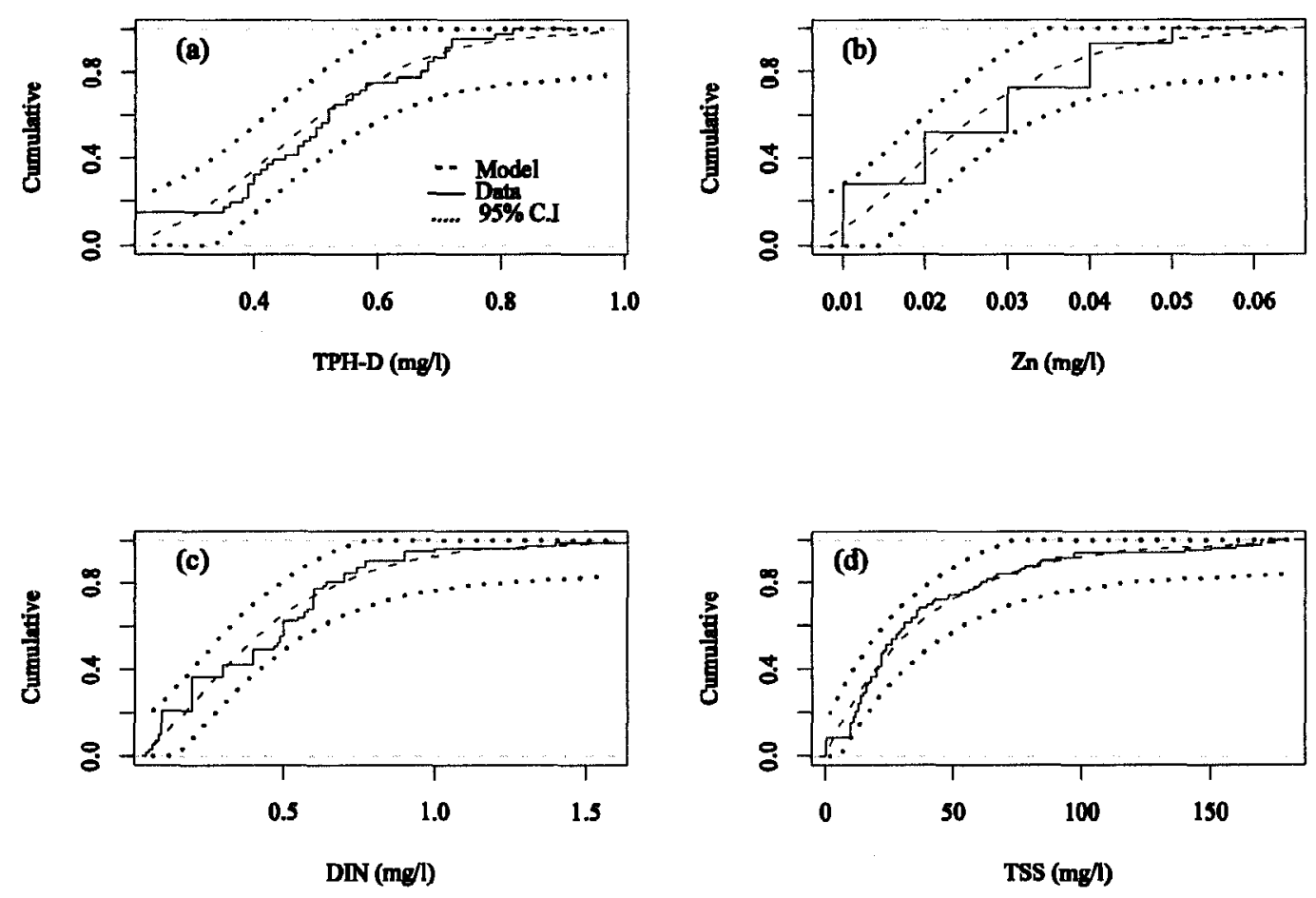

Figure 19 Empirical and theoretical CDF of effluent concentrations for the retention pond. C.I: Confidence Interval.
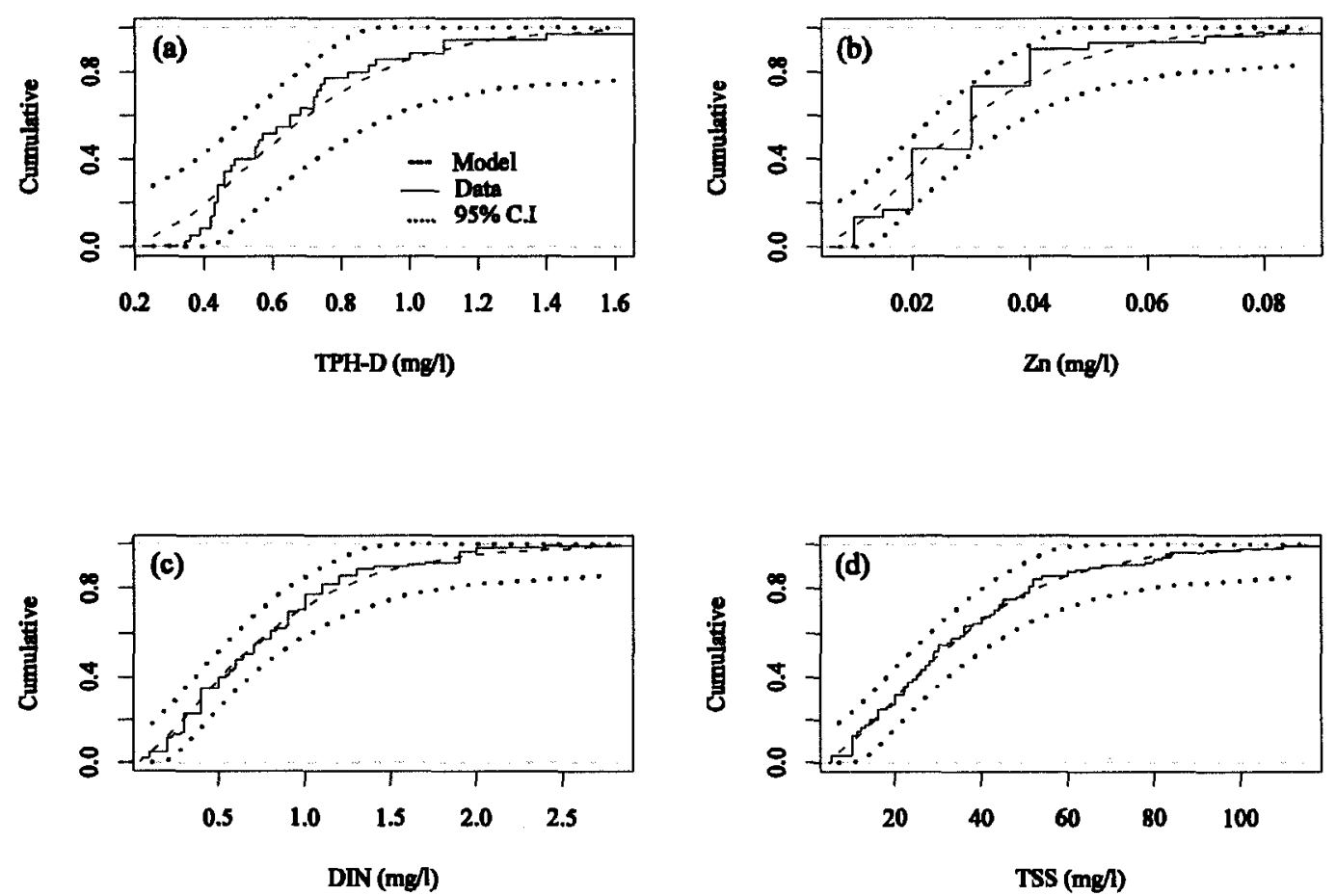

Figure 20 Empirical and theoretical CDF of effluent concentrations for the sand filter. C.I: Confidence Interval. 
Table 17 Parameters of the gamma distributions fitted to the observed concentrations.

\begin{tabular}{ccccccc}
\hline System & Contaminant & $\begin{array}{c}\text { Total samples } \\
\text { above DL (i) }\end{array}$ & $\gamma$ (shape) & $\theta$ (scale) & $D_{\text {critical }}$ & $D_{\mathrm{i}, \alpha}$ \\
\hline Gravel Wetland & TPH-D & 5 & 15.59 & 0.04 & - & 0.25 \\
& Zn & 20 & 2.69 & 0.01 & 0.29 & 0.32 \\
& DIN & 41 & 3.00 & 0.19 & 0.21 & 0.19 \\
& TSS & 10 & 5.22 & 2.34 & 0.41 & 0.29 \\
Retention Pond & TPH-D & 46 & 7.75 & 0.06 & 0.20 & 0.13 \\
& Zn & 46 & 3.89 & 0.01 & 0.20 & 0.20 \\
& DIN & 71 & 1.80 & 0.25 & 0.16 & 0.13 \\
& TSS & 82 & 0.99 & 39.26 & 0.15 & 0.14 \\
Sand Filter & TPH-D & 35 & 4.61 & 0.15 & 0.23 & 0.13 \\
& Zn & 71 & 2.76 & 0.01 & 0.16 & 0.17 \\
& DIN & 105 & 1.72 & 0.46 & 0.13 & 0.07 \\
& TSS & 95 & 2.12 & 16.67 & 0.14 & 0.07 \\
\hline
\end{tabular}

Level of significance $\alpha=0.05$

"-" indicates that value is not available due to the sample size

\section{Results}

\section{Model Application}

A group of storm events was selected for model calibration on each treatment system. Note that water quality data for all the contaminants were not available for all the storms; additionally, not exactly the same storm events were considered for all the systems due to maintenance or site issues. For calibration purposes: 14 storm events were selected for the gravel wetland; 14 events for the retention pond; and 15 for the sand filter. Moreover, one storm event was left for model validation for each system.

The stormwater treatment model, Equation (13), was calibrated on a storm-by-storm basis with the hypothesis that different parameter values might be obtained for a variety 
of storm intensities, pollutant loading, antecedent dry period, volume, etc. Figure 21 displays a statistical comparison of the removal rate ( $\mathrm{k}$ has units of $\left(\mathrm{mg} / \mathrm{l}^{-\mathrm{n}+1} /\right.$ day) for the gravel wetland $(G W)$, retention pond $(R P)$, and sand filter (SF). Lower DIN removal rates were obtained for the sand filter probably due to the lack of vegetation. Median DIN removal rates ranged as follows: $0.3-0.7(\mathrm{SF}), 0.006-0.1(\mathrm{RP})$, and $4-10(\mathrm{GW})$. The gravel wetland shows a slightly higher median TPH-D removal rate. Median TPH-D removal rates ranged as follows: $14-30$ (SF), $0.008-71(\mathrm{RP})$, and $0.5-46(\mathrm{GW})$. For TSS, the highest median removal rate was obtained for the gravel wetland. The median TSS removal rates ranged as follows: $0.003-52$ (SF), $0.003-21$ (RP), and $4-45$ (GW). The highest median $\mathrm{Zn}$ removal rates were achieved by the sand filter and gravel wetland. Median $\mathrm{Zn}$ removal rates ranged as follows: $30-75$ (SF), $20-23$ (RP), and 0.02 - 37 (GW). Outliers were identified and are displayed in Figure 21. 


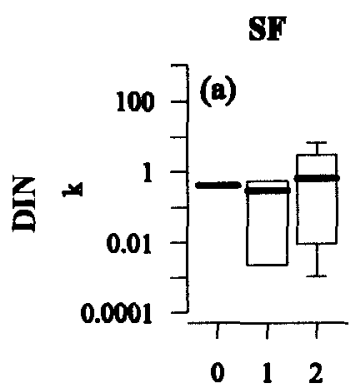

$\mathbf{n}$

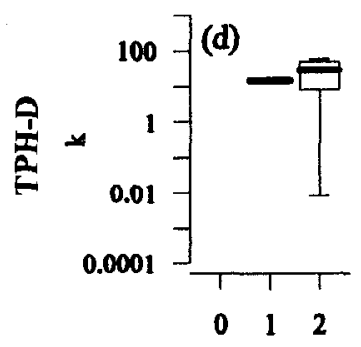

$\mathbf{n}$

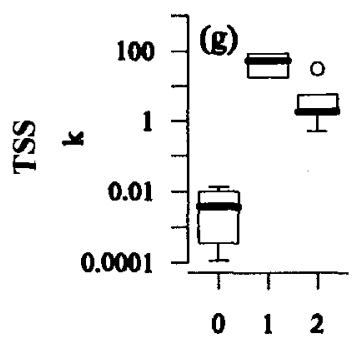

n

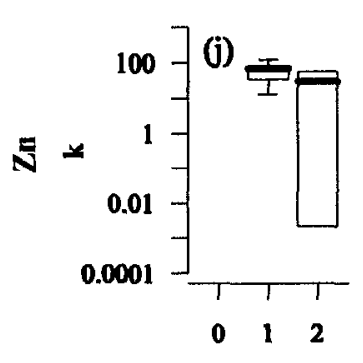

n

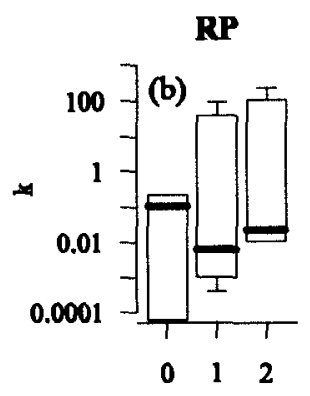

n

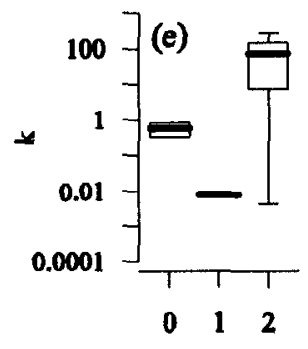

n

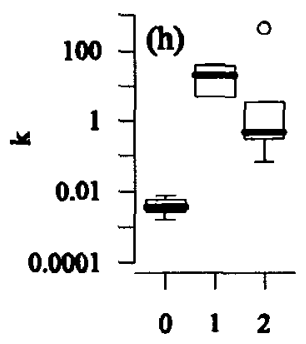

n

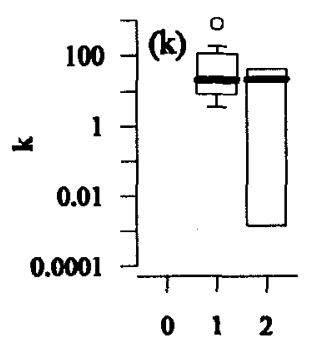

n

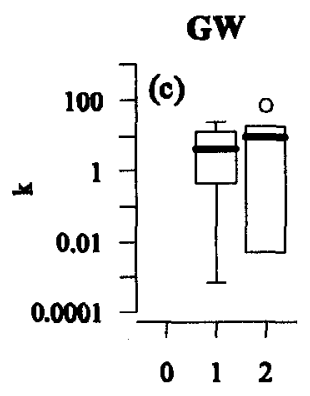

n

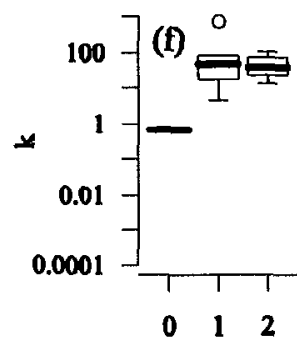

- Outlier Maximum

$75 \%$

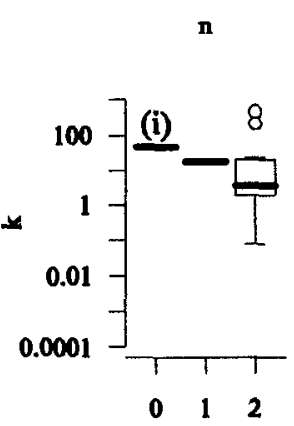

n

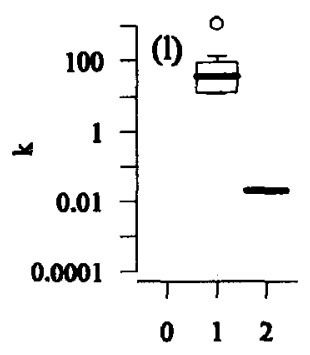

n

Figure 21 Box and whisker plot of the removal rate (k) optimized parameter values for all the contaminants and decay order models $(n=0,1,2)$. SF: Sand Filter; RP: Retention Pond; and GW: Gravel Wetland. The removal rate has units of $\left(\left(\mathrm{mg} / \mathrm{l}^{-\mathrm{n}+1} / \mathrm{day}\right)\right.$. 


\section{Statistical Analysis}

A gamma probability distribution with parameters $\gamma$ (shape) and $\theta$ (scale) was fitted to the optimized removal rates. For this purpose, the empirical cumulative distribution function (CDF) was found and the parameters of the gamma distribution computed. The K-S test was then performed to compare the fitted gamma distribution (Model) to the empirical CDF (Data). Table 18 shows statistical results for all the systems and contaminants. The fitted distributions for Zn (Gravel wetland) and DIN (Retention pond) did not pass the K-S test for a level of significance $\alpha=0.05$ ( $D \geq D_{\text {critical }}$ ). Figure 22 shows the CDF obtained for the gravel wetland, retention pond, and sand filter. The empirical CDF (Data) and the fitted gamma distribution (Model) are plotted along with a $95 \%$ confidence interval. Table 19 shows the frequency of optimized decay orders for the different contaminants and systems.

Table 18 Parameters of the gamma distributions fitted to optimized removal rate values. Critical values were obtained from Miller (1956).

\begin{tabular}{ccccccc}
\hline System & Contaminant & $\begin{array}{c}\text { Total Number } \\
\text { of Storms (i) }\end{array}$ & $\gamma$ (shape) & $\theta$ (scale) & $D_{\text {critical }}$ & $D_{i, \alpha}$ \\
\hline Gravel Wetland & TPH-D & 13 & 0.2 & 393 & 0.36 & 0.34 \\
& Zn & 14 & 0.2 & 740 & 0.35 & 0.47 \\
& DIN & 13 & 0.4 & 31 & 0.36 & 0.31 \\
& TSS & 14 & 0.2 & 285 & 0.35 & 0.24 \\
Retention Pond & TPH-D & 13 & 0.6 & 117 & 0.36 & 0.33 \\
& Zn & 14 & 0.2 & 439 & 0.35 & 0.32 \\
& DIN & 14 & 0.2 & 149 & 0.35 & 0.64 \\
Sand Filter & TSS & 13 & 0.1 & 354 & 0.36 & 0.23 \\
& TPH-D & 11 & 1.9 & 15 & 0.39 & 0.18 \\
& Zn & 13 & 2.4 & 25 & 0.36 & 0.21 \\
& DIN & 13 & 0.3 & 4 & 0.36 & 0.33 \\
& TSS & 12 & 0.2 & 52 & 0.37 & 0.18 \\
\hline
\end{tabular}

Level of significance $\alpha=0.05$ 

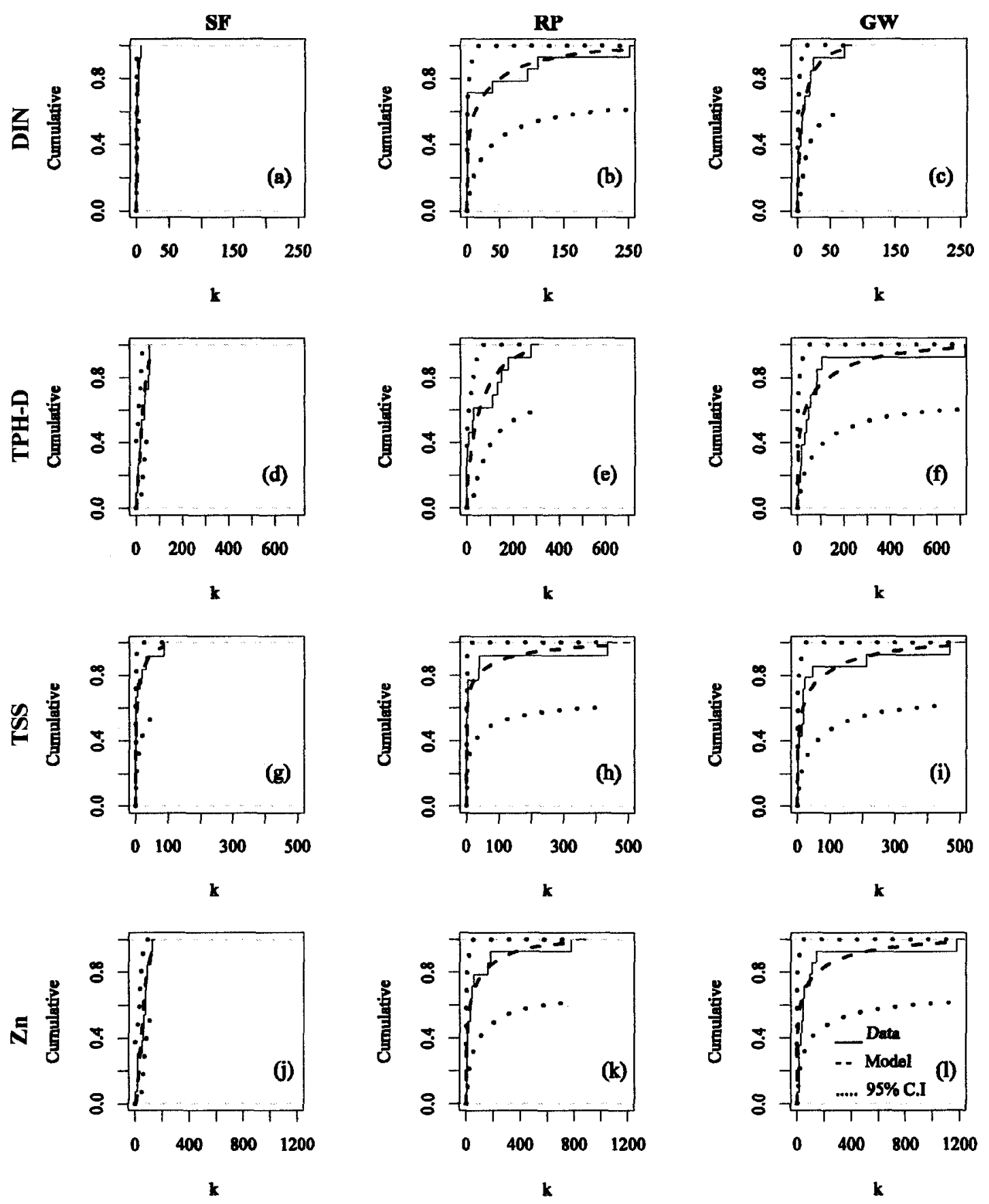

Figure $22 \mathrm{CDFs}$ of optimized removal rates ( $\mathrm{k}$ - Data) and fitted gamma distributions (Model) for the sand filter (SF), the retention pond (RP), and the gravel wetland (GW). The removal rate has units of $\left((\mathrm{mg} /)^{-n+1} / \mathrm{day}\right)$. C.I: Confidence Interval. 
Table 19 Frequency of optimized decay orders for the different contaminants and systems.

\begin{tabular}{|c|c|c|c|c|}
\hline \multirow{2}{*}{ System } & \multirow{2}{*}{ Contaminant } & \multicolumn{3}{|c|}{ Decay Order $(n)$} \\
\hline & & $\mathrm{n}=0$ & $n=1$ & $n=2$ \\
\hline \multirow[t]{4}{*}{ Sand Filter } & DIN & 3 & 2 & 8 \\
\hline & TPH-D & 0 & 1 & 10 \\
\hline & TSS & 4 & 2 & 6 \\
\hline & $\mathrm{Zn}$ & 0 & 11 & 2 \\
\hline \multirow[t]{4}{*}{ Retention Pond } & DIN & 2 & 6 & 6 \\
\hline & TPH-D & 2 & 1 & 10 \\
\hline & TSS & 3 & 4 & 6 \\
\hline & $\mathrm{Zn}$ & 0 & 12 & 2 \\
\hline \multirow[t]{4}{*}{ Gravel Wetland } & DIN & 0 & 7 & 5 \\
\hline & TPH-D & 1 & 5 & 7 \\
\hline & TSS & 1 & 2 & 11 \\
\hline & $\mathrm{Zn}$ & 0 & 13 & 1 \\
\hline
\end{tabular}

The fitted gamma CDFs to the removal rate were compared for each contaminant. For this purpose, the K-S test was conducted to assess whether or not for two systems the distributions were statistically different (for a level of significance $\alpha=0.05$ ). The $\mathrm{K}-\mathrm{S}$ test was selected due to the fact that no assumption about the distribution of the data was required (non-parametric test). Table 20 shows results from the statistical analysis. For DIN, no statistical difference was observed between the distribution of $k$ for the sand filter and the retention pond. The distribution for the gravel wetland was significantly different (higher $k$ values) than the distribution for the sand filter and the retention pond. The distribution of the TPH-D removal rate among the three systems was statistically indistinguishable. There was no statistical difference between the distribution of the TSS removal rate for the sand filter and the retention pond. However, the distribution for the 
gravel wetland was significantly different than that of the retention pond and sand filter. For $\mathrm{Zn}$, there was no statistical difference between the distribution of $\mathrm{k}$ for the gravel wetland and the retention pond. The distribution for the sand filter was significantly different than the distribution for the other two systems.

Table 20 Comparison of the fitted removal rate cumulative distribution function between two systems.

\begin{tabular}{cccccc}
\hline \multirow{2}{*}{$\begin{array}{c}\text { Contaminant } \\
\end{array}$} & $\begin{array}{c}\text { System } \\
\text { Combination }\end{array}$ & $\begin{array}{c}\text { Total number } \\
\text { of values (i) }\end{array}$ & $\mathrm{D}_{\text {critical }}$ & $\mathrm{D}_{\mathrm{i}, \alpha}$ & $\begin{array}{c}\text { Are the distributions } \\
\text { different? }\end{array}$ \\
\hline \multirow{2}{*}{ TPHD } & GW and RP & 13 & 0.36 & 0.31 & No \\
& GW and SF & 13 & 0.36 & 0.31 & No \\
& RP and SF & 11 & 0.39 & 0.38 & No \\
Zn & GW and RP & 14 & 0.36 & 0.36 & No \\
& GW and SF & 13 & 0.36 & 0.41 & Yes \\
& RP and SF & 13 & 0.36 & 0.41 & Yes \\
DIN & GW and RP & 13 & 0.36 & 0.41 & Yes \\
& GW and SF & 13 & 0.36 & 0.54 & Yes \\
& RP and SF & 13 & 0.36 & 0.29 & No \\
\multirow{3}{*}{ TSS } & GW and RP & 13 & 0.36 & 0.47 & Yes \\
& GW and SF & 12 & 0.37 & 0.44 & Yes \\
& RP and SF & 12 & 0.37 & 0.17 & No \\
\hline
\end{tabular}

Level of significance $\alpha=0.05$. Sand Filter (SF), Gravel Wetland (GW), and Retention Pond (RP).

A similar analysis was performed for the decay order parameter. In this case, the goal was to assess whether or not there was a difference between a first and a second-order decay model among the gravel wetland, the retention pond, and the sand filter. The null hypothesis was that there was no difference between the two models among the three systems. A zero-order decay approximation was not considered in the analysis since only for a few cases of this model seemed to be more appropriate. To perform the 
analysis, a contingency table was created and the $\chi^{2}$-test used for testing the difference between proportions of the two decay models. The contingency was created using the frequency counts for a first and a second order decay model displayed in Table 19.

For each contaminant, the $\chi^{2}$ value was estimated and compared against the critical value using (3-1) $\times(2-1)=2$ degrees of freedom (three systems and two models). The computed $\chi^{2}$ values were: 3.51 (DIN), 5.04 (TPH-D), 1.79 (TSS), and $0.51(\mathrm{Zn})$. The null hypothesis could not be rejected since those values were less than the critical value of the $\chi^{2}$ distribution for a level of significance $\alpha=0.05$ and two degrees of freedom $\left(\chi^{2}=6\right)$. Essentially, there was no evidence of a relationship between the two models and the systems. For example, a first-order decay model was found most frequently to be optimal for each of the systems. The $\chi^{2}$ value for TPH-D was close to its critical value since for the gravel wetland the frequency of a first and a second-order model was similar. Note that this test is most accurate when the expected frequency in each cell of the contingency table is at least five. However, as it is reported in Table 19, the observed frequency was less than five in some cells. For this reason, poor performance of the test might be expected.

Even though a rigorous statistical conclusion about the difference between the two decay models could not be achieved, a few descriptive statements can be drawn. The results indicated that the decay order $n$ varies among contaminants. Only for a few storms and contaminants, a zero-order reaction model seemed to best describe effluent concentrations. For TSS, the objective function was frequently minimized when a second-order decay model was used. For the gravel wetland, this characteristic was particularly appreciable since only for three storms the optimized decay order was other than two. A second-order decay model was also the best approximation for the retention 
pond and the sand filter; however, not as often as for the gravel wetland. The results for TPH-D suggested that a second-order decay model was likely to describe effluent concentrations. For the sand filter, a first-order decay approximation was found to be the optimal model only for one storm. For the retention pond, the optimum decay order was other than a second one only for three storm events. Moreover, for the gravel wetland, the frequency of a second-order decay model was slightly higher than the frequency obtained by a first-order model. A first-order decay model seemed to best represent effluent concentrations for $\mathrm{Zn}$. For all the stormwater systems, a second-order decay model was found to be the optimal only for a few storms. For the gravel wetland and the retention pond, the frequency of a first-order and a second-order decay model was similar for DIN. A second order decay model was more frequent for the sand filter.

\section{Event Mean Concentration (EMC)}

For this study, the distribution of the observed effluent EMCs was compared against the distribution of the estimated effluent EMCs. The EMC of a storm event is defined as the total pollutant load divided by the total runoff volume (Charbeneau and Barret, 1998). Observed effluent EMCs were computed for the systems using discrete samples collected throughout the duration of the storm event. Estimated effluent EMCs were computed using the estimated effluent pollutographs obtained from the decay treatment model (using optimized values of $k$ and $n$ ). The CDF for both the observed EMCs and the estimated EMCs was computed to assess the results obtained during calibration.

Figures 23, 24, and 25 show the CDFs (observed and estimated) for the gravel wetland, the retention pond, and the sand filter, respectively. Calibration results can be examined by comparing the estimated effluent CDF and the observed effluent CDF. It should be noticed that this comparison looks only at the calibration results and does not 
intend to validate the performance of the model, which should be assessed by analyzing storms not included during calibration.

CDFs for the gravel wetland are shown in Figure 23. Note that a large portion of the effluent samples were reported with concentrations below the detection limit (Table 16). For example, 131 samples out of 136 were reported with concentrations values below detection limit for TPH-D. The CDF was computed using the detection limit for BDL data.

DIN

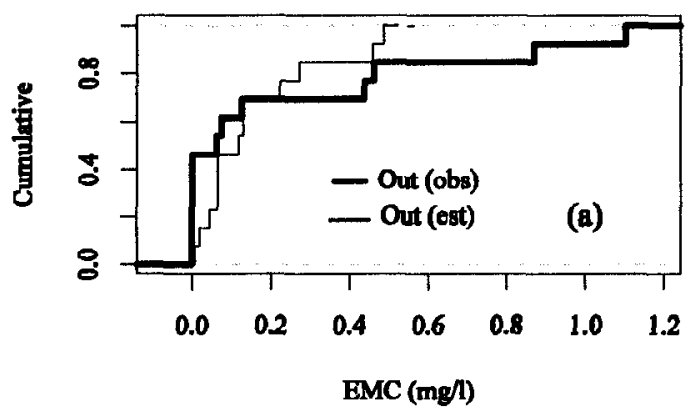

TSS

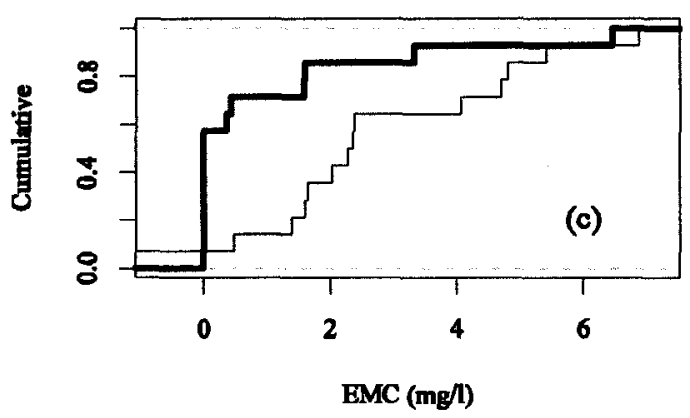

TPH-D

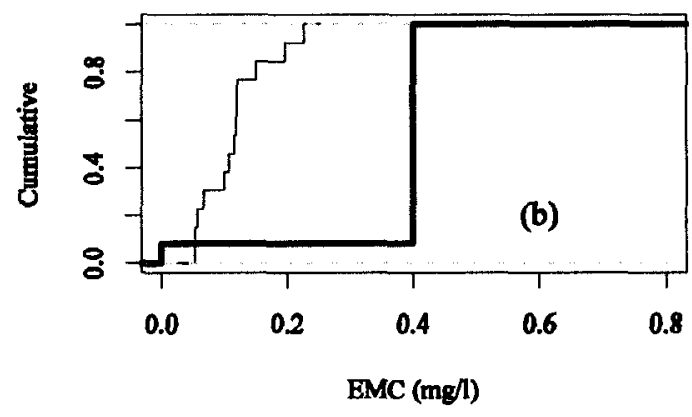

$\mathbf{Z n}$

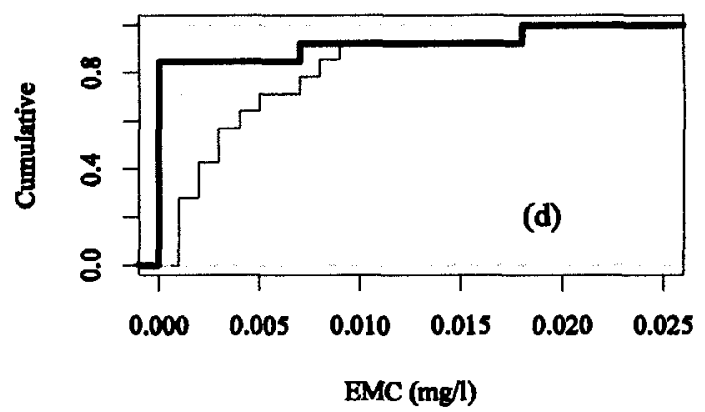

Figure 23 CDFs of Event Mean Concentrations (EMC) for the gravel wetland. Out: effluent; obs: observed; and est: estimated.

Results for the retention pond are displayed in Figure 24. The K-S test was performed to assess whether or not the estimated effluent CDF followed the distribution of the observed effluent CDF. The estimated effluent CDF for TPH-D did not pass the K-S test 
since $D_{n, \alpha}>D_{\text {critical }}$ for a level of significance $\alpha=0.05\left(D_{n, \alpha}=0.50, D_{\text {critical }}=0.38, n=13\right.$ storms). For the other three contaminants, the estimated effluent CDFs passed the K-S test for the same level of significance, which suggests that the calibrated model preserved the distribution of the observed effluent EMCs.

DIN

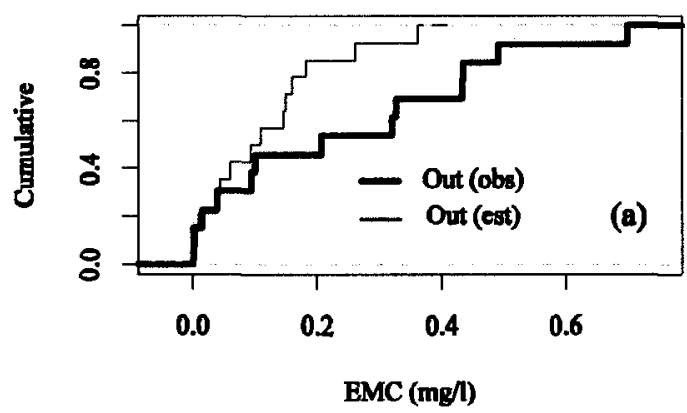

TSS

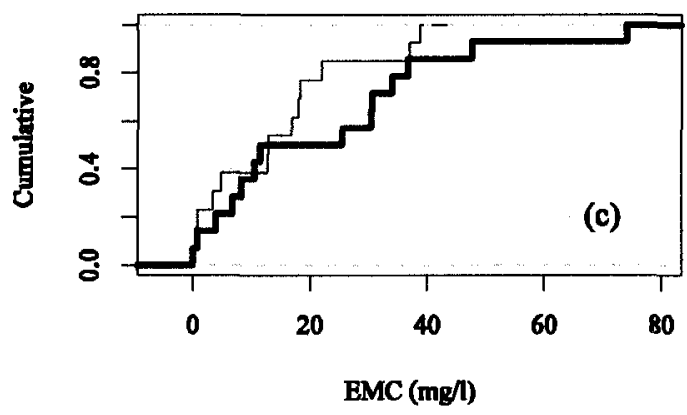

TPH-D

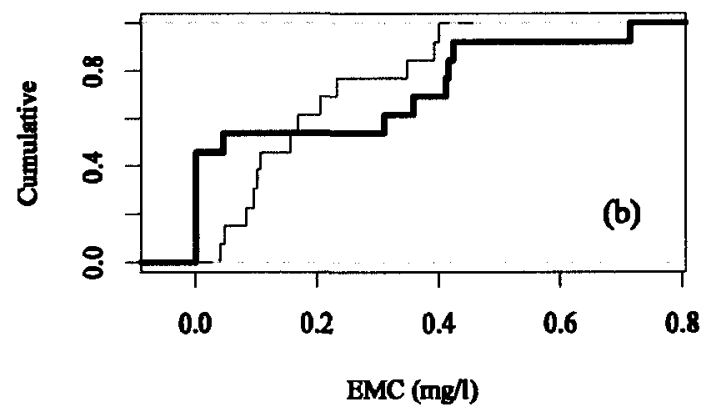

Zn

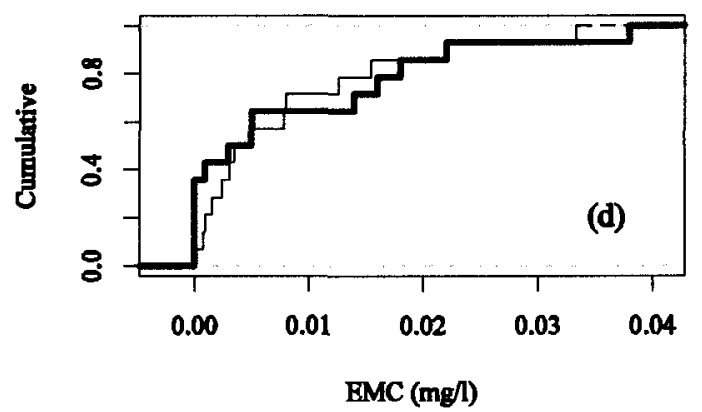

Figure 24 CDFs of Event Mean Concentrations (EMC) for the retention pond. Out: effluent; obs: observed; and est: estimated.

Figure 25 shows the computed CDFs for the sand filter. The estimated effluent CDF for $\mathrm{Zn}, \mathrm{TPH}-\mathrm{D}$, and DIN passed the K-S test. The estimated effluent CDF for TSS did not pass the K-S test for a level of significance $\alpha=0.05\left(D_{n, \alpha}=0.58, D_{\text {critical }}=0.39, n=12\right.$ storms). This suggests that the model underestimates effluent EMCs for TSS. 
DIN

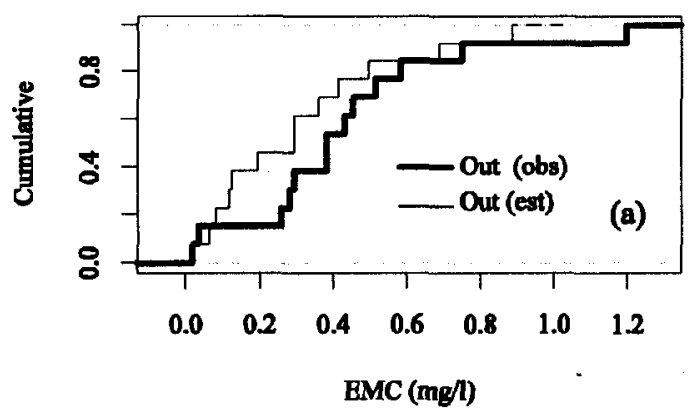

TSS

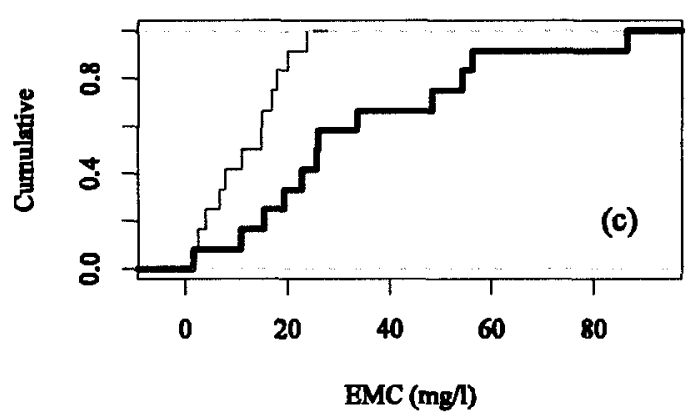

TPH-D

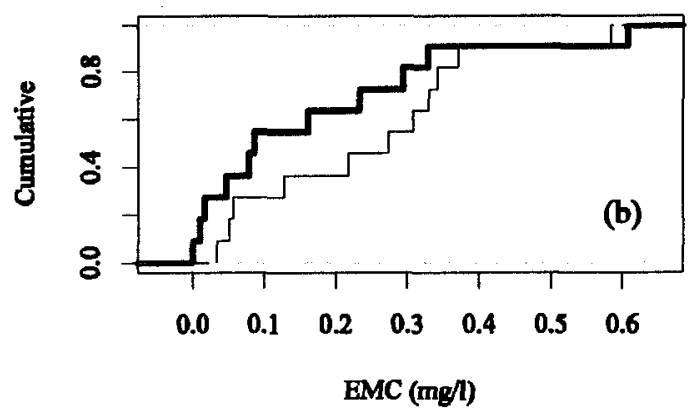

Zn

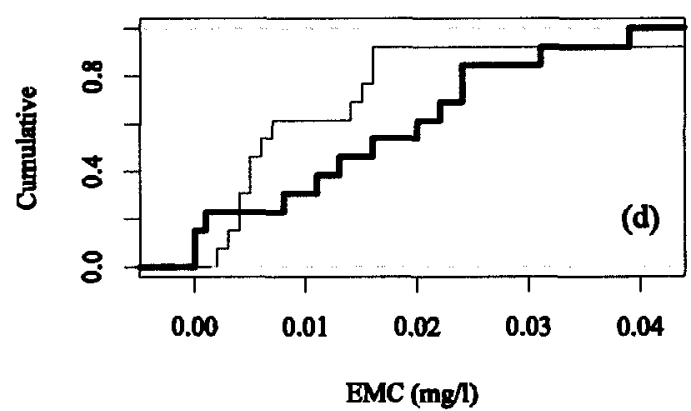

Figure 25 CDFs of Event Mean Concentrations (EMC) for the sand filter. Out: effluent; obs: observed; and est: estimated.

\section{Monte Carlo Simulation}

Model validation was performed using the Monte Carlo technique. For each stormwater system, one monitored storm event was not included (randomly selected) in the calibration stage so that effluent concentrations could be predicted using the statistical results. The 07/22/2006 storm event was used for the gravel wetland, the 06/01/2006 storm for the retention pond, and the 05/02/2006 storm for the sand filter. In 
this paper, only results for the gravel wetland and the retention pond are shown. Simulation results for the sand filter can be found in Avellaneda et al. (2008b).

For each Monte Carlo simulation, (k) was generated from the fitted gamma distributions and $\mathrm{n}$ was selected as the most frequent value observed after calibration. $\mathrm{A}$ total of 5,000 simulations were performed for each storm event and contaminant. Figure 26 and 27 show simulation results for the gravel wetland and the retention pond, respectively. The central solid line indicates the expected mean concentrations. The 10 $\left(q_{10}\right), 30\left(q_{30}\right), 70\left(q_{70}\right)$, and $90\left(q_{90}\right)$ percentiles were selected as a measure of uncertainty.

Figure 26 shows Monte Carlo simulation results for the gravel wetland. A decay order was selected for each contaminant: $n=2$ for TSS, $n=2$ for TPH-D, $n=1$ for $Z n$, and $n=1$ for DIN. A total of four samples were analyzed during this storm. The majority of the samples were reported with concentrations values below detection limit: $0.05 \mathrm{mg} / \mathrm{l}$ for DIN, $0.5 \mathrm{mg} / \mathrm{l}$ for TPH-D, $10 \mathrm{mg} / \mathrm{l}$ for TSS, and $0.01 \mathrm{mg} / \mathrm{l}$ for $\mathrm{Zn}$. Only for the first sample, the concentration value was above the detection limit for TSS and TPH-D. For all the contaminants, the model predicted well the concentrations below and above the detection limit. 

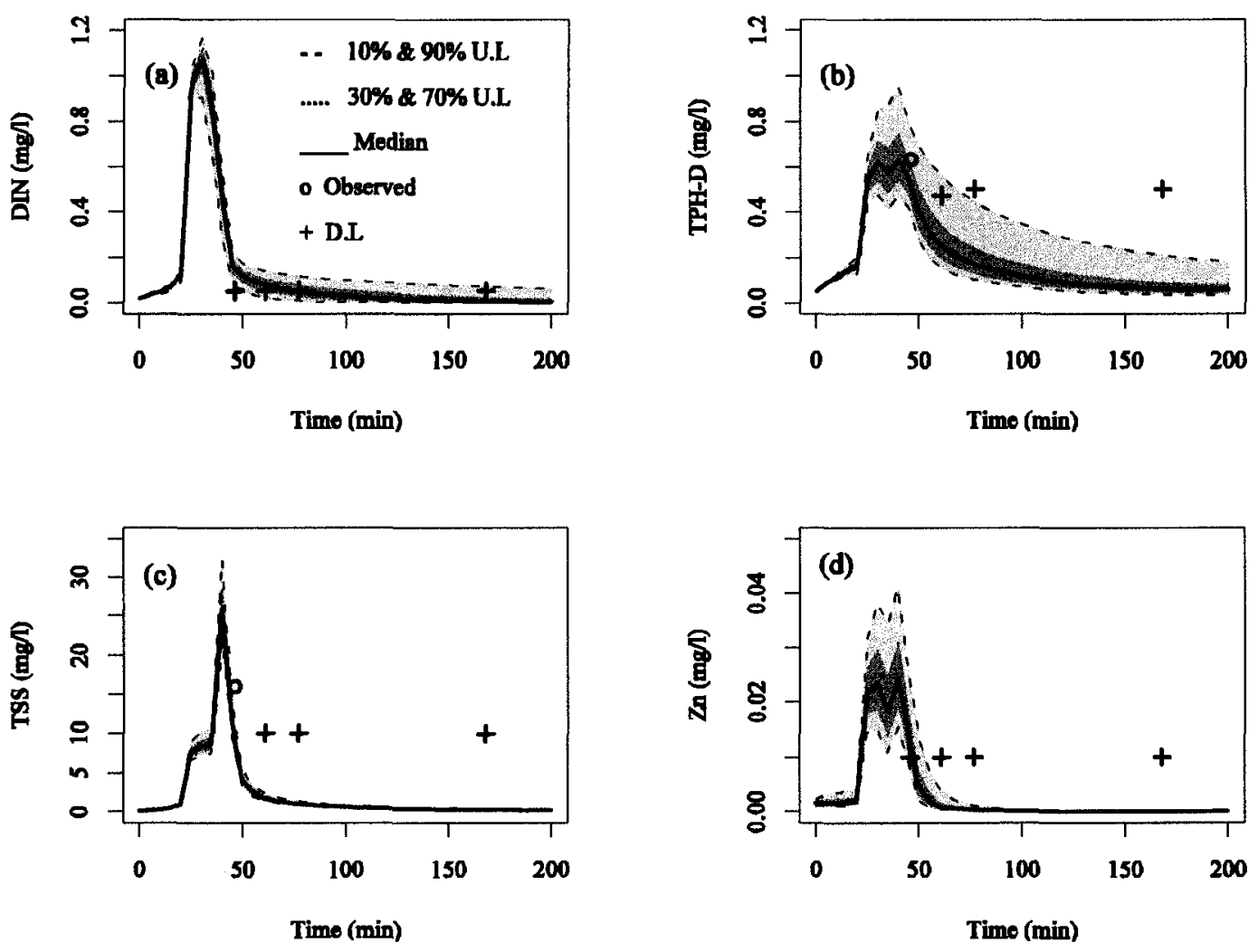

Figure 26 Gravel wetland Monte Carlo simulation results for the 07/22/2006 storm. U.L: Uncertainty limits. The dark shaded region indicates the $30 \%$ and $70 \%$ uncertainty limits. The light grey shaded region indicates the $10 \%$ and $90 \%$ uncertainty limits. Observed concentrations values and the reported detection limits were plotted as discrete points.

Figure 27 shows Monte Carlo simulation results for the retention pond. The following decay orders were selected: $n=2$ for TSS, $n=2$ for TPH-D, $n=1$ for $Z n$, and $n=2$ for DIN. For this storm, concentrations below the detection limit were reported only for TPHD. The model underestimated effluent concentrations for DIN. It should be noticed that the poor results for DIN could be due to the fact that a good fit of the gamma distribution for (k) was not achieved. For TPH-D, simulated effluent concentrations fell below the reported detection limit. Observed effluent TSS concentrations fell within the $30 \%$ and $70 \%$ uncertainty limits (except for the first sample) provided by the Monte Carlo 
simulations. The model tended to underestimate effluent $\mathrm{Zn}$ concentrations; however, observed concentrations did fall within the $10 \%$ and $90 \%$ uncertainty limits.
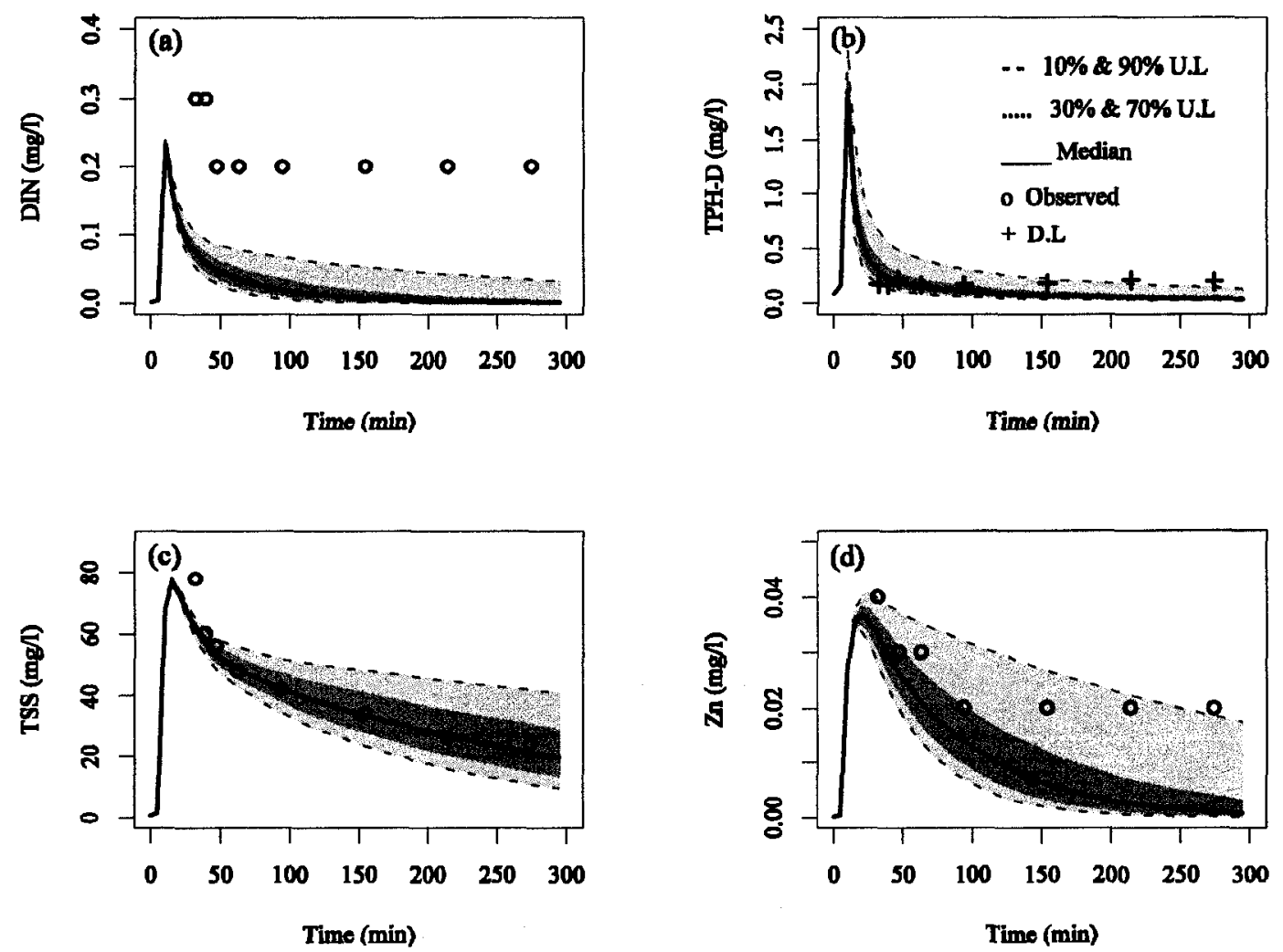

Figure 27 Retention pond Monte Carlo simulation results for the 06/01/2006 storm. U.L: Uncertainty limits. The dark shaded region indicates the $30 \%$ and $70 \%$ uncertainty limits. The light grey shaded region indicates the $10 \%$ and $90 \%$ uncertainty limits. Observed concentrations values and the reported detection limits were plotted as discrete points.

Table 21 shows effluent observed and estimated total effluent mass for the 06/01/2006 storm for the retention pond (RP) and 07/22/2006 storm for the gravel wetland (GW). The observed mass was computed using two different methods when a detection limit was reported: (1) using a zero concentration and (2) generating a concentration from the probability distributions fitted to the effluent data (Table 17). The 
three estimated values correspond to the $30 \%\left(q_{30}\right), 50 \%\left(q_{50}\right)$, and $70 \%\left(q_{70}\right)$ uncertainty limits. The computed uncertainty limits were smaller than the observed effluent DIN and Zn mass for the 06/01/2006 storm. However, for this storm, the observed effluent TSS mass fell within the estimated uncertainty limits. For Zinc, the median estimated mass was $2.27 \mathrm{~g}$ and the observed mass was $0 \mathrm{~g}$ using method (1) and $6.89 \mathrm{~g}$ using method (2). For the $07 / 22 / 2006$ storm, the median estimated effluent mass was higher than the observed effluent mass for all the contaminant when method (1) was used. However, the observed effluent mass was higher than $q_{70}$ for all the contaminants when method (2) was used.

Table 21 Observed and estimated total effluent mass for the storms used for validation.

\begin{tabular}{ccccccc}
\hline \multirow{2}{*}{ Storm } & \multirow{2}{*}{ Pollutant } & \multicolumn{5}{c}{ Total Mass (g) } \\
\cline { 3 - 7 } & & Observed (1) & Observed (2) & Estimated $\left(q_{30}\right)$ & Estimated $\left(q_{50}\right)$ & Estimated $\left(q_{70}\right)$ \\
\hline $7 / 22 / 2006(\mathrm{GW})$ & TSS & 8 & 74 & 11 & 12 & 13 \\
& TPH-D & 0.32 & 2.42 & 1.02 & 1.30 & 1.76 \\
& Zn & 0.00 & 0.01 & 0.007 & 0.010 & 0.014 \\
& DIN & 0.00 & 2.92 & 0.20 & 0.32 & 0.51 \\
$6 / 1 / 2006$ (RP) & TSS & 817 & 817 & 703 & 812 & 955 \\
& TPH-D & 0.00 & 6.89 & 1.60 & 2.27 & 3.25 \\
& Zn & 0.49 & 0.49 & 0.17 & 0.23 & 0.34 \\
& DIN & 4.58 & 4.58 & 0.25 & 0.38 & 0.62 \\
\hline
\end{tabular}

(1) A zero concentration was used when a DL (detection limit) was reported.

(2) Concentrations were determined from a fitted gamma probabiliy distribution (Table 10) when a DL was reported.

GW: Gravel Wetland. RP: Retention Pond.

\section{Conclusions}

The calibration of an n-order decay model to estimate effluent concentrations from a gravel wetland, a retention pond, and a sand filter was presented. The optimum 
combination of the removal rate $(k)$ and the decay order $(n)$ was found on storm-bystorm basis for each stormwater system. The variability of the parameters was summarized by using probability distribution functions. In particular, a gamma distribution was found to best represent the distribution of the removal rate. A zero, a first, and a second order decay model were investigated in this study. The simulated annealing method was used as the optimization technique and the sum-of-squares estimator was adopted as the objective function.

A comparison of the median removal rate among the different systems was made. The highest median DIN removal rate was found for the gravel wetland. The DIN removal rate was lower for the retention pond and the sand filter. The median TPH-D removal rate was similar for all the systems. The gravel wetland exhibited the highest TSS removal rate whereas lower and similar median removal rates were obtained for the other two systems. Note that a multivariate analysis of variance was not performed due to the small size of the data set and the possibility that the distributions did not follow a normal distribution.

It was found that first and second order decay models were likely to describe pollutant treatment for the different contaminants. A descriptive statement of the variability of the decay order was made by analyzing the calibrated values. Second-order decay models were found to best describe effluent pollutant concentrations for TSS and TPH-D. For $\mathrm{Zn}$, a first-order decay model was frequently found as the optimum. Model results suggested that both a first and a second order decay model were likely to describe DIN removal.

The effluent EMC was estimated and compared against the observed effluent EMC for the storms used for calibration. For this purpose, the similarity between the distribution of the estimated effluent EMCs and the observed effluent EMCs was tested. It was found that for most of the contaminants and systems, the calibrated model was 
able to reproduce the distribution of the observed data. This criterion is recommended when assessing the quality of the calibrated model. This procedure could not be performed on the gravel wetland due to the fact that a high number of the effluent samples were reported with values below the detection limit, which ended up affecting the computation of the EMC.

An effort to validate the calibrated model was made by performing Monte Carlo simulations using the statistical information obtained for each parameter. As an example, effluent concentrations were found for two storms not included in the calibration of the model. Removal rate values were generated from the fitted gamma distribution for each contaminant and the decay order was selected as the most likely approach between a first and a second order model. Most of the observed effluent concentrations fell within the uncertainty limits provided by the Monte Carlo simulations; although, poor results were obtained for the effluent DIN concentrations of the retention pond. Further investigations should assess the performance of the model using a large set of storms. 


\title{
CHAPTER 5
}

\section{A BAYESIAN STORMWATER QUALITY MODEL AND ITS APPLICATION TO WATER QUALITY MONITORING}

\begin{abstract}
A Bayesian statistical approach for determining parameter uncertainty of a stormwater treatment model is presented. Pollutant removal is simulated by using an norder decay model in which the removal rate $(k)$ and the decay order $(n)$ are the parameters. The ultimate goal was to determine the posterior probability distribution of the removal rate parameter for a selected group of stormwater treatment technologies and contaminants. The stormwater treatment technologies included: a sand filter, a gravel wetland, and a retention pond; all systems were loaded and monitored in a side by side fashion over a two year period. Stormwater runoff was generated by ambient rainfall on a commuter parking lot located at the University of New Hampshire. Results are presented for the following pollutants of concern: total suspended solids (TSS), total petroleum hydrocarbons - diesel range hydrocarbons (TPH-D), dissolved inorganic nitrogen (DIN, comprised of nitrate, nitrite, and ammonia), and Zinc ( $\mathrm{Zn}$ ). The targeted posterior distributions were numerically estimated by using a conventional Metropolis Hastings algorithm. It was found that first and second order decay models were more likely to reproduce estimated effluent concentrations. For a second order decay model, mean removal rate values were computed from the posterior distributions. Specifically, for the gravel wetland: $k_{T S S}=59, k_{Z_{n}}=2115, k_{T P H-D}=88, k_{D I N}=7$; for the sand filter: $k_{T S S}=$
\end{abstract}


$1.7, \mathrm{k}_{\mathrm{Zn}}=1568, \mathrm{k}_{\mathrm{TPH}-\mathrm{D}}=57, \mathrm{k}_{\mathrm{DIN}}=2 ;$ and for the retention pond: $\mathrm{k}_{\mathrm{TSS}}=0.8, \mathrm{k}_{\mathrm{Zn}}=4645$, $\mathrm{k}_{\mathrm{TPH}-\mathrm{D}}=68, \mathrm{k}_{\mathrm{DIN}}=8\left(\mathrm{k}\right.$ in units of $(\mathrm{mg} / \mathrm{l})^{-1} /$ day $)$.

\section{Introduction}

Environmental models are developed, in general, to capture the complexity of a given physical problem and to conceptualize its features in mathematical expressions that describe the system dynamics. Normally, these mathematical expressions relate relevant environmental variables and some fitting parameters. The purpose of model calibration is then to find the best set of fitting parameters using field observations. However, due to the complexity of natural systems and the limitations on model components and characteristics, calibration may be difficult to achieve if not impossible. Furthermore, more than one set of parameters may describe the observed data. For that reason, parameter uncertainties must be considered when calibrating a model (Beven and Binley, 1992). Modelers turn to Bayesian inference when estimation of parameter uncertainties is necessary.

Environmental Bayesian models have been formulated for a variety of applications: calibration and prediction in conceptual rainfall-runoff modelling (Kuzzera et al., 2006), contaminant fate and transport in surface waters (Steinberg et al., 1996; Hong et al., 2005), flood frequency analysis using historical data (Reis and Stedinger, 2005; Seidou et al., 2006), and analysis of extreme values (Renard et al., 2006). In these studies, the objectives were to identify parameter values, evaluate the model structure, and state variable prediction. Additionally, Bayesian statistics provided a model for assessing parameter uncertainty.

Some stormwater quality models have been developed to estimate pollutant concentrations at the outlet of a watershed. Flow and water quality information are 
commonly used as the input variables so that parameters such as the accumulation rate and the wash-off coefficient are to be estimated. Kanso et al. (2003) studied parameter uncertainties of an urban stormwater model using a classical formulation for the transport of contaminants (the so-called accumulation and wash-off model). This study provided posterior probability distributions (PPDs) for the parameters of the model. Gaume et al. (1998) performed an uncertainty analysis using the Storm Water Management Model (SWMM). In their study, an accumulation and wash-off model was calibrated using a selected group of storms. Additionally, the study showed that even though a good fit between estimated and observed values was achieved, validation was still a challenge due to large uncertainties associated with the parameters. Other studies have used different statistical techniques to measure model performance on stormwater applications (Arabi et al, 2007; Walker, 1994).

Very few applications have been implemented to describe parameter variations for models describing pollutant removal from stormwater management technologies. An norder decay model for simulating this type of treatment was applied by Avellaneda et al. (2008b, 2008c) on various systems, each receiving the same stormwater influent characteristics. Each system was individually monitored for a variety of contaminants. Calibrated parameter values were obtained on a storm by storm basis by minimizing the error function, which was defined as the sum of the squares of the residuals between the observed and estimated concentrations. Although a mechanistic approach was described in this research, this study suggested the application of more advanced statistical tools to explore parameter variation and uncertainty of estimated values.

In this paper, a Bayesian statistical approach was used to estimate parameter values for a stormwater quality model. This was accomplished for an n-order decay model. The parameters of the model were the removal rate $(k)$ and the decay order $(n)$. The objectives were: (i) to calibrate a Bayesian stormwater quality model for three 
stormwater systems (a gravel wetland, a sand filter, and a retention pond) using a collection of monitored rainfall events; (ii) to determine the posterior probability distribution (PPD) of the removal rate $k$ for different decay order values $(n=0,1,2)$; and (iii) to perform Monte Carlo simulations to estimate effluent pollutant concentrations from the stormwater systems using the calibrated model. The contaminants of interest were: total suspended solids (TSS), total petroleum hydrocarbons - diesel range hydrocarbons (TPH-D), dissolved inorganic nitrogen (DIN, comprised of nitrate, nitrite, and ammonia), and zinc $(\mathrm{Zn})$. Monitored data consisted of influent flow, effluent flow, and the analytical results water quality samples collected for storm events between the 2004 and 2006 period.

\section{Stormwater Treatment Measures}

Three stormwater management measures were selected for this study: a retention pond, a sand filter, and a gravel wetland. The design parameters were taken from the New York State Stormwater Management Design Manual (2001). A pretreatment sedimentation basin was constructed for each system in order to help remove large particles, provides from some flow equalization, and prevents the systems from premature clogging. The designed Water Quality Volume was $92.5 \mathrm{~m}^{3}$ (WQV), which represents $25 \mathrm{~mm}$ of precipitation over an impervious area of $4047 \mathrm{~m}^{2}$, and corresponds to the daily storm volume not exceeded $90 \%$ of the time on days with measurable precipitation. 


\section{Gravel Wetland}

The gravel wetland is considered an infiltration / filtration system. The system is comprised of a sedimentation forebay and two horizontal-flow treatment cells. The filter media is a combination of a gravel substrate at the bottom $\left(0.6 \mathrm{~m}\right.$ thick and $D_{50}=19$ $\mathrm{mm}$ ) and a wetland soil on top. A $0.15 \mathrm{~m}$ perforated pipe is used to distribute the influent flow across the width of the subsurface gravel bed. Basic areal dimensions of the studied gravel wetland are $4.6 \mathrm{~m} \times 9.8 \mathrm{~m}$ for the filter basin footprint (for each cell) and $11.3 \mathrm{~m} \times 17.1 \mathrm{~m}$ for the forebay footprint. Stormwater flows horizontally through the gravel substrate and is collected by subdrains on the far end of the second treatment cell. The forebay was designed to hold $10 \%$ of the WQV while each treatment cell holds $45 \%$. The total area of the gravel wetland is $507 \mathrm{~m}^{2}$. Contaminants are removed by filtration, biological uptake, and adsorption.

\section{Retention Pond}

Retention ponds are designed to retain a large volume of stormwater and slowly release it. Treatment is achieved by physical settling of sediment and biological uptake. The areal dimensions of the studied retention pond are $14 \mathrm{~m} \times 21 \mathrm{~m}$. The treated runoff leaves the system through a $0.15 \mathrm{~m}$ perforated standpipe with reducing coupling to $0.025 \mathrm{~m}$. The surface area of the retention pond is covered by algae or aquatic plants. Side slopes were stabilized with grass, and spillways with stone and geotextile. 


\section{Sand Filter}

The sand filter bed is $0.6 \mathrm{~m}$ thick, uses coarse to medium grain sand $\left(D_{50}=0.7 \mathrm{~mm}\right)$, and has areal dimensions of $2.4 \mathrm{~m} \times 6.1 \mathrm{~m}$. Temporary ponding of the filter basin is expected during larger storm events due to saturation of the filter media and the fact that inflow exceeds outflow. The sedimentation forebay was designed to hold $25 \%$ of the WQV while the filter basin holds the remaining $75 \%$.The filter bed is sub-drained by a $0.15 \mathrm{~m}$ perforated pipe bedded in a $0.20 \mathrm{~m}$ layer of crushed stone $\left(D_{50}=19 \mathrm{~mm}\right)$.

\section{Monitoring}

Influent and effluent for these three stormwater treatment measures were monitored between August 2004 and September 2006. A total of 15 storms were collected for the gravel wetland, 15 storms for the retention pond, and 16 storms for the sand filter. Automated samplers (6712SR ISCO) performed the sampling. Normally 8-12 samples per storm event were used to characterize both influent and effluent stormwater quality. The sampling program for each system was based on analyses of typical effluent hydrographs. Influent and effluent flows were measured with inline weirs.

Runoff constituent analysis included: total suspended solids (TSS), total petroleum hydrocarbons - diesel range hydrocarbons (TPH-D), dissolved inorganic nitrogen (DIN, comprised of nitrate, nitrite, and ammonia), and zinc ( $\mathrm{Zn})$. Stormwater samples were analyzed by a laboratory that is state-certified for drinking water and wastewater. Guidance documents on collecting environmental data and the site quality assurance project plan were followed. Table 22 shows characteristics of the storm events selected for this study. Note that the storm characteristics were obtained for the total watershed area; however, each system treated an equivalent fraction of the total storm volume. 
Additionally, not always the three systems were monitored simultaneously for all the storm events. More description about the monitoring program is presented in Roseen et al. (2006).

Tabie 22 Summary of monitored storm events.

\begin{tabular}{|c|c|c|c|c|c|c|c|}
\hline $\begin{array}{c}\text { Rainfall } \\
\text { Event } \\
(\mathrm{m} / \mathrm{d} / \mathrm{y})\end{array}$ & $\begin{array}{c}\text { Peak } \\
\text { Intensity } \\
\text { (mm/hr) }\end{array}$ & $\begin{array}{c}\text { Rain } \\
\text { Duration } \\
\text { (min) }\end{array}$ & $\begin{array}{l}\text { Total } \\
\text { Depth } \\
(\mathrm{mm})\end{array}$ & $\begin{array}{c}\text { Peak } \\
\text { Flow } \\
\text { (m²/day) }\end{array}$ & $\begin{array}{c}\text { Volume } \\
\left(\mathrm{m}^{3}\right)\end{array}$ & $\begin{array}{c}\text { Anticedent } \\
\text { Dry Period } \\
\text { (days) }\end{array}$ & Season \\
\hline $9 / 18 / 2004$ & 15 & 1075 & 50 & 5642 & 1364 & 7.0 & Fall \\
\hline $10 / 30 / 2004$ & 21 & 705 & 11 & 8678 & 281 & 13.0 & Fall \\
\hline $11 / 24 / 2004$ & 9 & 705 & 18 & 4394 & 530 & 3.5 & Fall \\
\hline $1 / 14 / 2005$ & 24 & 645 & 17 & 21101 & 1033 & 1.3 & Winter \\
\hline $2 / 10 / 2005$ & 6 & 1520 & 32 & 4437 & 795 & 3.6 & Winter \\
\hline $3 / 8 / 2005$ & 3 & 1220 & 20 & 2338 & 406 & 5.7 & Winter \\
\hline $3 / 28 / 2005$ & 12 & 1685 & 60 & 7675 & 3082 & 3.4 & Winter \\
\hline $4 / 20 / 2005$ & 12 & 480 & 15 & 4274 & 1017 & 5.9 & Spring \\
\hline $6 / 22 / 2005$ & 15 & 95 & 8 & 9120 & 266 & 4.0 & Summer \\
\hline $8 / 13 / 2005$ & 24 & 765 & 13 & 18408 & 514 & 10.0 & Summer \\
\hline $9 / 15 / 2005$ & 18 & 30 & 5 & 5518 & 86 & 10.0 & Fall \\
\hline $11 / 6 / 2005$ & 12 & 100 & 7 & 2801 & 135 & 10.8 & Fall \\
\hline $11 / 30 / 2005$ & 9 & 810 & 18 & 2503 & 363 & 5.0 & Fall \\
\hline $12 / 16 / 2005$ & 18 & 630 & 35 & 2561 & 458 & 5.5 & Winter \\
\hline $1 / 11 / 2006$ & 15 & 320 & 15 & 2903 & 214 & 5.8 & Winter \\
\hline $2 / 17 / 2006$ & 12 & 110 & 3 & 1493 & 37 & 2.5 & Winter \\
\hline $3 / 13 / 2006$ & 12 & 170 & 7 & 1345 & 75 & 2.5 & Winter \\
\hline $5 / 2 / 2006^{*}$ & 12 & 1920 & 60 & 4642 & 1331 & 7.0 & Spring \\
\hline $5 / 9 / 2006$ & 3 & 565 & 14 & 1621 & 322 & 5.6 & Spring \\
\hline $6 / 1 / 2006$ & 125 & 485 & 51 & 27194 & 930 & 10.7 & Summer \\
\hline $6 / 21 / 2006$ & 27 & 80 & 5 & 5190 & 93 & 4.7 & Summer \\
\hline $7 / 22 / 2006^{*}$ & 40 & 50 & 5 & 8333 & 81 & 7.5 & Summer \\
\hline $9 / 6 / 2006$ & 30 & 585 & 16 & 6087 & 410 & 4.5 & Fall \\
\hline
\end{tabular}

"Storms used for validation 


\section{Model Structure}

\section{Stormwater Treatment Model}

An n-order decay model was implemented to estimate effluent pollutant concentrations from the stormwater treatment systems. The parameters of the model were the removal rate $(k)$ and the decay order $(n)$. The treatment system was conceptualized as a black box where influent and effluent variables must be determined or measured (Avellaneda et al., 2008b). The partial differential equation that describes time variations of effluent pollutant concentrations is:

$$
\frac{d C_{\text {out }}}{d t}=\frac{C_{\text {in }} Q_{\text {in }}-C_{\text {out }} Q_{\text {out }}}{S}-\frac{C_{\text {out }}}{S} \frac{d S}{d t}-k C_{\text {out }}^{n}
$$

where $C_{\text {out }}$ represents the effluent concentration $(\mathrm{mg} / \mathrm{l}) ; \mathrm{C}_{\text {in }}$ is the influent concentration $(\mathrm{mg} / \mathrm{l}) ; \mathrm{Q}_{\text {in }}$ is the influent flow rate $\left(\mathrm{m}^{3} /\right.$ day); $Q_{\text {out }}$ is the effluent flow rate $\left(\mathrm{m}^{3} /\right.$ day); $S$ is the stored volume within the system $\left(\mathrm{m}^{3}\right) ; \mathrm{dS} / \mathrm{dt}$ is the change in stored volume $\left(\mathrm{m}^{3} /\right.$ day); $t$ is time (days); $k$ is the removal rate $\left((\mathrm{mg} / \mathrm{l})^{-\mathrm{n}+1} /\right.$ day); and $\mathrm{n}$ is the decay order $(-)$. These units will be used throughout the paper. For more details about the model see Avellaneda et al. $(2008 b, 2008 c)$.

\section{Bayesian Parameter Estimation}

A conceptual model is necessary to establish a relationship between observed and estimated values. A nonlinear regression model can be used to define such relationship (Marshall et al., 2004; Kanso et al., 2003; Bates and Campell, 2001). Normally, the observed values are set equal to the estimated values plus a residual term as follows: 


$$
C_{\text {out }, \text { obs }}^{i}=C_{\text {out }, \text { est }}^{i}\left(x^{i}, \Theta\right)+\varepsilon^{i} \quad i=1, \ldots, m
$$

where $\mathrm{C}_{\text {out,obs }}$ represents the observed effluent concentrations; $\mathrm{C}_{\text {out,est }}$ is the corresponding estimated effluent concentrations (Equation (15)); $i$ denotes the current sample; a total number of samples $m$; $x$ is the set of input variables (influent flow, influent concentrations, effluent flow, storage, and change in storage); $\Theta$ represents the parameter set ( $k$ and $n)$; and $\varepsilon$ is a residual term. Further details on the residual term are provided later in this section.

The Bayesian paradigm provides a framework for estimating model parameters using pre-existing knowledge about the parameters and observed information. The preexisting knowledge about the model parameters is summarized in the prior probability distribution or, $P(\Theta)$, for each parameter. In many cases, the prior distribution is given a large variance or a uniform distribution, reflecting the lack of knowledge about the parameter. A prior distribution can also be determined from previous studies or expert knowledge. After performing the Bayesian update, the current state of the parameter values is summarized in the posterior probability distribution (PPD).

The PPD is obtained from Bayes' theorem, which states that the current state of the parameters is proportional to the likelihood function multiplied by the prior density. The PPD can be written as follows:

$$
P\left(\Theta \mid C_{\text {out }, \text { obs }}\right)=\frac{P\left(C_{\text {out obs }} \mid \Theta\right) P(\Theta)}{P\left(C_{\text {out }, \text { obs }}\right)}=\frac{P\left(C_{\text {out obs }} \mid \Theta\right) P(\Theta)}{\int P\left(C_{\text {out }, \text { obs }} \mid \Theta\right) P(\Theta) d \Theta}
$$


where: $P\left(\Theta \mid C_{\text {out,obs }}\right)$ is the posterior probability distribution PPD of the model parameters; $P(\Theta)$ is the prior distribution of the model parameters; $P\left(C_{\text {out,obs }} \mid \Theta\right)$ is the likelihood function; and $\mathrm{P}\left(\mathrm{C}_{\text {out,obs }}\right)$ is a normalizing factor that represents the probability of obtaining the observed concentrations. For this study, a uniform probability distribution was used as the prior distribution for the removal rate parameter and appropriate bounds will be provided in the following sections. The posterior distribution assumes a shape similar to the prior when available data are limited. The posterior distribution is more influenced by the data when sufficient information is provided (Mashall et al., 2004).

The updating mechanism provided in equation (17) relies on the specification of the likelihood function $P\left(C_{\text {out,obs }} \mid \Theta\right)$. The likelihood function represents the probability of obtaining the observed concentrations given some parameter values. Normally, the likelihood is defined as a function of the residuals $\varepsilon^{\prime}$ (Beven and Binley, 1992). For this study, the residuals were assumed to be independent and identically distributed according to a normal distribution $\mathcal{N}\left(0, \sigma^{2}\right)$. Then, the likelihood function was defined as follows:

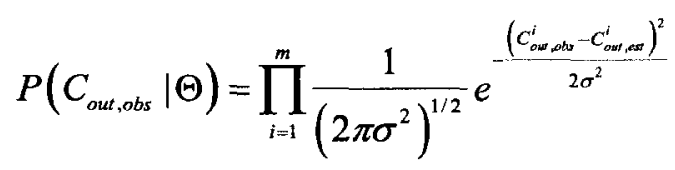

The error variance $\sigma^{2}$ was treated as a parameter of the Bayesian model. Therefore, a prior distribution for the error variance was defined by using an Inverse Gamma function with parameters $\alpha_{\mathrm{i} . \mathrm{g}}=0.01$ and $\beta_{\mathrm{i} . \mathrm{g}}=0.01$, which define a non-informative prior distribution. Non-informative prior distributions play a minimal role in the analysis and let the data define the posterior distribution. The full conditional posterior distribution for $\sigma^{2}$ can be calculated from the likelihood function: 


$$
\begin{aligned}
& \sigma^{2} \mid k, x \sim \operatorname{Inv-Gamma}\left(\alpha_{i . g}+\frac{m}{2}, \beta_{i . g}+\frac{S S E}{2}\right) \\
& S S E=\sum_{i=1}^{m}\left(C_{o u t, o b s}^{i}-C_{o u t, e s t}^{i}\right)^{2}
\end{aligned}
$$

In some cases it is difficult to obtain the posterior probability distribution by direct calculation of the equation (17). In that case, the Metropolis - Hastings algorithm is used to obtain a numerical solution.

\section{Metropolis - Hastings Algorithm}

The objective of the Metropolis algorithm is to draw samples from the posterior distribution of the model parameters. In general, an arbitrary set of parameter values are used to start the process and then a subsequent set of parameters are generated from a Markov chain that follows a certain rule (Hastings, 1970; Smith and Roberts, 1993). This rule usually compares the current likelihood value to the likelihood calculated from a new potential set of parameter values. New "candidates" for the posterior distribution are accepted according to the likelihood ratio:

$$
\alpha_{\text {ratio }}<\frac{P\left(C_{\text {out }, \text { obs }} \mid \Theta^{*}\right) P\left(\Theta^{*}\right) Q\left(\Theta^{*} \mid \Theta^{t}\right)}{P\left(C_{\text {out }, \text { obs }} \mid \Theta^{t}\right) P\left(\Theta^{t}\right) Q\left(\Theta^{t} \mid \Theta^{*}\right)}
$$

where $\alpha_{\text {ratio }}$ is drawn from a uniform distribution $U(0,1) ; \Theta{ }^{t}$ represents the current state of the parameters; and $\Theta^{\star}$ are the parameter values generated from a proposal distribution Q. If the proposed values $\Theta{ }^{*}$ are accepted, then $\Theta{ }^{t+1}=\Theta^{*}$. Note that values from the 
proposal distribution will be accepted if there is an increase in the likelihood function: $P\left(C_{\text {out,obs }} \mid \Theta^{*}\right)>P\left(C_{\text {out,obs }} \mid \Theta^{t}\right)$. If the likelihood function decreases then the proposed values will be accepted with $\alpha_{\text {ratio }}$ probability. For this study, a normal distribution $\mathcal{N}\left(\Theta^{t}, \sigma\right.$

$\left.{ }^{2}\right)$ centered at the current state of the parameter was selected as the proposal distribution. An acceptance rate must be defined to ensure convergence of the Markov chain. An acceptance rate $\left(a_{r}\right)$ between 30 and $70 \%$ is considered to work well (Bates and Campbell, 2001). Additionally, a selected number of simulations are discarded at the beginning of the process to ensure that the final posterior distribution does not depend on the initial parameter values.

\section{Results}

\section{Model Calibration}

A group of storm events was selected for calibration purposes for each stormwater system. A total of 14 storm events were selected for the gravel wetland; 14 events for the retention pond; and 15 for the sand filter. One storm event was left for model validation. The calibration phase was performed using all the information available for the storm events. Therefore, final results such as estimated effluent concentrations and posterior probability distributions are based on the overall behavior of the systems during the study period.

A classical accumulation and wash-off model was used to calculate the influent concentrations (Avellaneda et al., 2008a). Optimum parameter values were calculated by minimizing the sum of squared errors of observed and estimated concentrations. Parameter values included: the mass of pollutant per unit area on the parking lot surface 
$\left(\mathrm{M}_{\mathrm{m}}\right)$; the pollutant removal rate $\left(\mathrm{k}_{\mathrm{b}}\right)$; a wash-off coefficient $\left(\mathrm{k}_{\mathrm{w}}\right)$; and a wash-off exponent (w). As an example, Figure 28 shows the sand filter influent information for the 04/20/2005 storm. This information consisted of the monitored influent and effluent hydrograph and the influent pollutograph for each contaminant. Optimized parameter values are reported in Table 23.
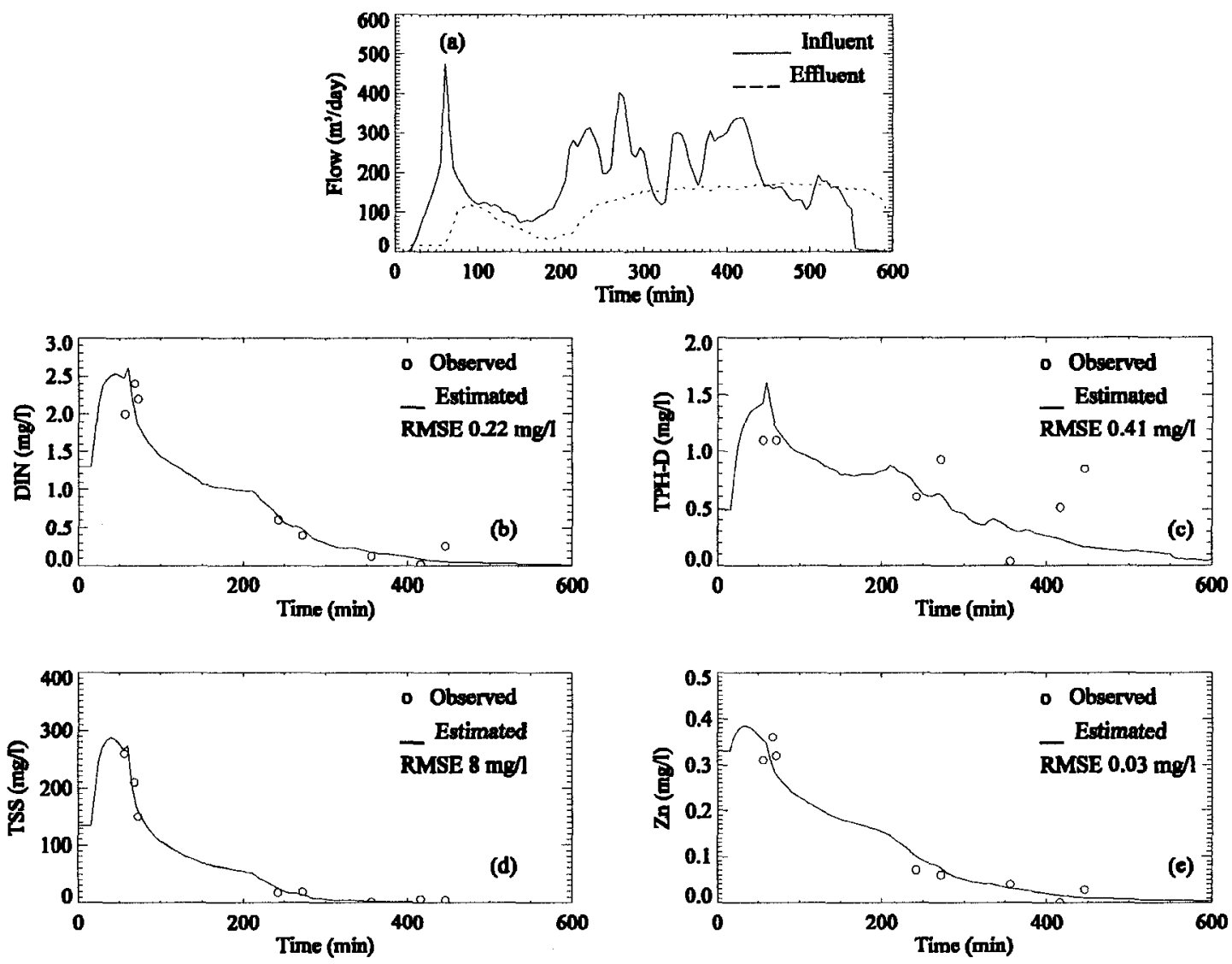

Figure 28 Sand filter influent information for the 04/20/2005 storm. 
Table 23 Optimized parameter values for the 04/20/2005 storm (sand filter).

\begin{tabular}{|c|c|c|c|c|}
\hline \multirow{2}{*}{ Pollutant } & \multicolumn{4}{|c|}{ Parameter } \\
\hline & $M_{m}\left[g / m^{2}\right]$ & $k_{b}\left[\right.$ day $\left.^{-1}\right]$ & $k_{w}[-]$ & $w[-]$ \\
\hline DIN & 0.194 & 0.010 & 0.003 & 1.13 \\
\hline TPH-D & 0.199 & 0.009 & 0.001 & 1.18 \\
\hline TSS & 1.73 & 0.098 & 0.004 & 1.15 \\
\hline $\mathrm{Zn}$ & 0.124 & 0.001 & 0.005 & 1.03 \\
\hline
\end{tabular}

Parameter estimation was performed for each system and contaminant. Equation (16) and an analytical solution for equation (15) were used to obtain the likelihood (18). Since the decay order $(n)$ was treated as a discrete value, it was decided to perform the analysis just for three scenarios: a zero, a first, and a second order decay model. It was necessary to establish some bounds for the removal rate (k) so a uniform prior distribution was defined. A uniform prior distribution was assumed due to the lack of knowledge about this parameter and the fact that only discrete optimum values may be found in the literature (for a first order day model see Wang et al., 2004; and Minton, 2002). Table 24 shows a range of values for each contaminant and system. These values were obtained from a previous study performed with the same database (Avellaneda et al., 2008c). A Gibbs sampler step (Gelman et al., 2004) was performed to generate values for the error variance $\sigma^{2}$ from the full conditional probability function (19). 
Table 24 Bounds of the removal rate $(\mathrm{k})$, which has units of $\left((\mathrm{mg} /)^{-n+1} / \mathrm{day}\right)$.

\begin{tabular}{lcccc}
\hline \multicolumn{1}{c}{ System } & TSS & Zn & TPH-D & DIN \\
\hline Gravel Wetland (GW) & $0-1000$ & $0-10000$ & $0-1000$ & $0-500$ \\
Sand Filter (SF) & $0-100$ & $0-10000$ & $0-1000$ & $0-100$ \\
Retention Pond (RP) & $0-100$ & $0-10000$ & $0-1000$ & $0-100$ \\
\hline
\end{tabular}

Examples of the computed posterior probability distributions (PPD) obtained via the Metropolis - Hastings algorithm are shown in Figures 29 to 30 . The parameter trace is displayed on top and the PPD for the removal rate $(k)$ and the error standard deviation $(\sigma)$ below. To compute the PPD, the first 1000 samples were discarded to allow for a "burn-in" period. The following 1000 samples were used to compute the PPD. In some cases, the number of iterations was increased (up to 2000) to ensure an acceptance rate $\left(a_{r}\right)$ between 0.3 and 0.7 . 

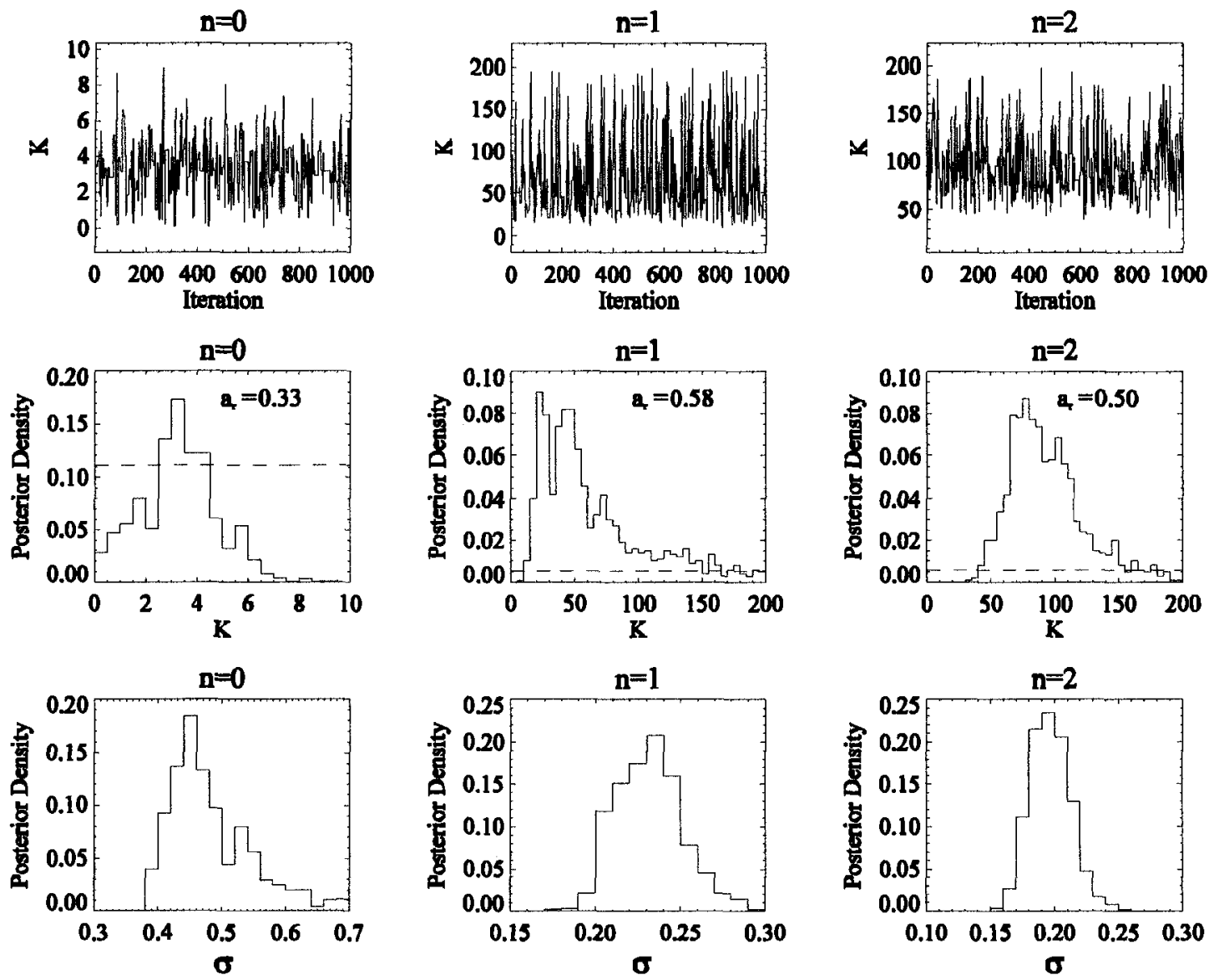

Figure 29 Parameter trace, PPDs (solid line), and prior distribution (dashed line) for the Gravel Wetland (TPH-D) and different decay orders (n). The removal rate (k) has units of $(\mathrm{mg} / \mathrm{l})^{-n+1} /$ day and the error standard deviation $(\sigma)$ units of $\mathrm{mg} / \mathrm{l}$. The acceptance rate $\left(\mathrm{a}_{\mathrm{r}}\right)$ is reported. 

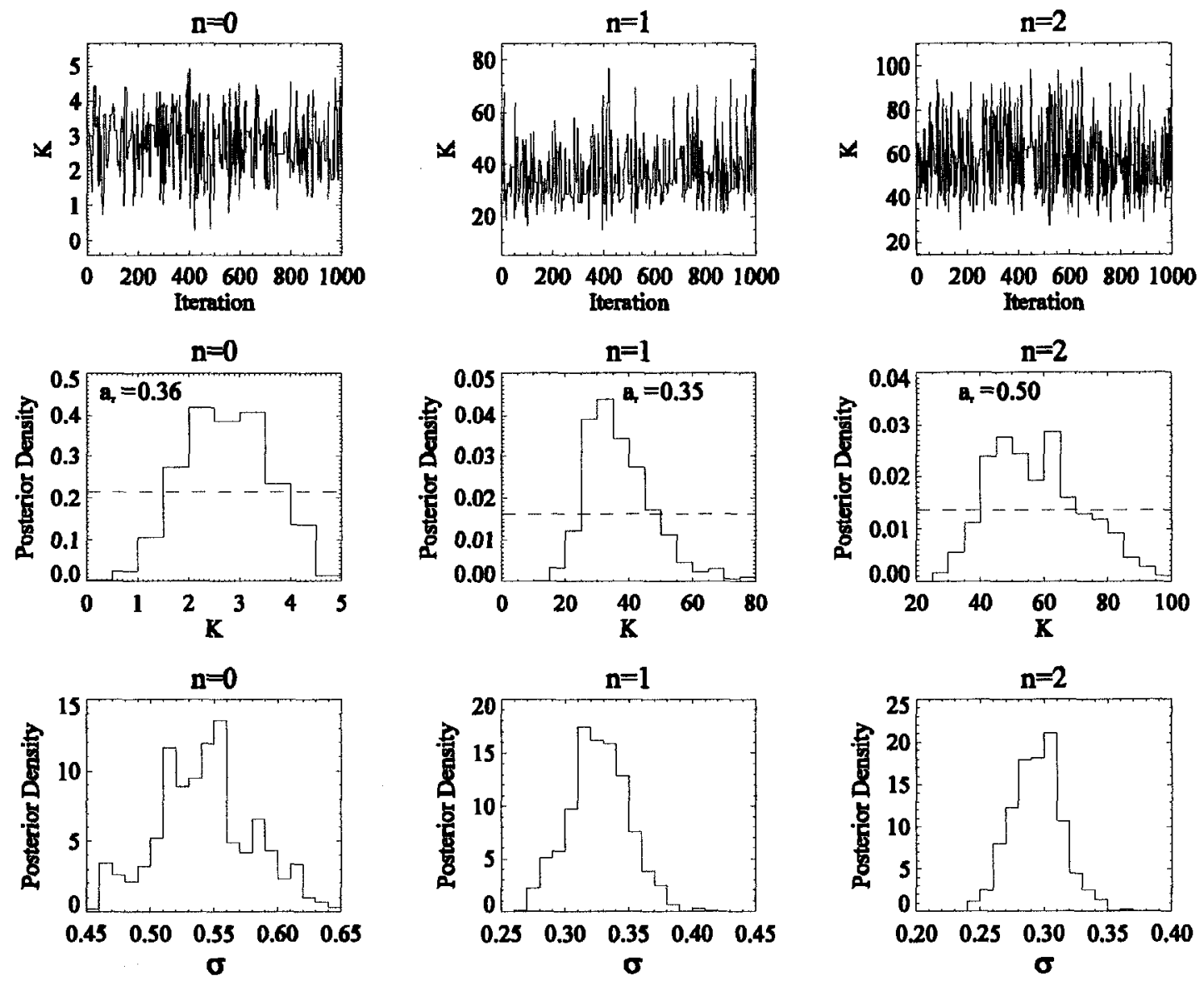

Figure 30 Parameter trace, PPDs (solid line), and prior distribution (dashed line) for the Sand Filter (TPH-D) and different decay orders $(n)$. The removal rate (k) has units of $(\mathrm{mg} / \mathrm{l})^{-n+1} /$ day and the error standard deviation $(\sigma)$ units of $\mathrm{mg} / \mathrm{l}$. The acceptance rate $\left(\mathrm{a}_{\mathrm{r}}\right)$ is reported. 

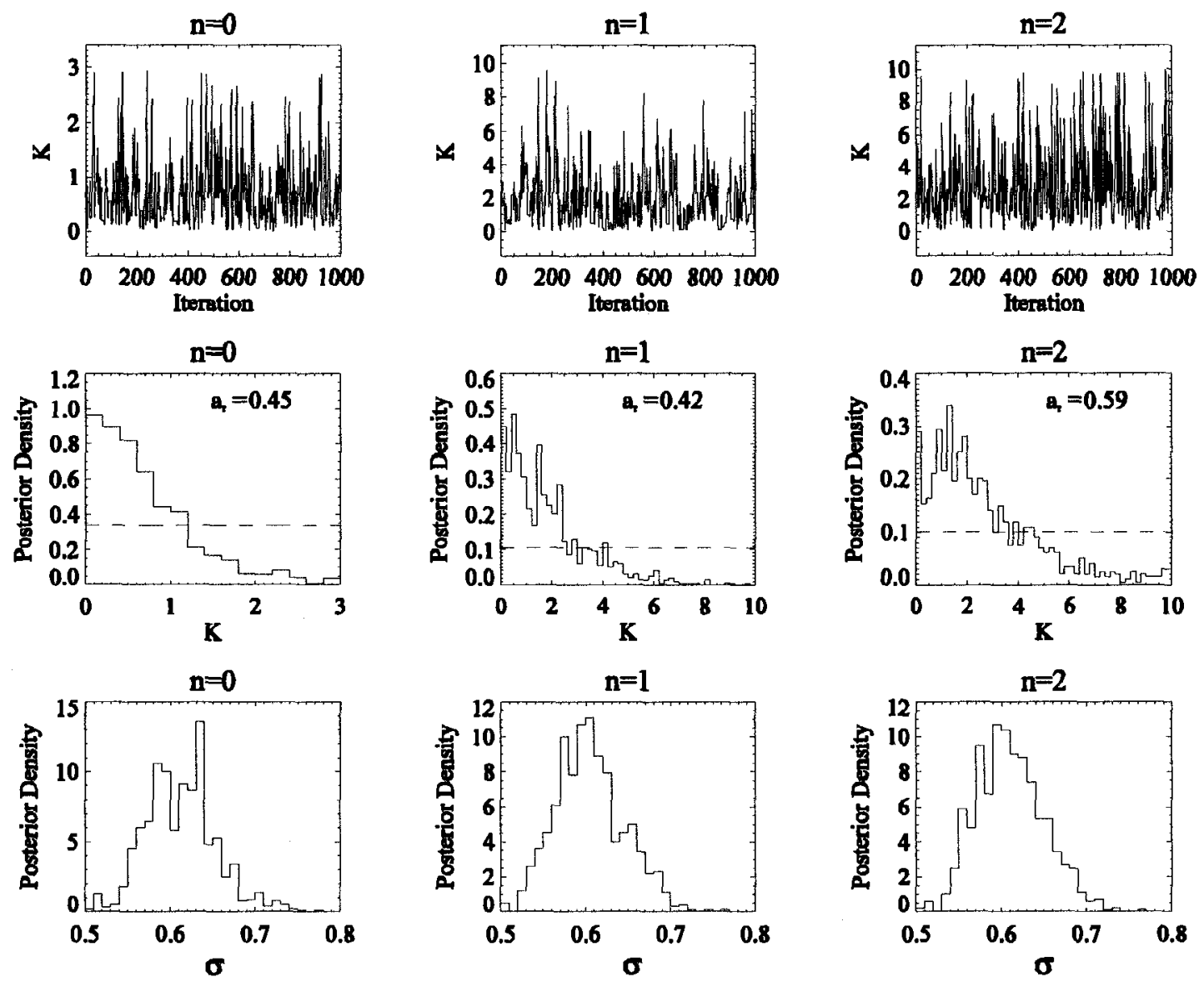

Figure 31 Parameter trace, PPDs (solid line), and prior distribution (dashed line) for the Sand Filter (DIN) and different decay orders $(n)$. The removal rate $(k)$ has units of $\left(\mathrm{mg} / \mathrm{l}^{-}\right.$ ${ }^{n+1} /$ day and the error standard deviation $(\sigma)$ units of $\mathrm{mg} / \mathrm{l}$. The acceptance rate $\left(\mathrm{a}_{\mathrm{r}}\right)$ is reported.

Posterior probability distributions were calculated for each system and contaminant using the previously described procedures. Table 25 summarizes the PPDs of the removal rate for a zero, a first, and a second order decay model for each system. Table 26 summarizes the PPDs of the error standard deviation. The mean $(\dot{x})$, standard variation (s), and quartiles (Q) are also reported. Results indicate that the smallest error standard deviations were achieved by second order decay models. 
Table 25 Summary statistics of PPDs for the removal rate (k). Gravel Wetland (GW),

Sand Filter (SF), and Retention Pond (RP). S.S: Stormwater system. St: Statistic.

\begin{tabular}{|c|c|c|c|c|c|c|c|c|c|c|c|c|c|}
\hline \multirow{2}{*}{ S.S } & \multirow{2}{*}{ St } & \multicolumn{4}{|c|}{$n=0$} & \multicolumn{4}{|c|}{$n=1$} & \multicolumn{4}{|c|}{$n=2$} \\
\hline & & TSS & $\mathrm{Zn}$ & TPH-D & DIN & TSS & $\mathrm{Zn}$ & TPH-D & DIN & TSS & $\mathrm{Zn}$ & TPH-D & DIN \\
\hline \multirow{7}{*}{ GW } & $\dot{x}$ & 191 & 0.8 & 3.3 & 0.9 & 750 & 53 & 64 & 5.0 & 71 & 2071 & 94 & 7 \\
\hline & $\mathbf{s}$ & 30 & 0.9 & 1.5 & 0.5 & 118 & 15 & 42 & 1.7 & 40 & 562.2 & 29 & 2 \\
\hline & $Q_{90}$ & 231 & 1.8 & 5.4 & 1.6 & 892 & 74 & 133 & 7.5 & 122 & 2782 & 134 & 9 \\
\hline & $Q_{75}$ & 209 & 0.9 & 4.3 & 1.3 & 842 & 64 & 82 & 6.1 & 80 & 2541 & 109 & 9 \\
\hline & $Q_{50}$ & 194 & 0.5 & 3.2 & 0.9 & 761 & 53 & 50 & 4.8 & 59 & 2115 & 88 & 7 \\
\hline & $Q_{25}$ & 172 & 0.3 & 2.3 & 0.5 & 650 & 41 & 33 & 3.7 & 44 & 1630 & 74 & 6 \\
\hline & $Q_{10}$ & 147 & 0.1 & 1.3 & 0.2 & 597 & 32 & 22 & 2.8 & 38 & 1252 & 63 & 5 \\
\hline \multirow{7}{*}{ SF } & $\dot{x}$ & 67 & 1.5 & 2.7 & 0.7 & 92 & 89 & 37 & 1.8 & 2.3 & 1626 & 58 & 3 \\
\hline & s & 39 & 1.8 & 0.8 & 0.6 & 28 & 13 & 11 & 1.6 & 2.0 & 380.6 & 15 & 2 \\
\hline & $Q_{90}$ & 121 & 3.8 & 3.9 & 1.5 & 127 & 105 & 51 & 4.1 & 4.1 & 2133 & 79 & 6 \\
\hline & $Q_{75}$ & 92 & 1.9 & 3.3 & 1.0 & 106 & 97 & 43 & 2.5 & 2.5 & 1861 & 67 & 4 \\
\hline & $Q_{50}$ & 63 & 0.9 & 2.8 & 0.6 & 86 & 86 & 35 & 1.5 & 1.7 & 1568 & 57 & 2 \\
\hline & $Q_{25}$ & 35 & 0.4 & 2.1 & 0.2 & 73 & 80 & 29 & 0.6 & 1.3 & 1337 & 46 & 1 \\
\hline & $Q_{10}$ & 18 & 0.2 & 1.7 & 0.1 & 64 & 74 & 26 & 0.2 & 0.9 & 1181 & 40 & 0 \\
\hline \multirow{7}{*}{$\mathrm{RP}$} & $\overline{\dot{x}}$ & 47 & 1.8 & 1.2 & 0.9 & 39 & 406 & 47 & 1.0 & 1.4 & 4544 & 77 & 14 \\
\hline & $\mathbf{s}$ & 29 & 2.3 & 0.7 & 0.8 & 60 & 158 & 47 & 1.0 & 1.9 & 1417 & 38 & 17 \\
\hline & $Q_{90}$ & 86 & 5.6 & 2.1 & 1.9 & 95 & 628 & 120 & 2.2 & 3.1 & 6439 & 131 & 36 \\
\hline & $Q_{75}$ & 64 & 2.3 & 1.6 & 1.2 & 37 & 536 & 60 & 1.3 & 1.5 & 5796 & 96 & 17 \\
\hline & $Q_{50}$ & 42 & 0.7 & 1.2 & 0.7 & 17 & 407 & 26 & 0.7 & 0.8 & 4645 & 68 & 8 \\
\hline & $Q_{25}$ & 25 & 0.4 & 0.6 & 0.3 & 9 & 277 & 16 & 0.3 & 0.5 & 3395 & 48 & 3 \\
\hline & $Q_{10}$ & 13 & 0.2 & 0.2 & 0.1 & 5 & 192 & 10 & 0.1 & 0.3 & 2554 & 38 & 1 \\
\hline
\end{tabular}


Table 26 Summary statistics of PPDs for the error standard deviation $(\sigma)$. Gravel Wetland (GW), Sand Filter (SF), and Retention Pond (RP). S.S: Stormwater system. St: Statistic.

\begin{tabular}{|c|c|c|c|c|c|c|c|c|c|c|c|c|c|}
\hline \multirow{2}{*}{ S.S } & \multirow{2}{*}{ St } & \multicolumn{4}{|c|}{$\mathrm{n}=0$} & \multicolumn{4}{|c|}{$n=1$} & \multicolumn{4}{|c|}{$n=2$} \\
\hline & & TSS & $\mathrm{Zn}$ & TPH-D & DIN & TSS & $\mathrm{Zn}$ & TPH-D & DIN & TSS & $\mathrm{Zn}$ & TPH-D & DIN \\
\hline \multirow{7}{*}{ GW } & $\dot{x}$ & 39 & 0.10 & 0.49 & 0.36 & 6 & 0.019 & 0.23 & 0.32 & 6 & 0.018 & 0.20 & 0.31 \\
\hline & s & 3 & 0.11 & 0.08 & 0.03 & 0.4 & 0.002 & 0.02 & 0.02 & 0.4 & 0.001 & 0.02 & 0.02 \\
\hline & $Q_{90}$ & 42 & 0.22 & 0.58 & 0.39 & 7 & 0.021 & 0.26 & 0.35 & 6 & 0.020 & 0.22 & 0.34 \\
\hline & $\mathrm{Q}_{75}$ & 40 & 0.10 & 0.52 & 0.37 & 6 & 0.020 & 0.24 & 0.33 & 6 & 0.019 & 0.21 & 0.32 \\
\hline & $Q_{50}$ & 38 & 0.05 & 0.46 & 0.35 & 6 & 0.019 & 0.23 & 0.32 & 6 & 0.018 & 0.20 & 0.31 \\
\hline & $Q_{25}$ & 37 & 0.04 & 0.44 & 0.34 & 6 & 0.018 & 0.22 & 0.31 & 5 & 0.017 & 0.19 & 0.30 \\
\hline & $\mathbf{Q}_{10}$ & 35 & 0.04 & 0.41 & 0.33 & 6 & 0.017 & 0.21 & 0.29 & 5 & 0.017 & 0.18 & 0.29 \\
\hline \multirow{7}{*}{ SF } & $\dot{x}$ & 49 & 0.14 & 0.54 & 0.61 & 34 & 0.024 & 0.33 & 0.60 & 31 & 0.021 & 0.29 & 0.61 \\
\hline & $\mathbf{s}$ & 3 & 0.13 & 0.04 & 0.04 & 2 & 0.002 & 0.02 & 0.04 & 2 & 0.001 & 0.02 & 0.04 \\
\hline & $Q_{90}$ & 53 & 0.31 & 0.59 & 0.67 & 36 & 0.026 & 0.36 & 0.66 & 34 & 0.023 & 0.32 & 0.66 \\
\hline & $Q_{75}$ & 51 & 0.15 & 0.57 & 0.64 & 35 & 0.025 & 0.34 & 0.63 & 32 & 0.022 & 0.31 & 0.64 \\
\hline & $\mathrm{Q}_{50}$ & 49 & 0.08 & 0.54 & 0.61 & 34 & 0.024 & 0.33 & 0.60 & 31 & 0.021 & 0.29 & 0.61 \\
\hline & $\mathbf{Q}_{25}$ & 46 & 0.06 & 0.51 & 0.58 & 32 & 0.022 & 0.31 & 0.58 & 30 & 0.020 & 0.28 & 0.58 \\
\hline & $\mathbf{Q}_{10}$ & 45 & 0.06 & 0.49 & 0.56 & 31 & 0.022 & 0.29 & 0.55 & 28 & 0.019 & 0.27 & 0.56 \\
\hline \multirow{7}{*}{$\mathrm{RP}$} & $\dot{x}$ & 46 & 0.25 & 0.41 & 0.46 & 44 & 0.025 & 0.35 & 0.37 & 42 & 0.024 & 0.32 & 0.38 \\
\hline & s & 3 & 0.32 & 0.04 & 0.12 & 3 & 0.002 & 0.03 & 0.03 & 3 & 0.002 & 0.03 & 0.03 \\
\hline & $Q_{90}$ & 50 & 0.80 & 0.45 & 0.60 & 49 & 0.028 & 0.38 & 0.41 & 47 & 0.027 & 0.35 & 0.43 \\
\hline & $Q_{75}$ & 48 & 0.29 & 0.43 & 0.50 & 47 & 0.026 & 0.36 & 0.39 & 44 & 0.025 & 0.34 & 0.40 \\
\hline & $Q_{50}$ & 46 & 0.09 & 0.40 & 0.43 & 44 & 0.025 & 0.34 & 0.37 & 42 & 0.024 & 0.32 & 0.38 \\
\hline & $Q_{25}$ & 44 & 0.06 & 0.38 & 0.39 & 42 & 0.023 & 0.33 & 0.35 & 40 & 0.022 & 0.30 & 0.36 \\
\hline & $Q_{10}$ & 42 & 0.05 & 0.36 & 0.36 & 40 & 0.022 & 0.31 & 0.34 & 39 & 0.021 & 0.28 & 0.35 \\
\hline
\end{tabular}

\section{Model Validation}

A Bayesian approach was performed to validate the models. Water quality information for some storm events that were available and not included in the calibration phase was used in this step. The 05/02/2006 storm for the sand filter (Figure 32), the 07/22/2006 storm event was used for the gravel wetland (Figure 33), and the 06/01/2006 storm for the retention pond (Figure 34). The analysis was performed using a second order decay model. 
To perform the Bayesian predictive analysis, a vector of 1000 pairs of parameter values $\left(k, \sigma^{2}\right)$ was generated by using the Metropolis - Hastings output. Each removal rate was used to calculate a first estimated effluent concentration $C_{\text {out }, \text { est }}^{k}$. Afterward, a final estimated effluent concentration $C_{\text {out,est }}^{k, \sigma^{2}}$ was randomly generated from a normal distribution as follows:

$$
C_{\text {out }, \text { est }}^{k, \sigma^{2}} \sim \mathcal{N}\left(C_{\text {out }, \text { est }}^{k}, \sigma^{2}\right)
$$

Figure 32 shows predicted effluent concentrations results for the sand filter. Normally, the median is adopted as a measure of the overall behavior of the targeted variable (Beven and Binlay, 1992). In general, observed concentration values fell within the uncertainty limits. Only for DIN, one observed concentration fell outside the uncertainty limits. Concentration values below the detection limit were estimated by the model, in particular for TPH-D and Zn, where only the first sample was reported with a concentration above the detection limit. 

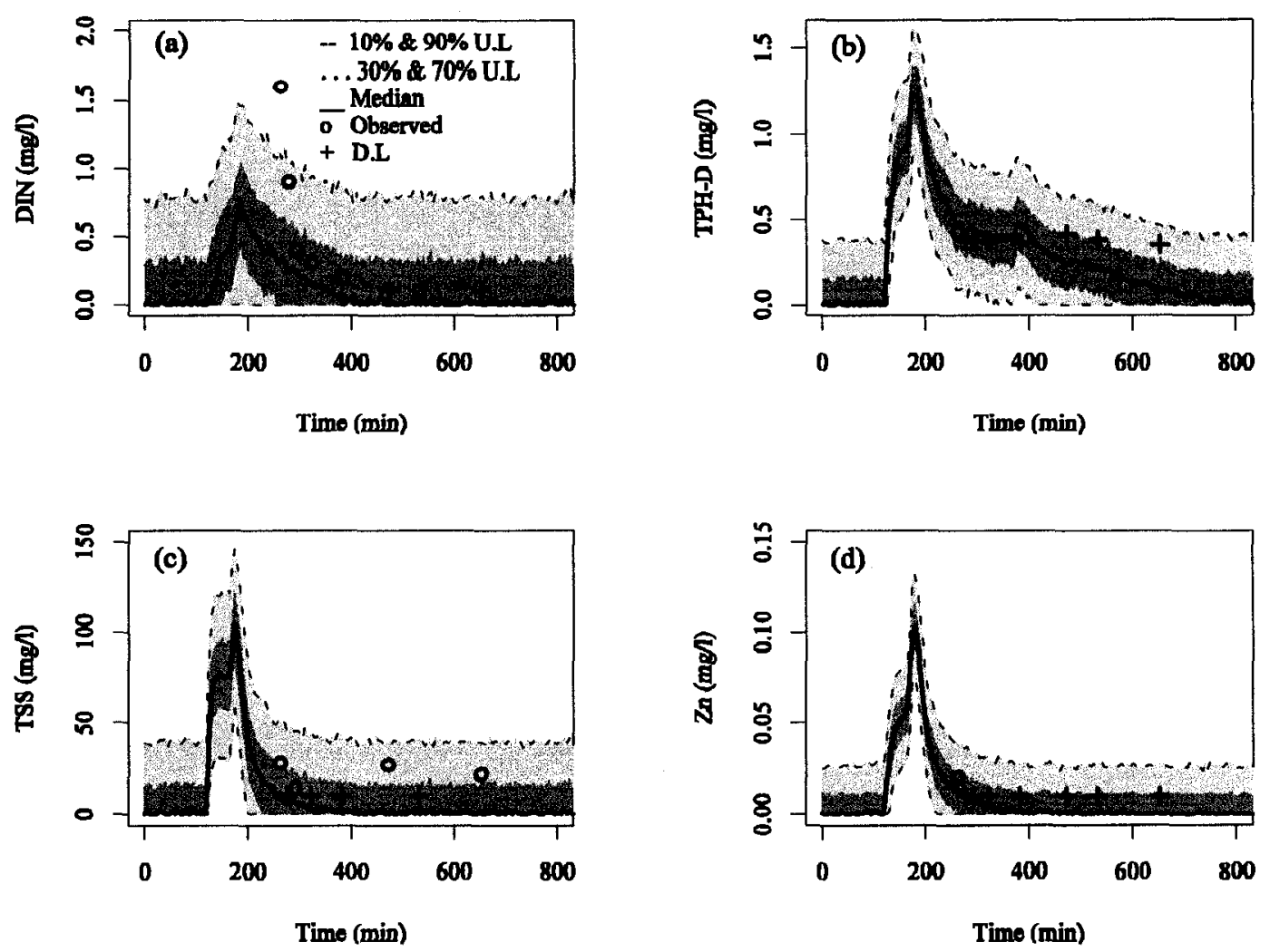

Figure 32 Monte Carlo simulations of predicted sand filter effluent pollutant concentrations for the 05/02/2006 storm event. U.L: Uncertainty limits. D.L: Detection limit. The dark shaded region indicates the $30 \%$ and $70 \%$ uncertainty limits. The light grey shaded region indicates the $10 \%$ and $90 \%$ uncertainty limits. Observed concentrations values and the reported detection limits were plotted as discrete points.

Figure 33 displays simulation results for the gravel wetland. Water quality information for 4 samples was available. Figure $33(a)$ and $33(d)$ show that for this storm, a significant range of the estimated effluent concentrations for DIN and $\mathrm{Zn}$ fell above the reported detection limit. This is particularly appreciable for DIN, since the median estimated concentration values are much higher than the detection limit. It is difficult to explain this poor performance since excellent DIN and $\mathrm{Zn}$ treatment has been reported for the gravel wetland (Roseen et al., 2006). However, an analysis of a bigger number of 
storms during the validation phase is advised. Model performance for TSS and TPH-D is acceptable considering the agreement between estimated and observed concentrations. For these contaminants, the mean estimated concentration values fell below the reported detection limit.
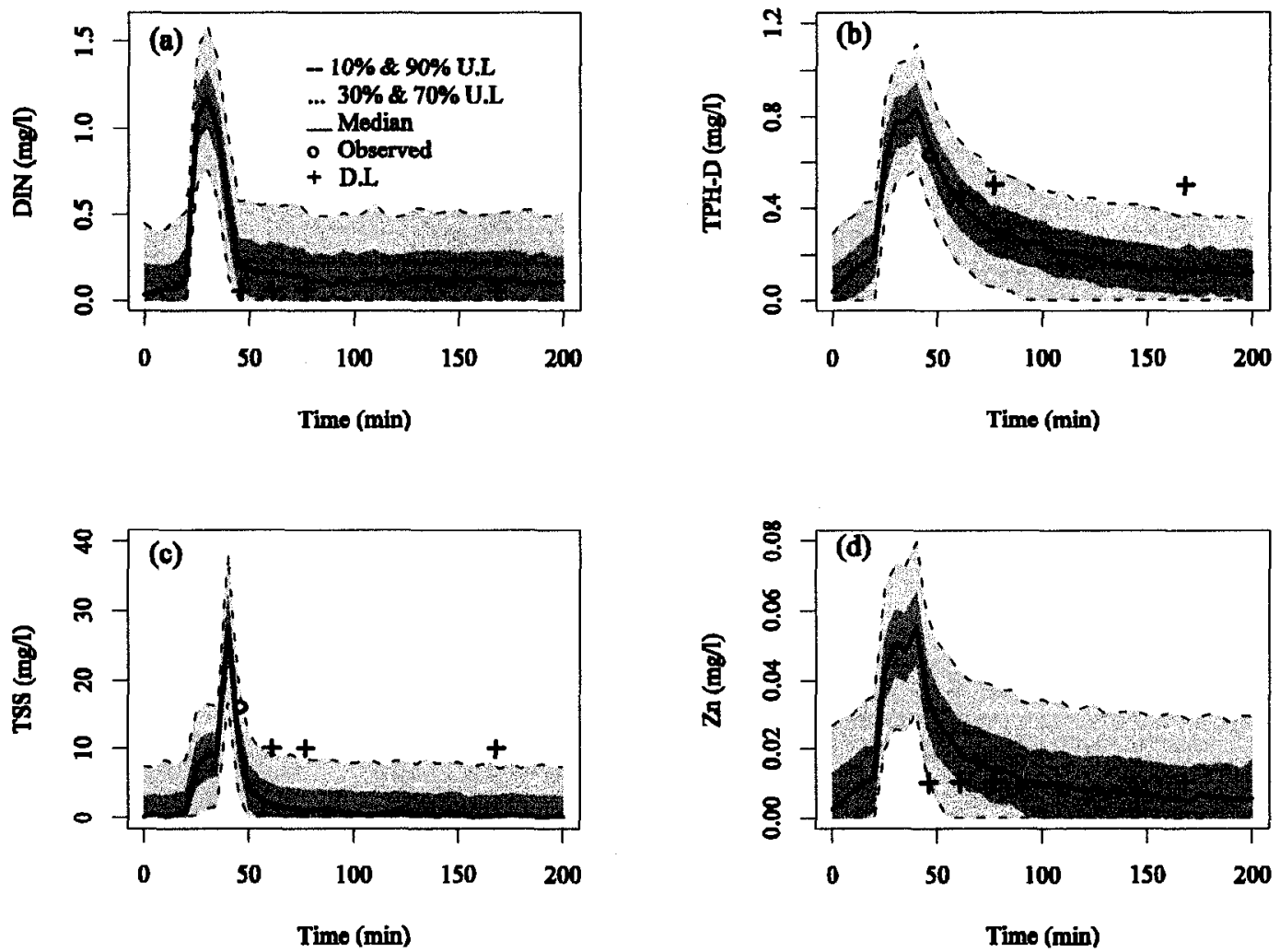

Figure 33 Monte Carlo simulations of predicted gravel wetland effluent pollutant concentrations for the 07/22/2006 storm. U.L: Uncertainty limits. D.L: Detection limit. The dark shaded region indicates the $30 \%$ and $70 \%$ uncertainty limits. The light grey shaded region indicates the $10 \%$ and $90 \%$ uncertainty limits. Observed concentrations values and the reported detection limits were plotted as discrete points.

Simulation results for the retention pond are shown in Figure 34. Observed concentration values fell within the uncertainty limits for all the contaminants. However, 
the median estimated concentrations fell below the observed concentrations for DIN, TSS, and $\mathrm{Zn}$. For TPH-D, the model predicted concentrations higher than the detection limit for samples 1 to 4 and lower values for samples 5 to 8 .
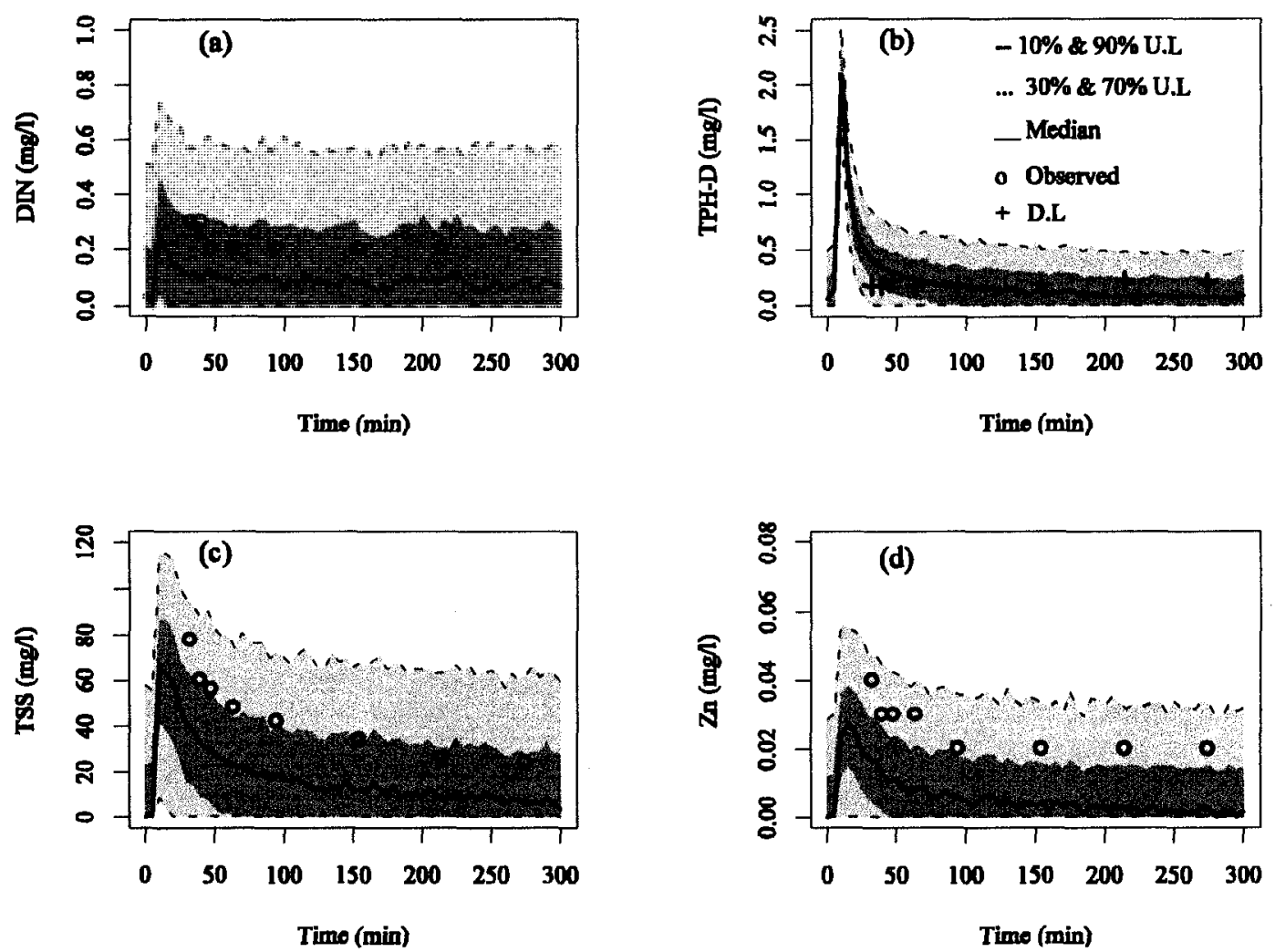

Figure 34 Monte Carlo simulations of predicted retention pond effluent pollutant concentrations for the 06/01/2006 storm. U.L: Uncertainty limits. D.L: Detection limit. The dark shaded region indicates the $30 \%$ and $70 \%$ uncertainty limits. The light grey shaded region indicates the $10 \%$ and $90 \%$ uncertainty limits. Observed concentrations values and the reported detection limits were plotted as discrete points.

Uncertainties associated with the estimated effluent pollutant concentrations can be reduced by improving the structure of the treatment model or updating the likelihood function by incorporating more field observations. Chen and Adams (2006) investigated 
a dynamic settling model in storage / treatment facilities (for example a retention pond). Parameters such as the settling velocity of the particle of concern, the settling depth, the average detention time, and the average surface area were considered in the model. Other investigations (Beven and Binley, 1992) have suggested different likelihood functions for comparing observations and predictions of the model. Arabi et al. (2007) explored a likelihood function based on the Nash-Sutcliffe efficiency criterion and recommended its application for calibration of watershed models.

\section{Conclusions}

A Bayesian approach for parameter estimation of a stormwater quality treatment model was presented in this paper. Pollutant removal was assumed to follow an exponential decay model with parameters $k$ (removal rate) and $n$ (decay order). The calibration of the model was performed for three stormwater systems: a gravel wetland, a retention pond, and a sand filter; all monitored on a side by side fashion and treating equivalent stormwater from a nearby commuter parking lot. The implicit characteristics of the method allowed for an uncertainty analysis of the estimated effluent concentrations. Water quality information consisted of influent and effluent concentrations for TSS, TPHD, DIN, and $\mathrm{Zn}$. This information was available for 23 storms collected during the 20042006 period, although not all the systems were monitored for every storm.

Zero, first, and second decay models were analyzed. Posterior probability distributions (PPDs) for the removal rate and the error variance were obtained for each system and contaminant. It was found that a second order decay model was more likely to reproduce the observed effluent concentrations. Mean removal rate values were computed from the posterior distributions. Specifically, for the gravel wetland: $k_{T S S}=59$, $k_{\mathrm{Zn}}=2115, \mathrm{k}_{\mathrm{TPH}-\mathrm{D}}=88, \mathrm{k}_{\mathrm{DIN}}=7$; for the sand filter: $\mathrm{k}_{\mathrm{TSS}}=1.7, \mathrm{k}_{\mathrm{Zn}}=1568, \mathrm{k}_{\mathrm{TPH}-\mathrm{D}}=57, \mathrm{k}_{\mathrm{DIN}}$ 
$=2$; and for the retention pond: $\mathrm{k}_{\mathrm{TSS}}=0.8, \mathrm{k}_{\mathrm{Zn}}=4645, \mathrm{k}_{\mathrm{TPH}-\mathrm{D}}=68, \mathrm{k}_{\mathrm{DIN}}=8$ (k in units of $(\mathrm{mg} /)^{-1} /$ day). A summary of basic statistics of the PPDs was provided as well (Tables 25 and 26). These PPDs can be used as prior distributions in further research. However, further research should take into account the system design characteristics.

A Bayesian predictive approach was performed to estimate effluent concentrations from the stormwater systems. For this purpose, information for one storm was not included in the calibration phase, so it could be used for validation. Most observed concentrations fell within the uncertainty limits provided by the model. As for any other model application, it is strongly recommended to explore the predictive capabilities of the model with more field data. Further research should explore a likelihood function based on the Nash-Sutcliffe efficiency criterion, which has been reported as a better measured of the model performance (Engel et al., 2007).

The conceptual formulation of the decay treatment model can be improved by incorporating new parameters that represent specific characteristics of the removal processes. In that sense, settling velocity is a parameter that needs to be considered when improving the model for a retention pond. Other factors such as vegetation are important and should be considered in further investigations.

This paper provided useful statistical information for modeling water quality treatment from stormwater systems. Model results should be interpreted carefully and used in context, especially when extensive field information is not available. Further research should look at improving the conceptualization of the exponential decay treatment model and the Bayesian statistical approach. A revision of the treatment processes will help identify other variables that may play a significant role. As for the statistical analysis, a Bayesian approach that includes both the accumulation and wash-off model and the stormwater treatment model is suggested. 


\section{REFERENCES}

Ahyerre, M., Chebbo, G., Tassin, B., and Gaume, E. (1998). Storm water quality modeling, an ambitious objective? Water Science and Technology. 37 (1): 205-213.

Alley, W.M., and Smith., P.E. (1981). Estimation of accumulation parameters for urban runoff quality modeling. Water Resources Research. 17 (6): 1657-1664.

APHA, AWWA, and WEF. (2005). Standard Methods for the examination of water and wastewater. $21^{\text {st }}$ Edition. American Public Association, Washington, D.C.

Arabi, Mazdak., Govindaraju, Rao., Hantush, Mohamed., and Engel, Bernard. (2006). Role of watershed subdivision on modeling the effectiveness of best management practices with SWAT. Journal of the American Water Resources Association. 42 (2):513-• 528.

Arabi, Mazdak., Govindaraju, Rao., and Hantush, Mohamed. (2007). A probabilistic approach for analysis of uncertainty in the evaluation of watershed management practices. Journal of Hydrology. 333: 459-471.

Avellaneda, P., Ballestero, T., Roseen, R., Houle, J. (2008). On parameter estimation of an urban stormwater runoff model. In preparation. 
Avellaneda, P., Ballestero, T., Roseen, R., and Houle, J. (2008b). Modeling urban stormwater quality treatment of a surface sand filter. In preparation.

Avellaneda, P., Ballestero, T., Roseen, R., and Houle, J. (2008c). Modeling urban stormwater quality treatment of a gravel wetland, a retention pond, and a sand filter. In preparation.

Ballestero, T., Roseen, R., Houle, J. (2005). Data Report. University of New Hampshire Stormwater Center, Durham, NH.

Barrett, M. (2005). Performance comparison of structural stormwater best management practices. Water Environmental Research. Vol 77, No 1, 78-86.

Bates, B., and Campbell, E. (2001). A Markov chain Monte Carlo scheme for parameter estimation and inference in conceptual rainfall-runoff modeling. Water Resources Research. 37(4): 937-947.

Beck, M.B. (1987). Water quality modeling: A review of the analysis of uncertainty. Water Resources Research. Vol 23, No 8, 1393-1442.

Beven, K. and Binley, A. (1992). The future of distributed models: model calibration and uncertainty prediction. Hydrological Processes. 6: 279-298.

Campos, L., Smith, S.R., Graham, N.J. (2006). Deterministic-based model of slow sand filtration. I: Model development. Journal of Environmental Engineering. Vol. 132, N0. 8, 872-886. 
Capellos, C., and Bielski, B. (1972). Kinetic systems: Methematical description of chemical kinetics in solutions. Wiley \& Sons, Inc. USA.

Charbeneau, R.J., and Barrett, M.E. (1998). Evaluation of methods for estimating stormwater pollutant loads. Water Environmental Research. 70 (7):1295-1302.

Chen, J., and Adams, B.J. (2006). A framework for urban storm water modeling and control analysis with analytical models. Water Resources Research. 42, W06419, doi:10.1029/2005WR004540.

DeCoursey, D.G. (1985). Mathematical models for nonpoint water pollution control. Journal of Soil and Water Conservation. 5: 408-413.

Edwards, D.R., Daniel, T.C., Scott, H.D., Murdoch, J.F., Habiger, M.J., and Burkes, H.M. (1996). Stream quality impacts of best management practices in a northwestern Arkansas basin. Journal of the American Water Resources Association. 32 (3):499-509.

Engel, B., Storm, D., White, M., Arnold, J., and Arabi, M. (2007). A hydrologic / water quality model application protocol. Journal of the American Water Resources Association. Vol. 43, No. 5, pp 1223-1236.

EPA. (1996). Overview of the stormwater program. Office of water. U.S. Environmental Protection Agency. Washington, D.C. EPA 833-R-96-008. 
EPA. (1999). Stormwater technology fact sheet: sand filters. U.S. Environmental Protection Agency. Washington, D.C. EPA 832-F-96-007.

EPA. (2002). Urban stormwater BMP performance monitoring - A guidance manual for meeting the national stormwater BMP database requirement. Office of Water, EPA-821B-02-001, Washington, DC.

EPA. (2006). Guidance on systematic planning using the data quality objectives process. EPA QA/G-4. Environmental Protection Agency. US.

Gaume, E., Villanueve, J.P., and Desbordes, Michel. (1998). Uncertainty assessment and analysis of the calibrated parameter values of an urban storm water quality model. Journal of Hydrology. 210: 38-50.

Gelman, A., Carlin, J.B., Stern, H.S., Rubin, D.B. (2004). Bayesian data analysis. Chapman \& Hall / CRC. Boca Raton, Florida.

Haiping, Z., and Yamada, K. (1996). Estimation for urban runoff quality modeling. Water Science and Technology. 34 (3-4): 49-54.

Hastings, W.K. (1970). Monte Carlo sampling methods using Markov chains and their applications Biometrika. 57: 97-109

Helsel, D.R., and Hirsch, R.M. (2002). Statistical methods in water resources. Technical report. USGS. USA. 
Hopgood, A.A. (2001). Intelligent systems for engineers and scientists. CRC Press. $2^{\text {nd }}$ Ed. United States.

Hong, B., Strawderman, R., Swaney, D., and Weinstein, D. (2005). Bayesian estimation of input parameters of a nitrogen cycle model applied to a forested reference watershed, Hubbard Brook Watershed Six. Water Resources Research. 41, W03007, doi:10.1029/2004WR003551.

House, M.A., Ellis, J.B., Herricks, E.E., Hvitved-Jacobsen, T., Seager, J., Lijklema, L., Aalderink, H., and Clifforde, I.T. (1993). Urban drainage - Impacts on receiving water quality. Water Science and Technology. 27 (12): 117-158.

Ice, George. (2004). History of innovative Best Management Practice development and its role in addressing water quality limited waterbodies. Journal of Environmental Engineering. 130 (6): 684-689.

Iguzquiza, P. (1998). Optimal selection of number and location of rainfall gauges for areal rainfall estimation using geostatistics and simulated annealing. Journal of Hydrology. 210 (1-4): 206-220.

Iwasaki, T. (1937). Some notes on sand filtration. J. Am. Water Works Assoc., 29(10), $1591-1602$.

Jakeman, A.J., and Hornberger, G.M. (1993). How much complexity is warranted in a rainfall-runoff model?. Water Resources Research. 29 (8): 2637-2649. 
Kadlec, R.H., and Knight, R.L. (1996). Treatment wetlands. CRC Press, Boca Raton, Florida.

Kadlec, R.H. (2000). The inadequacy of first-order treatment wetlands models. Ecological Engineering. 15: 105-119.

Kanso, A., Gromaire, M.C., Gaume, E., Tassin, B., and Chebbo, G. (2003). Bayesian approach for the calibration of models: application to an urban stormwater pollution model. Water Science and Technology. 47 (4): 77-84.

Kayhanian, M., and Stenstrom, M.K. (2005). Mass loading of first flush pollutants with treatment strategies simulations. Journal of the Transportation Research Board. No. 1904: 133-143.

Kirkpatrick, S., Gelatt, Jr., and Vecchi, M.P. (1983). Optimization by simulated annealing. Science. V 220 N 4598: 671-680.

Kottegoda, N., and Rosso, R. (1997). Statistics, probability, and reliability for civil and environmental engineers. McGraw Hill. Singapore.

Lee, J.H., and Bang, W. (2000). Characterization of urban stormwater runoff. Water Research. Vol 34, No 6, 1773-1780.

Marshall L., D. Nott, A. Sharma (2004). A comparative study of Markov chain Monte Carlo methods for conceptual rainfall-runoff modeling. Water Resources. Research. 40, W02501, doi:10.1029/2003WR002378. 
Massey, F. (1951). The Kolmogorov-Smirnov test for goodness-of-fit. Journal of the American Statistical Association. 46 (253): 68-71.

Millar, R.G. (1999). Analytical determination of pollutant wash-off parameters. Journal of Environmental Engineering. 125 (10): 989-992.

Miller, L. (1956). Table of percentage points of Kolmogorov statistics. Journal of the American Statistical Association. 51 (273): 111-121.

Minton, G. (2002). Stormwater Treatment: biological, chemical, and engineering principles. Resource Planning Associates. Washington, USA.

New York State Stormwater Management Design Manual (2001). Prepared by Center for Watershed Protection, Ellicott City, Md., for New York State, Department of Environmental Conservation, Albany, NY.

Pitt, R., Field, R., Lalor, M., and Brown, M. (1995). Urban stormwater toxic pollutants: assessment, sources, and treatability. Water Environment Research. 67, 260-275.

Reis Jr, D., and Stedinger, J. (2005). Bayesian MCMC flood frequency analysis with historical information. Journal of Hydrology. 313: 97-116.

Renard B., V. Garreta, M. Lang (2006). An application of Bayesian analysis and Markov chain Monte Carlo methods to the estimation of a regional trend in annual maxima. Water Resources Research. 42, W12422, doi:10.1029 / 2005 WR004591. 
Roseen, R.M, Ballestero, T.P, Houle, J.H, Avellaneda, P.M, Wildey, R, and Briggs, J. (2006). Storm water low-impact development, conventional structural and manufactured treatment strategies for parking lot runoff. Journal of the Transportation Research Board. 1984: $135-147$.

Rossman, L.A. (2004). Stormwater management model SWMM: user's manual version 5. Technical report. EPA. USA.

Sansalone, J., and Chad, M.C. (2004). First flush concepts for suspended and dissolved solids in small impervious watersheds. Journal of Environmental Engineering. Vol 130, No. $11,1301-1314$.

Sartor, J.D., Boyd, G.B., and Agardy, Franklin. (1974). Water pollution aspects of street surface contaminants. Journal of Water Pollution Control Federation. 46 (3): 458 - 467.

Seidou O., T. B. M. J. Ouarda, M. Barbet, P. Bruneau, B. Bobée (2006). A parametric Bayesian combination of local and regional information in flood frequency analysis. Water Resources Research. 42, W11408, doi:10.1029 / 2005 WR004397.

Shaheen, D.G. (1975). Contributions of urban roadway to water pollution. U.S. Environmental Protection Agency. Off. Res. Dev. Rep., EPA-600/2-75-004.

Steinberg, L., Reckhow, K., and Wolpert, Robert. (1996). Bayesian model for fate and transport of polychlorinated biphenyl in upper Hudson River. Journal of Environmental Engineering. 122 (5): 341-349. 
Tsihrintzis, V.A., and Hamid, R. (1997). Modeling and management of urban stormwater runoff quality: a review. Water Resources Management. 11: 137-164.

Urbonas, B.R. (1995). Recommended parameters to report with BMP monitoring data. Journal of Water Resources Planning and Management. 121 (1): 23-34.

Walker, J.F. (1994). Statistical techniques for assessing water-quality effects of BMPs. Journal of Irrigation and Drainage Engineering. Vol 120, No 2, 334-347.

Wang, G., Chen, S., Barber, M.E., and Yonge, D.R. (2004). Modeling flow and pollutant removal of wet detention pond treating stormwater runoff. Journal of Environmental Engineering. 130 (11): 1315-1321.

Wong, T.H.F, Duncan, H.P., Fletcher, T.D. and Jenkins, G.A. (2006). Modelling urban stormwater treatment - a unified approach. Ecological Engineering. 27 (1): 58-70.

Zoppou, C. (2001). Review of urban storm water models. Environmental Modelling \& Software. 16: 195-231. 


\section{APPENDIX A: FUNDAMENTAL EQUATIONS}

\section{Zero-order decay model}

For a zero-order decay model $(n=0)$, the governing differential equation (Equation (8)) can be written as follows:

$$
\frac{d C_{\text {out }}}{d t}=\frac{C_{\text {in }} Q_{\text {in }}-C_{\text {out }} Q_{\text {out }}}{S}-\frac{C_{\text {out }}}{S} \frac{d S}{d t}-k
$$

Direct integration of this equation gives the following analytical solution:

$$
\begin{aligned}
& C_{\text {out }}=\frac{A}{B}+\left(C_{0}-\frac{A}{B}\right) e^{-B \Delta} \\
& A=\frac{C_{\text {in }} Q_{\text {in }}}{S}-k \\
& B=\frac{Q_{\text {out }}+\frac{d S}{d t}}{S}
\end{aligned}
$$

where $C_{\text {out }}$ is the effluent concentration $(\mathrm{mg} / \mathrm{l}) ; \mathrm{C}_{\text {in }}$ is the influent concentration $(\mathrm{mg} / \mathrm{l}) ; \mathrm{C}_{0}$ is the initial concentration within the system $(\mathrm{mg} / \mathrm{l}) ; Q_{\text {in }}$ is the influent flow rate $\left(\mathrm{m}^{3} /\right.$ day); $Q_{\text {out }}$ is the effluent flow rate $\left(\mathrm{m}^{3} /\right.$ day); $S$ is the storage within the system $\left(\mathrm{m}^{3}\right)$; $d S / d t$ is the change in storage $\left(\mathrm{m}^{3} /\right.$ day); $\Delta t$ is the time interval $(\mathrm{min})$; and $\mathrm{k}$ is the removal rate $\left((\mathrm{mg} / \mathrm{l})^{-\mathrm{n}+1} / \mathrm{day}\right)$. 


\section{First-order decay model}

For a first-order decay model $(n=1)$, the governing equation (Equation (8)) can be written as follows:

$$
\frac{d C_{\text {out }}}{d t}=\frac{C_{\text {in }} Q_{\text {in }}-C_{\text {out }} Q_{\text {out }}}{S}-\frac{C_{\text {out }}}{S} \frac{d S}{d t}-k C_{\text {out }}
$$

An analytical solution can be found by direct integration:

$$
\begin{aligned}
& C_{\text {out }}=\frac{D}{E}+\left(C_{0}-\frac{D}{E}\right) e^{-E \Delta t} \\
& D=\frac{C_{\text {in }} Q_{\text {in }}}{S} \\
& E=\frac{Q_{\text {out }}+\frac{d S}{d t}}{S}+k
\end{aligned}
$$

\section{Second-order decay model}

For a second-order decay model $(n=2)$, the governing equation (Equation (8)) can be written as follows:

$$
\frac{d C_{\text {out }}}{d t}=\frac{C_{\text {in }} Q_{\text {in }}-C_{\text {out }} Q_{\text {out }}}{S}-\frac{C_{\text {out }}}{S} \frac{d S}{d t}-k C_{\text {out }}^{2}
$$

This is a form of the Riccati equation and has the following analytical solution: 


$$
\begin{aligned}
& C_{\text {out }}=\frac{1}{C_{0}-\lambda}+\frac{k_{3}}{k_{2}+2 \lambda k_{3}} \\
& k_{1}=\frac{C_{\text {in }} Q_{\text {in }}}{S} \\
& k_{2}=\frac{Q_{\text {out }}+\frac{d S}{d t}}{S} \\
& k_{3}=k \\
& \lambda=\frac{-k_{2}+\sqrt{k_{2}^{2}+4 k_{3} k_{1}}}{2 k_{3}}
\end{aligned}
$$

